\title{
Characterisations of function spaces of generalized smoothness
}

Farkas, Walter ; Leopold, H G

DOI: https://doi.org/10.1007/s10231-004-0110-z

Posted at the Zurich Open Repository and Archive, University of Zurich ZORA URL: https://doi.org/10.5167/uzh-99904

Journal Article

Published Version

Originally published at:

Farkas, Walter; Leopold, H G (2006). Characterisations of function spaces of generalized smoothness. Annali di Matematica Pura ed Applicata, 185:1-62.

DOI: https://doi.org/10.1007/s10231-004-0110-z 
Walter Farkas · Hans-Gerd Leopold ${ }^{\star}$

\title{
Characterisations of function spaces of generalised smoothness
}

Received: March 10, 2002; in final form: March 13, 2003

Published online: June 22, 2004 - (c) Springer-Verlag 2004

\begin{abstract}
We investigate function spaces of generalised smoothness of Besov and TriebelLizorkin type. Equivalent quasi-norms in terms of maximal functions and local means are given. An atomic decomposition theorem for this type of spaces is proved.
\end{abstract}

Mathematics Subject Classification (2000). 46E35

Key words. function space - maximal function - local means - atomic decompositions

\section{Introduction}

Function spaces of generalised smoothness have been introduced and considered by several authors, in particular since the middle of the seventies up to the end of the eighties, with different starting points and in different contexts.

The Steklov Mathematical Institute in Moscow was the starting point for many contributions to the topic. M.L. Goldman and G.A. Kalyabin developed independently an approach via the approximation by series of entire analytic functions and coverings, see for example [Go79], [Go80], [Ka77a] and [Ka80]. Another approach is due to M.L. Goldman, see [Go84a], who gave a systematic treatment based on differences and moduli of the continuity of those type of spaces. His setting has P.L. Ul'yanov (1968) and A.S. Dzhafarov (1965) as forerunners.

In both cases mentioned above, the spaces consist of functions belonging to $L_{p}$ with additional smoothness properties.

Many remarkable and final results were obtained, for example results concerning embeddings in different kinds of spaces of smoothness level zero, equivalent norms, trace theorems and estimates of capacities. The survey [KaLi87], the supplement in [Tr86], or [KuNi88, Chap. 5 §4] cover, in particular, the literature up to the end of the eighties in this direction.

In our work we will take up some basic ideas from the above settings but now from the standpoint of a Fourier analytic characterisation. This allows us to obtain

W. Farkas: University of Zurich, Swiss Banking Institute, Plattenstrasse 14, CH-8032 Zurich, Switzerland, e-mail: farkas@math. ethz .ch

H.-G. Leopold: Friedrich-Schiller-Universität Jena, Mathematisches Institut, Ernst-AbbePlatz 1-4, D-07740 Jena, Germany, e-mail: leopold@minet.uni-jena. de

* Partly supported by DFG-contract Tr374/4 
the description of the full scale of spaces, including spaces of negative smoothness and duality results.

Further, spaces of generalised smoothness defined on ideal spaces $E$ as basic spaces, instead of $L_{p}$, were considered in [Go86], [Go92], [Ne88], and in [Ne89].

Moreover, at the end of the eighties C. Merucci, see [Me83], F. Cobos and D.L. Fernandez, see [CoFe86], investigated some classes of function spaces of generalised smoothness. More precisely they characterised interpolation spaces between $L_{p}$ and $W_{p}^{k}$ which were obtained with respect to a generalised real interpolation method.

We have noticed an increasing interest in spaces of generalised smoothness in the last years. First of all, this interest is in connection with embeddings, limiting embeddings and entropy numbers. We mention here the papers [Le98], [EGO97], [EdHa99], [OpTr00] where such problems were considered. Furthermore, in [Bu99] function spaces of generalised smoothness are investigated in order to characterise properties of bounded extension operators from $W_{p}^{l}(\Omega)$ into function spaces on $\mathbb{R}^{n}$ for arbitrary bounded open domains $\Omega$ with $\partial \Omega \in \operatorname{Lip} \gamma$, $0<\gamma \leqslant 1$.

Additionally, in connection with generalised $d$-sets and $h$-sets (special fractals) those spaces appeared in a natural way in [EdTr98], [EdTr99], [Mo99], [Mo01], and in [Br02].

We would like to point out that function spaces of a variable and generalised order of smoothness play a key role in other mathematical fields such as probability theory and the theory of stochastic processes.

At least since the publication of M. Fukushima's work, see [Fu71], on Dirichlet forms and Markov processes, the (functional) analytic approach to stochastic processes turned into the centre of probabilists interest. More precisely, the point of view based on the relation of Fourier analysis and Markov processes, a subject which was first taken up by P. Lévy and by S. Bochner when discussing stochastically continuous processes with stationary and independent increments, is at present an area of intensive development, see the books of N. Jacob [Ja96], [Ja01], and the references therein.

In the problem of construction and investigation of Lévy and special Markov processes, function spaces of generalised smoothness of Bessel potential type appear in a natural way.

The key observation is that every Lévy process $\left(Y_{t}\right)_{t \geqslant 0}$ with state space $\mathbb{R}^{n}$ and related to a translation invariant symmetric Dirichlet form is completely determined by one and only one function $\psi: \mathbb{R}^{n} \rightarrow \mathbb{C}$, which is defined by the relation

$$
E\left(e^{i Y_{t} \cdot \xi}\right)=e^{-t \psi(\xi)} .
$$

The function $\psi$, called the characteristic exponent of $\left(Y_{t}\right)_{t \geqslant 0}$, is a continuous negative definite function and contains all the information about $\left(Y_{t}\right)_{t \geqslant 0}$. By the exact characterisation of the domain of definition of the related semigroup one can get information about the process.

Furthermore, as a matter of fact every reasonable Feller process with state space $\mathbb{R}^{n}$ is characterised by a family (parametrised by $\mathbb{R}^{n}$ ) of continuous negative 
definite functions. More precisely, following [Ja98], see also [Sc98b], for the Feller process $\left(\left(X_{t}\right)_{t \geqslant 0}, P^{x}\right)_{x \in \mathbb{R}^{n}}$ one finds that

$$
-q(x, \xi)=\lim _{t \downarrow 0} \frac{E^{x}\left(e^{i\left(X_{t}-x\right) \cdot \xi}\right)-1}{t}
$$

is the symbol of the generator of the semigroup

$$
T_{t} u(x)=E^{x}\left(u\left(X_{t}\right)\right)
$$

associated with $\left(\left(X_{t}\right)_{t \geqslant 0}, P^{x}\right)_{x \in \mathbb{R}^{n}}$, i.e. on $C_{0}^{\infty}\left(\mathbb{R}^{n}\right)$ we have

$$
A u(x)=-q(x, D) u(x)=-(2 \pi)^{-n / 2} \int_{\mathbb{R}^{n}} e^{i x \cdot \xi} q(x, \xi) \widehat{u}(\xi) d \xi .
$$

Moreover, $\xi \mapsto q(x, \xi)$ is for each $x \in \mathbb{R}^{n}$ a continuous negative definite function. This result complements a theorem of $\mathrm{Ph}$. Courrège, see [Co66], which states that on $C_{0}^{\infty}\left(\mathbb{R}^{n}\right)$ the generator of a Feller semigroup necessarily has the structure (1.1).

Now, assuming for example that $q(x, \xi) \sim \psi(\xi)$, where $\psi: \mathbb{R}^{n} \rightarrow \mathbb{R}$ is a fixed continuous negative definite function (independent of $x$ ), one should expect that the operator $q(x, D)$ behaves up to a perturbation like $\psi(D)$. Hence the scales of spaces associated with $\psi$ should play for $q(x, D)$ the same role as Sobolev or Besov and Triebel-Lizorkin spaces do for elliptic operators in the classical situation, i.e. for operators with symbol $q(x, \xi) \sim|\xi|^{2 m}$.

This topic was discussed in [FJS01a] and [FJS01b] where the role played by some generalised Bessel potential spaces, which are domains of definition for $L_{p}$-generators of sub-Markovian semigroups, is pointed out. In particular the obtaining of embedding theorems for those spaces was a central point in that investigation.

The aim of this paper is twofold. The first is to give a unified approach on function spaces of generalised smoothness and the second one is to characterise these spaces in terms of new tools such as local means and atoms.

Our approach has as background the Fourier-analytic characterisation of function spaces based on a suitable resolution of unity on the Fourier side and a suitable weighted summation of the resulting parts.

Any temperate distribution $f \in \mathcal{f}^{\prime}\left(\mathbb{R}^{n}\right)$ is decomposed in a sum of entire analytic functions $\left(\varphi_{j} \widehat{f}\right)^{\vee}$. This decomposition in the Fourier-image is in the classical case usually related to the symbol of the Laplacian and to the sequence $2^{j}$. Then this sequence of entire analytic functions $\left(\varphi_{j} \widehat{f}\right)^{\vee}$ is considered in $L_{p}$ and afterwards in a weighted $l_{q}$ space with weight sequence $2^{s j}$ in the case of $B_{p, q}^{s}\left(\mathbb{R}^{n}\right)$, and vice-versa for $F_{p, q}^{s}\left(\mathbb{R}^{n}\right)$. These two scales $B_{p, q}^{s}\left(\mathbb{R}^{n}\right)$ and $F_{p, q}^{s}\left(\mathbb{R}^{n}\right)$ contain, as special cases, many well-known spaces such as Hölder-Zygmund spaces, Sobolev spaces, fractional Sobolev spaces, Besov spaces, inhomogeneous Hardy spaces and spaces of BMO-type.

To extend the classical construction to the case of generalised smoothness we replace the sequences $2^{j}$ and $2^{s j}$ by two sequences $N$ and $\sigma$. The first is strongly 
increasing and determines the decomposition on the Fourier side. The second one is the weight sequence for $l_{q}$ and is, together with its inverse, of bounded growth.

We show that such a construction is suitable and covers many classes of function spaces of generalised smoothness known so far in the literature. Furthermore we give a comprehensive study of those spaces including Littlewood-Paley theorems, existence of a lift operator and duality. A main point is also an equivalent characterisation of some generalised Bessel potential spaces - introduced in [FJS01a] and [FJS01b] in connection with the problem of constructing Markov processes in our context as function spaces of generalised smoothness.

In the eighties and nineties new far-reaching tools for classical spaces $B_{p, q}^{s}\left(\mathbb{R}^{n}\right)$ and $F_{p, q}^{s}\left(\mathbb{R}^{n}\right)$ have been developed. The key words are maximal functions, local means, atomic and, most recently, quarkonial decompositions.

First, under some mild restrictions on the sequence $N$ determining the decomposition on the Fourier side, we prove a general characterisation of these spaces in terms of maximal functions and local means, which essentially generalises the characterisation from [BPT96] and [BPT97] of H.-Q. Bui, M. Paluszyński, and M. Taibleson (which complemented some earlier results of J. Peetre, see [Pe75], and H. Triebel, see [Tr88] and [Tr92]). This result (see the precise formulation in Theorem 4.3.4) is of independent interest but it played the key role in proving the central result of this paper, the atomic decomposition theorem.

Entire analytic functions may be considered as building blocks for the spaces $B_{p, q}^{s}\left(\mathbb{R}^{n}\right)$ and $F_{p, q}^{s}\left(\mathbb{R}^{n}\right)$ in the sense described above or in the sense of approximation theory.

However there is a well known other type of decomposition in simple building blocks, the so-called atoms. They have a history of some twenty years and in $[\operatorname{Tr} 92$, Sect. 1.9] a historical report was given on this topic; we do not repeat it here. We only want to mention that the (smooth) atoms in $B_{p q}^{s}\left(\mathbb{R}^{n}\right)$ and $F_{p q}^{s}\left(\mathbb{R}^{n}\right)$ spaces as they were defined by M. Frazier and B. Jawerth in [FrJa85], [FrJa90] (cf. also [FJW91]), proved to be a powerful tool in the theory of function spaces. We also wish to emphasise that there exist many other types of atomic decompositions in such spaces but we will not discuss this point here. More information about this subject is given in [FrJa90], [Tr92] and [AdHe96] where one can find many modifications and applications as well as comprehensive references extending the subject.

We conclude our work by obtaining a decomposition theorem which extends the atomic decomposition theorem of M. Frazier and B. Jawerth, see [FrJa85] and [FrJa90], to the function spaces $B_{p, q}^{\sigma, N}\left(\mathbb{R}^{n}\right)$ and $F_{p, q}^{\sigma, N}\left(\mathbb{R}^{n}\right)$.

Consequently, the study of function spaces can be done with the help of some sequence spaces in an analogous way as it is done in the classical (isotropic) case in the above cited works of M. Frazier, B. Jawerth and H. Triebel.

In a forthcoming paper we will use the atomic decomposition theorem in the study of mapping properties for pseudo-differential operators on some function spaces of generalised smoothness. In particular this will allow us to discuss conditions under which pseudo-differential operators are generators of $L_{p}$-subMarkovian semigroups. 
Briefly here is the organisation and contents of this paper.

We tried to make our exposition as self-contained as possible so that Section 2 has a preparatory character. We set up notation and introduce the sequences determining the generalised smoothness.

The first sequence is a so-called strongly increasing sequence $N=\left(N_{j}\right)_{j \in \mathbb{N}_{0}}$ (an almost increasing sequence such that, additionally, there exists a natural number $\kappa_{0}$ with $2 N_{j} \leqslant N_{k}$ for all $j$ and all $k$ with $j+\kappa_{0} \leqslant k$, see the precise formulation in Definition 2.2.1) which generalises the sequence $\left(2^{j}\right)_{j \in \mathbb{N}_{0}}$ and induces a decomposition in $\mathbb{R}^{n}$ in the sets $\Omega_{j}^{N}=\left\{\xi \in \mathbb{R}^{n}:|\xi| \leqslant N_{j+\kappa_{0}}\right\}$ for $j=0,1, \ldots, \kappa_{0}-1$ and $\Omega_{j}^{N}=\left\{\xi \in \mathbb{R}^{n}: N_{j-\kappa_{0}} \leqslant|\xi| \leqslant N_{j+\kappa_{0}}\right\}$ for $j \geqslant \kappa_{0}$. To this decomposition of $\mathbb{R}^{n}$ there is associated a family $\left(\varphi_{j}^{N}\right)_{j \in \mathbb{N}_{0}}$ of compactly supported smooth functions which extends the classical partition of unity. We then have a decomposition of any tempered distribution $f$ into a series of entire analytic functions $f_{j}=\left(\varphi_{j}^{N} \widehat{f}\right)^{\vee}$ like in the classical case.

Secondly, we will consider a so-called admissible sequence $\sigma=\left(\sigma_{j}\right)_{j \in \mathbb{N}_{0}}$ (i.e. it satisfies $d_{0} \sigma_{j} \leqslant \sigma_{j+1} \leqslant d_{1} \sigma_{j}$ for any $j \in \mathbb{N}_{0}$ ) which generalises the sequence $\left(2^{j s}\right)_{j \in \mathbb{N}_{0}}$ and which is a smoothness weight for the different functions $f_{j}$. We want to point out that an admissible sequence $\sigma$ is considerably more general than $\left(2^{j s}\right)_{j \in \mathbb{N}_{0}}$ or than $\left(2^{j s} \Psi\left(2^{-j}\right)\right)_{j \in \mathbb{N}_{0}}$ (for monotone functions $\Psi$ on $(0,1]$ with $\left.\Psi\left(2^{-j}\right) \sim \Psi\left(2^{-2 j}\right)\right)$, see Example 2.2.7.

In Section 3, for given sequences $N$ and $\sigma$ and for $1<p<\infty, 1 \leqslant q \leqslant \infty$, Besov, respectively Triebel-Lizorkin, spaces of generalised smoothness are defined as the collection of all tempered distributions $f$ such that $\left\|f \mid B_{p, q}^{\sigma, N}\right\|=$ $\left\|\sigma_{j}\left(\varphi_{j}^{N} \widehat{f}\right)^{\vee} \mid l_{q}\left(L_{p}\right)\right\|$, respectively $\left\|f\left|F_{p, q}^{\sigma, N}\|=\| \sigma_{j}\left(\varphi_{j}^{N} \widehat{f}\right)^{\vee}\right| L_{p}\left(l_{q}\right)\right\|$, is finite, see Definition 3.1.2.

To show that the definition of the spaces is consistent, one has to use the classical Fourier-multiplier theorem of the Michlin-Hörmander type (for convenience we recall it in Proposition 3.1.1). It is easy to show that standard properties in the classical situation, such as the density of test functions $\&$ (for appropriate values of the parameters) are still true.

Then we prove a theorem of Littlewood-Paley type: $F_{p, 2}^{\sigma^{0}, N}=L_{p}$ for any strongly increasing sequence $N$ (here $\sigma^{0}$ denotes the sequence with all terms equal to 1 ), we prove embeddings on the level of zero-smoothness, we show the existence of a lift operator between spaces $B_{p, q}^{\sigma, N}$ and $B_{p, q}^{\beta, N}$ (and also for $F$-spaces) and finally we prove a duality result.

In particular in Subsection 3.1.2 we study some special classes of function spaces of generalised smoothness, those in which the strongly increasing sequence $N=\left(N_{j}\right)_{j \in \mathbb{N}_{0}}$ is obtained from a smooth, given, so-called admissible function (see Definition 3.1.11) in a canonical way. In particular the considerations in this subsection allow us to treat some Bessel potential spaces introduced in [FJS01a] and [FJS01b] (which appear in the context of Markov processes).

To extend the definition of the spaces of generalised smoothness to $p=\infty$, $p=1$ and $0<p<1$, an additional assumption on the sequence $N$ is necessary, namely that sequence $N$ has to be not only strongly increasing but also of bounded 
growth. The reason is that we have to use in these cases another Fourier-multiplier theorem than before. A brief discussion is contained in Subsection 3.2.

Subsection 3.3 illustrates how our approach covers many classes of function spaces of generalised smoothness known up to now in the literature.

Simultaneously, due to the flexibility of the admissible sequence $\sigma$, this covering is a strict one.

Finally, Section 4 contains the main results of this work. Under the assumption that the sequence $N=\left(N_{j}\right)_{j \in \mathbb{N}_{0}}$ satisfies $\lambda_{0} N_{j} \leqslant N_{j+1} \leqslant \lambda_{1} N_{j+1}$ for any $j \in \mathbb{N}_{0}$, with some constants $1<\lambda_{0} \leqslant \lambda_{1}$, we prove the characterisation with local means and the atomic decomposition theorem.

The theorem on local means (Theorem 4.3.4) is highly technical and its proof required (compared with the classical situation) new techniques and ideas. We summarised the basic ideas of the proof in Subsection 4.3.4.

Roughly speaking, the atomic decomposition theorem states that, for any $g \in B_{p, q}^{\sigma, N}\left(\mathbb{R}^{n}\right)$, it is possible to find a decomposition (convergence in $\delta^{\prime}\left(\mathbb{R}^{n}\right)$ )

$$
g=\sum_{\nu=0}^{\infty} \sum_{m \in \mathbb{Z}^{n}} \lambda_{v m} \rho_{v m}
$$

where $\rho_{\nu m}$ are the $N$-atoms and $\lambda=\left\{\lambda_{v m}: \nu \in \mathbb{N}_{0}, m \in \mathbb{Z}^{n}\right\}$ belongs to an appropriate sequence space $b_{p, q}$, such that

$$
\left\|g\left|B_{p, q}^{\sigma, N}\left(\mathbb{R}^{n}\right)\|\sim \inf \| \lambda\right| b_{p, q}\right\|,
$$

where the infimum is taken over all admissible representations of $g$ and

$$
\left\|\lambda \mid b_{p, q}\right\|=\left(\sum_{\nu=0}^{\infty}\left(\sum_{m \in \mathbb{Z}^{n}}\left|\lambda_{v m}\right|^{p}\right)^{q / p}\right)^{1 / q}
$$

(with the usual modification if $p=\infty$ and/or $q=\infty$ ), and a corresponding assertion for $F_{p, q}^{\sigma, N}\left(\mathbb{R}^{n}\right)$ spaces. The precise formulation is given in Theorem 4.4.3.

Finally we would like to mention that it is to be expected that our main results have an anisotropic counterpart but, due to the technical complications, no attempt in this direction has been made here.

Acknowledgements. It is a pleasure to give our warm thanks to Professor Niels Jacob for fruitful discussions and encouragement.

We also thank Michele Bricchi for his comments on a preliminary version ([FaLe01]) of this paper.

\section{Preliminaries}

\subsection{Notation}

Let $\mathbb{N}$ be the collection of all natural numbers and $\mathbb{N}_{0}=\mathbb{N} \cup\{0\}$. Let $\mathbb{R}^{n}$ be Euclidean $n$-space, where $n \in \mathbb{N}$; as usual $\mathbb{R}=\mathbb{R}^{1}$. For $x \in \mathbb{R}^{n}$ let $\langle x\rangle=\left(1+|x|^{2}\right)^{1 / 2}$. 
If $\alpha=\left(\alpha_{1}, \ldots, \alpha_{n}\right) \in \mathbb{N}_{0}^{n}$ is a multi-index its length is $|\alpha|=\alpha_{1}+\ldots+\alpha_{n}$, the derivatives $D^{\alpha}$ have the usual meaning and if $x=\left(x_{1}, \ldots, x_{n}\right) \in \mathbb{R}^{n}$ then $x^{\alpha}=x_{1}^{\alpha_{1}} \cdots x_{n}^{\alpha_{n}}$.

Let $\&\left(\mathbb{R}^{n}\right)$ be the Schwartz space of all complex-valued rapidly decreasing infinitely differentiable functions on $\mathbb{R}^{n}$ equipped with the usual topology. By $\delta^{\prime}\left(\mathbb{R}^{n}\right)$ we denote its topological dual, the space of all tempered distributions on $\mathbb{R}^{n}$. If $\varphi \in \delta\left(\mathbb{R}^{n}\right)$ then

$$
\widehat{\varphi}(\xi)=\mathcal{F} \varphi(\xi)=(2 \pi)^{-\frac{n}{2}} \int_{\mathbb{R}^{n}} e^{-i x \xi} \varphi(x) d x
$$

and $\check{\varphi}=\mathcal{F}^{-1} \varphi$ are, respectively, the Fourier and inverse Fourier transform of $\varphi$. One extends $\mathcal{F}$ and $\mathcal{F}^{-1}$ in the usual way from $\delta\left(\mathbb{R}^{n}\right)$ to $\wp^{\prime}\left(\mathbb{R}^{n}\right)$. For $\varphi \in \delta\left(\mathbb{R}^{n}\right)$ and $f \in \mathcal{S}^{\prime}\left(\mathbb{R}^{n}\right)$ we will use the notation $\varphi(D) f(x)=\left[\mathcal{F}^{-1}(\varphi \mathcal{F} f)\right](x)$, where this is the extension of

$$
\varphi(D) \psi(x)=(2 \pi)^{-n} \int_{\mathbb{R}^{n}} \int_{\mathbb{R}^{n}} e^{i(x-y) \xi} \varphi(\xi) \psi(y) d y d \xi, \quad \psi \in \delta\left(\mathbb{R}^{n}\right)
$$

to elements $f \in \mathcal{S}^{\prime}\left(\mathbb{R}^{n}\right)$.

Furthermore, $L_{p}\left(\mathbb{R}^{n}\right)$ with $0<p \leqslant \infty$, is the standard quasi-Banach space with respect to the Lebesgue measure, quasi-normed by

$$
\left\|f \mid L_{p}\left(\mathbb{R}^{n}\right)\right\|=\left(\int_{\mathbb{R}^{n}}|f(x)|^{p} d x\right)^{1 / p},
$$

with the obvious modification if $p=\infty$.

We adopt here and in the sequel the following convention: if there is no danger of confusion we omit $\mathbb{R}^{n}$ in $\&\left(\mathbb{R}^{n}\right)$ and in the other spaces below.

Let $0<q \leqslant \infty$, then $l_{q}$ is the set of all sequences $\left(a_{k}\right)_{k \in \mathbb{N}_{0}}$ of complex numbers such that

$$
\left\|\left(a_{k}\right)_{k \in \mathbb{N}_{0}} \mid l_{q}\right\|=\left(\sum_{k=0}^{\infty}\left|a_{k}\right|^{q}\right)^{1 / q}<\infty
$$

with the obvious modification if $q=\infty$.

Let $0<p \leqslant \infty$ and $0<q \leqslant \infty$. If $\left(f_{k}\right)_{k \in \mathbb{N}_{0}}$ is a sequence of complex-valued Lebesgue measurable functions on $\mathbb{R}^{n}$, then

$$
\left\|\left(f_{k}\right)_{k \in \mathbb{N}_{0}} \mid l_{q}\left(L_{p}\right)\right\|=\left(\sum_{k=0}^{\infty}\left(\int_{\mathbb{R}^{n}}\left|f_{k}(x)\right|^{p} d x\right)^{q / p}\right)^{1 / q}
$$

and

$$
\left\|\left(f_{k}\right)_{k \in \mathbb{N}_{0}} \mid L_{p}\left(l_{q}\right)\right\|=\left(\int_{\mathbb{R}^{n}}\left(\sum_{k=0}^{\infty}\left|f_{k}(x)\right|^{q}\right)^{p / q} d x\right)^{1 / p},
$$

again with obvious modifications if $p=\infty$ and/or $q=\infty$.

The equivalence $a_{k} \sim b_{k}$ or $\varphi(x) \sim \psi(x)$ means that there are two positive constants $c_{1}$ and $c_{2}$ such that $c_{1} a_{k} \leqslant b_{k} \leqslant c_{2} a_{k}$ or $c_{1} \varphi(x) \leqslant \psi(x) \leqslant c_{2} \varphi(x)$, for all admissible values of the discrete variable $k$ or of the continuous variable $x$.

All unimportant positive constants are denoted with $c$, occasionally with additional subscripts within the same formulae. 


\subsection{Sequences}

Definition 2.2.1. A sequence $\gamma=\left(\gamma_{j}\right)_{j \in \mathbb{N}_{0}}$ of positive real numbers is called:

(i) almost increasing if there is a positive constant $d_{0}$ such that

$$
d_{0} \gamma_{j} \leqslant \gamma_{k} \text { for all } j \text { and } k \text { with } 0 \leqslant j \leqslant k \text {; }
$$

(ii) strongly increasing if it is almost increasing and, in addition, there is a natural number $\kappa_{0}$ such that

$$
2 \gamma_{j} \leqslant \gamma_{k} \text { forall } j \text { and } k \text { with } j+\kappa_{0} \leqslant k
$$

(iii) of bounded growth if there are a positive constant $d_{1}$ and a number $J_{0} \in \mathbb{N}_{0}$ such that

$$
\gamma_{j+1} \leqslant d_{1} \gamma_{j} \text { for any } j \geqslant J_{0}
$$

Remark 2.2.2. It is easy to see that each sequence $\gamma=\left(\gamma_{j}\right)_{j \in \mathbb{N}_{0}}$, with the property that there is a constant $\lambda_{0}>1$ such that

$$
\lambda_{0} \gamma_{j} \leqslant \gamma_{j+1} \text { for all } j \in \mathbb{N}
$$

is strongly increasing in the sense of the above definition. However, not every strongly increasing sequence satisfies property (2.1).

Example 2.2.3. The sequence $\gamma=\left(\gamma_{j}\right)_{j \in \mathbb{N}_{0}}$ with $\gamma_{j}=2^{j \delta}(1+j)^{b}$, where $\delta>0$, $b \in \mathbb{R}$, is strongly increasing and of bounded growth, whereas $\gamma=\left(\gamma_{j}\right)_{j \in \mathbb{N}_{0}}$ with $\gamma_{j}=j$ ! is strongly increasing but not of bounded growth. Finally, the sequence $\gamma=\left(\gamma_{j}\right)_{j \in \mathbb{N}_{0}}$, with $\gamma_{j}=j$, is not strongly increasing, but of bounded growth.

As it was already mentioned in the Introduction, in the function spaces we will consider in this work we will have two parameters determining the generalised smoothness.

First we will deal with a sequence $N=\left(N_{j}\right)_{j \in \mathbb{N}_{0}}$ which will be strongly increasing in the next section and additionally of bounded growth in the main theorems of this work (local means and atomic decompositions).

Secondly, we will consider a sequence $\sigma=\left(\sigma_{j}\right)_{j \in \mathbb{N}_{0}}$ which can be considered as a smoothness weight on the different functions $f_{j}$, which are the result of the decomposition on the Fourier side. This sequence will fulfill

$$
d_{0} \sigma_{j} \leqslant \sigma_{j+1} \leqslant d_{1} \sigma_{j} \text { for all } j \in \mathbb{N},
$$

with two positive constants $d_{0}$ and $d_{1}$. In other words, both $\left(\sigma_{j}\right)_{j \in \mathbb{N}_{0}}$ and $\left(\sigma_{j}^{-1}\right)_{j \in \mathbb{N}_{0}}$ are of bounded growth.

Sequences $\sigma$ satisfying (2.2) will be called admissible sequences.

To illustrate the flexibility of the last condition we give some examples: 
Example 2.2.4. The sequence $\sigma=\left(\sigma_{j}\right)_{j \in \mathbb{N}_{0}}$,

$$
\sigma_{j}=2^{j s}(1+j)^{b}(1+\log (1+j))^{c},
$$

with arbitrary fixed real numbers $s, b$ and $c$, is the standard example of an admissible sequence and it can be considered in some sense as a model sequence. However, as it is shown in the next examples, the general definition also includes other sequences, which cannot be reduced to the one above or to a similar one.

For any $s \in \mathbb{R}$ we will denote

$$
\sigma^{s}=\left(2^{j s}\right)_{j \in \mathbb{N}_{0}} .
$$

Of course, $\sigma^{s}$ is a special case of (2.3) with $b=0$ and $c=0$. In particular $\sigma^{0}$ will denote the sequence with all terms equal with 1 .

Example 2.2.5. Let $s \in \mathbb{R}$ be fixed and

$$
\sigma_{j}=2^{j s} \Psi\left(2^{-j}\right), \quad j \in \mathbb{N}_{0},
$$

where $\Psi$ is a positive monotone function on $(0,1]$ and there are positive constants $b_{0}$ and $b_{1}$ such that, for all $j \in \mathbb{N}_{0}$,

$$
b_{0} \Psi\left(2^{-j}\right) \leqslant \Psi\left(2^{-2 j}\right) \leqslant b_{1} \Psi\left(2^{-j}\right) .
$$

Then it is easy to see that $\sigma$ is an admissible sequence.

This example goes back to [EdTr99] and gives a qualitative description of the model case in Example 2.2.4 with fixed main order $2^{j s}$.

Example 2.2.6. Let $\left(j_{l}\right)_{l \in \mathbb{N}_{0}}$ be a strongly increasing sequence of natural numbers, defined recursively by

$$
j_{0}=0, \quad j_{1}=1, \quad j_{2 l}=2 j_{2 l-1}-j_{2 l-2}, \quad j_{2 l+1}=2^{j_{2 l}}, \quad l \in \mathbb{N} .
$$

The sequence $\left(\sigma_{j}\right)_{j \in \mathbb{N}_{0}}$ is defined by

$$
\sigma_{j}=\left\{\begin{array}{rll}
2^{j_{2 l}} & \text { if } & j_{2 l} \leqslant j<j_{2 l+1} \\
2^{j_{2 l}} 4^{\left(j-j_{2 l+1}\right)} & \text { if } \quad j_{2 l+1} \leqslant j<j_{2 l+2} .
\end{array}\right.
$$

Then $\sigma$ is an admissible sequence. Moreover, the sequence $\left(\sigma_{j}\right)_{j \in \mathbb{N}_{0}}$ oscillates between $(j)_{j \in \mathbb{N}_{0}}$ and $\left(2^{j}\right)_{j \in \mathbb{N}_{0}}$, i.e.

$$
j \leqslant \sigma_{j} \leqslant 2^{j}
$$

and there exist infinitely many $j^{\prime}$ and $j^{\prime \prime}$ such that $\sigma_{j^{\prime}}=j^{\prime}$ and $\sigma_{j^{\prime \prime}}=2^{j^{\prime \prime}}$, respectively.

Example 2.2.7. Let $s \in \mathbb{R}$ be fixed and $\left(\sigma_{j}\right)_{j \in \mathbb{N}_{0}}$ be the above sequence. If

$$
\tau_{j}=2^{j s} \sigma_{j},
$$


then $\tau$ is admissible. $\left(\tau_{j}\right)_{j \in \mathbb{N}_{0}}$ oscillates between $\left(j 2^{j s}\right)_{j \in \mathbb{N}_{0}}$ and $\left(2^{j(s+1)}\right)_{j \in \mathbb{N}_{0}}$, i.e.

$$
j 2^{j s} \leqslant \tau_{j} \leqslant 2^{j(s+1)}
$$

and, again, infinitely many $\tau_{j}$ equal the left-hand side or the right-hand side of the above double inequality.

Remark 2.2.8. We would like to point out that the last two examples, which are due to G.A. Kalyabin, show that an admissible sequence does not have necessarily a fixed main order. Consequently the class of admissible sequences is larger than the class described in Example 2.2.5.

\subsection{Decompositions}

For a fixed strongly increasing sequence $N=\left(N_{j}\right)_{j \in \mathbb{N}_{0}}$ and a fixed $J \in \mathbb{N}$ we define the associate covering $\Omega^{N, J}=\left(\Omega_{j}^{N, J}\right)_{j \in \mathbb{N}_{0}}$ of $\mathbb{R}^{n}$ by

$$
\Omega_{j}^{N, J}=\left\{\xi \in \mathbb{R}^{n}:|\xi| \leqslant N_{j+J \kappa_{0}}\right\} \quad \text { if } \quad j=0,1, \ldots, J \kappa_{0}-1
$$

and

$$
\Omega_{j}^{N, J}=\left\{\xi \in \mathbb{R}^{n}: N_{j-J \kappa_{0}} \leqslant|\xi| \leqslant N_{j+J \kappa_{0}}\right\} \quad \text { if } \quad j=J \kappa_{0}, J \kappa_{0}+1, \ldots
$$

From the above definition it is obvious that each $\Omega_{j}^{N, J}$ has a non-empty intersection with at most $2(J+L+1) \kappa_{0}$ different sets $\Omega_{k}^{N, L}$ from a covering associated to the same sequence $N=\left(N_{j}\right)_{j \in \mathbb{N}_{0}}$.

For a fixed strongly increasing sequence $N=\left(N_{j}\right)_{j \in \mathbb{N}_{0}}$, a fixed $J \in \mathbb{N}$, and for the associated covering $\Omega^{N, J}=\left(\Omega_{j}^{N, J}\right)_{j \in \mathbb{N}_{0}}$ of $\mathbb{R}^{n}$, let $\Phi^{N, J}$ be the collection of all function systems $\varphi^{N, J}=\left(\varphi_{j}^{N, J}\right)_{j \in \mathbb{N}_{0}}$ such that:

(i) $\varphi_{j}^{N, J} \in C_{0}^{\infty}\left(\mathbb{R}^{n}\right)$ and $\varphi_{j}^{N, J}(\xi) \geqslant 0$ if $\xi \in \mathbb{R}^{n}$ for any $j \in \mathbb{N}_{0}$;

$$
\operatorname{supp} \varphi_{j}^{N, J} \subset \Omega_{j}^{N, J}
$$

(iii) for any $\gamma \in \mathbb{N}_{0}^{n}$ there exists a constant $c_{\gamma}>0$ such that for any $j \in \mathbb{N}_{0}$

$$
\left|D^{\gamma} \varphi_{j}^{N, J}(\xi)\right| \leqslant c_{\gamma}\langle\xi\rangle^{-\gamma} \text { for any } \xi \in \mathbb{R}^{n}
$$

(iv) there exists a constant $c_{\varphi}>0$ such that

$$
0<\sum_{j=0}^{\infty} \varphi_{j}^{N, J}(\xi)=c_{\varphi}<\infty \text { for any } \xi \in \mathbb{R}^{n} .
$$

By the relatively free choice of the sequence $\left(N_{j}\right)_{j \in \mathbb{N}_{0}}$ the construction of function systems $\left(\varphi_{j}\right)_{j \in \mathbb{N}_{0}}$ satisfying properties (2.7)-(2.10) is a little more complicated as in the classical case. We give a complete description in the following examples. 
Example 2.3.1. Let $\rho \in C_{0}^{\infty}(\mathbb{R})$ with $\rho(t)=1$ if $|t| \leqslant 1$, supp $\rho \subset\{t \in \mathbb{R}:|t| \leqslant 2\}$, and decreasing for $t \geqslant 0$.

A. Let

$$
\varphi_{j}^{N, J}(\xi)=\rho\left(N_{j}^{-1}|\xi|\right) \quad j=0,1, \ldots, J \kappa_{0}-1
$$

or

$$
\varphi_{J \kappa_{0}-1}^{N, J}(\xi)=\sum_{k=0}^{J \kappa_{0}-1} \rho\left(N_{k}^{-1}|\xi|\right), \quad \varphi_{j}^{N, J} \equiv 0 \text { if } j=0,1, \ldots, J \kappa_{0}-2
$$

and

$$
\varphi_{j}^{N, J}(\xi)=\rho\left(N_{j}^{-1}|\xi|\right)-\rho\left(N_{j-J \kappa_{0}}^{-1}|\xi|\right) \quad \text { for any } j \geqslant J \kappa_{0} .
$$

Then it is easy to see that the system $\varphi^{N, J}=\left(\varphi_{j}^{N, J}\right)_{j \in \mathbb{N}_{0}}$ satisfies (2.7)-(2.10) with $c_{\varphi}=\kappa_{0} J$.

B. Let also

$$
\psi_{k}^{N}(\xi)=\sum_{r=-(2 J+1) \kappa_{0}}^{(2 J+1) \kappa_{0}} \varphi_{k+r}^{N, J}(\xi) \quad \text { with } \quad \varphi_{-(2 J+1) \kappa_{0}}=\cdots=\varphi_{-1}=0 .
$$

Then $\left(\psi_{k}^{N}\right)_{k \in \mathbb{N}_{0}}$ is a function system which satisfies properties (i)-(iii) from above with respect to the covering $\Omega^{N, 3 J+2}$. This system has the useful property

$$
\psi_{k}^{N}(\xi)=c_{\varphi} \quad \text { on } \quad \operatorname{supp} \varphi_{k}^{N, J} .
$$

Moreover, if we define

$$
\widetilde{\psi}_{0}^{N}(\xi)=\psi_{0}^{N}(\xi)+\sum_{r=0}^{(2 J+1) \kappa_{0}-1}\left((2 J+1) \kappa_{0}-r\right) \varphi_{r}^{N, J}(\xi)
$$

then we have

$$
\widetilde{\psi}_{0}^{N}(\xi)+\sum_{k=1}^{\infty} \psi_{k}^{N}(\xi)=c_{\psi}=\left[(4 J+2) \kappa_{0}+1\right] c_{\varphi} .
$$

Remark 2.3.2. It is easy to see that if $\left(\varphi_{j}^{N, J}\right)_{j \in \mathbb{N}_{0}}$ fulfills (2.7)-(2.9) then for any multi-index $\alpha$ there is a constant $c_{\alpha}>0$ such that

$$
\sum_{j=0}^{\infty}\left|D^{\alpha} \varphi_{j}^{N, J}(\xi)\right| \leqslant\left[(2 J+1) \kappa_{0}\right] c_{\alpha}\langle\xi\rangle^{-|\alpha|}, \quad \text { for any } \xi \in \mathbb{R}^{n} .
$$

In particular, the last inequality implies

$$
\sup \left(R^{2|\alpha|-n} \int_{\frac{R}{2} \leqslant|\xi| \leqslant 2 R} \sum_{j=0}^{\infty}\left|D^{\alpha} \varphi_{j}^{N, J}(\xi)\right|^{2} d \xi\right)^{1 / 2}<\infty,
$$

where the supremum is taken over all $R>0$ and all multi-indices $\alpha$ with $0 \leqslant|\alpha| \leqslant$ $1+\left[\frac{n}{2}\right]$.

The same is true for the system $\left(\psi_{k}^{N, 3 J+2}\right)_{k \in \mathbb{N}_{0}}$ in the previous example. 


\section{Function spaces of generalised smoothness}

\subsection{The case $1<p<\infty$}

3.1.1. Definition and basic facts. For the definition of function spaces of generalised smoothness of Besov and Triebel-Lizorkin type for $1<p<\infty$, the main tool is the following classical Fourier-multiplier theorem of the MichlinHörmander type.

For a system $\left(m_{k, j}\right)_{k, j \in \mathbb{N}_{0}} \subset L_{\infty}\left(\mathbb{R}^{n}\right)$ let

$$
M=\sup \left(R^{2|\alpha|-n} \int_{\frac{R}{2} \leqslant|\xi| \leqslant 2 R} \sum_{k, j=0}^{\infty}\left|D^{\alpha} m_{k, j}(\xi)\right|^{2} d \xi\right)^{1 / 2},
$$

where the supremum is taken over all $R>0$ and all multi-indices $\alpha$ with $0 \leqslant|\alpha| \leqslant$ $1+\left[\frac{n}{2}\right]$.

Proposition 3.1.1. Let $1<p<\infty$ and $1<q<\infty$. Let $f=\left(f_{j}\right)_{j \in \mathbb{N}_{0}}$ be a system of measurable functions in $\mathbb{R}^{n}$.

(i) (General case) There exists a positive constant c such that

$$
\left\|\left(\sum_{j=0}^{\infty} m_{k, j}(D) f_{j}\right)_{k \in \mathbb{N}_{0}}\left|L_{p}\left(l_{2}\right)\|\leqslant c M \cdot\|\left(f_{j}\right)_{j \in \mathbb{N}_{0}}\right| L_{p}\left(l_{2}\right)\right\|,
$$

for all systems $\left(m_{k, j}\right)_{k, j \in \mathbb{N}_{0}} \subset L_{\infty}\left(\mathbb{R}^{n}\right)$.

(ii) (Diagonal case) There exist a positive constant c such that

$$
\left\|\left(m_{j, j}(D) f_{j}\right)_{j \in \mathbb{N}_{0}}\left|L_{p}\left(l_{q}\right)\|\leqslant c M \cdot\|\left(f_{j}\right)_{j \in \mathbb{N}_{0}}\right| L_{p}\left(l_{q}\right)\right\|,
$$

for all systems $\left(m_{k, j}\right)_{k, j \in \mathbb{N}_{0}} \subset L_{\infty}\left(\mathbb{R}^{n}\right)$ with $m_{k, j} \equiv 0$ if $k \neq j$.

A proof of the above result can be found in [Tr78, Theorem 2.2.4]. The first part is contained also in [Tr83, Equation 2.5.6/(1)].

In analogy to the classical case we introduce now function spaces of generalised smoothness of the Besov and Triebel-Lizorkin type.

Definition 3.1.2. Let $N=\left(N_{j}\right)_{j \in \mathbb{N}_{0}}$ be a strongly increasing sequence, not necessarily of bounded growth, let $J \in \mathbb{N}$, and let $\left(\varphi_{j}^{N, J}\right)_{j \in \mathbb{N}_{0}} \in \Phi^{N, J}$. Let $\left(\sigma_{j}\right)_{j \in \mathbb{N}_{0}}$ be an admissible sequence.

(i) Let $1<p<\infty, 1 \leqslant q \leqslant \infty$. Then the Besov space of generalised smoothness is

$$
B_{p, q}^{\sigma, N}=\left\{f \in 8^{\prime}:\left\|f\left|B_{p, q}^{\sigma, N}\|=\|\left(\sigma_{j} \varphi_{j}^{N, J}(D) f\right)_{j \in \mathbb{N}_{0}}\right| l_{q}\left(L_{p}\right)\right\|<\infty\right\} .
$$


(ii) Let $1<p<\infty, 1<q<\infty$. Then the Triebel-Lizorkin space of generalised smoothness is

$$
F_{p, q}^{\sigma, N}=\left\{f \in \mathcal{S}^{\prime}:\left\|f\left|F_{p, q}^{\sigma, N}\|=\|\left(\sigma_{j} \varphi_{j}^{N, J}(D) f(\cdot)\right)_{j \in \mathbb{N}_{0}}\right| L_{p}\left(l_{q}\right)\right\|<\infty\right\} .
$$

Note that if $N_{j}=2^{j}$ and $\sigma=\sigma^{s}=\left(2^{j s}\right)_{j \in \mathbb{N}_{0}}$ (recall notation (2.4) with $s$ real), then the above spaces coincide with the usual function spaces $B_{p, q}^{s}$ and $F_{p, q}^{s}$ on $\mathbb{R}^{n}$, respectively, systematically treated in the books of H. Triebel, see [Tr78], [Tr83], [Tr92] and [Tr01] and the references therein.

For sequences $\left(\sigma_{j}\right)_{j \in \mathbb{N}_{0}}$ with $\left(\sigma_{j}^{-1}\right)_{j \in \mathbb{N}_{0}} \in l_{q^{\prime}}$, where $q^{\prime}=q /(q-1)$, G.A. Kalyabin gave in [Ka80] a similar characterisation for such spaces, defined initially by approximation; for more details see Section 3.3.

Remark 3.1.3. Both $B_{p, q}^{\sigma, N}$ and $F_{p, q}^{\sigma, N}$ are Banach spaces which are independent of the choice of the system $\left(\varphi_{j}^{N, J}\right)_{j \in \mathbb{N}_{0}}$, in the sense of equivalent norms (and this is the reason why we may omit in our notation the subscript $\left.\left(\varphi_{j}^{N, J}\right)_{j \in \mathbb{N}_{0}}\right)$.

This can be shown in the standard way; compare for example [Tr78, Theorem 2.3.2] or [Tr83, Proposition 2.3.2/1].

Let us consider two different function systems $\left(\varphi_{j}^{N, J}\right)_{j \in \mathbb{N}_{0}}$ and $\left(\widetilde{\varphi}_{j}^{N, L}\right)_{j \in \mathbb{N}_{0}}$ related to the same strongly increasing sequence $N$.

Clearly for a fixed $j_{0} \in \mathbb{N}_{0}$ the intersection $\operatorname{supp} \varphi_{j_{0}}^{N, J} \cap \operatorname{supp} \widetilde{\varphi}_{k}^{N, L}$ is non-empty at most for $k$ in between $j_{0}-(L+J+1) \kappa_{0}$ and $j_{0}+(L+J+1) \kappa_{0}$.

The desired equivalence result is a simple consequence of the second part of Proposition 3.1.1, (diagonal case $-m_{k, j}=0$ if $k \neq j$ ) which is based on (2.11). In the case of Besov spaces, we use a scalar version - the classical Michlin-Hörmander Fourier-multiplier theorem for $L_{p}$ spaces.

As in the classical case, compare [Tr78, Theorem 2.3.2] or [Tr83, Proposition 2.3.3], the embeddings $\delta \hookrightarrow B_{p, q}^{\sigma, N} \hookrightarrow \delta^{\prime}$ and $\delta \hookrightarrow F_{p, q}^{\sigma, N} \hookrightarrow \delta^{\prime}$ hold true for all admissible values of the parameters and sequences. If $q<\infty$ then $\&$ is dense in $B_{p, q}^{\sigma, N}$ and in $F_{p, q}^{\sigma, N}$.

Moreover, it is clear that $B_{p, p}^{\sigma, N}=F_{p, p}^{\sigma, N}$.

If the sequences $\left(\sigma_{j}\right)_{j \in \mathbb{N}_{0}}$ have additionally the property $\left(\sigma_{j}^{-1}\right)_{j \in \mathbb{N}_{0}} \in l_{q^{\prime}}$, then all elements of $B_{p, q}^{\sigma, N}$ and of $F_{p, q}^{\sigma, N}$ are at least functions in $L_{p}$.

In this case many different results are already known from the works of G.A. Kalyabin and M.L. Goldman. We mention here only one final and remarkable embedding result, proved first in [Ka81].

Proposition 3.1.4. Let $1<p<\infty$ and $1<q<\infty$. Let also $N=\left(N_{j}\right)_{j \in \mathbb{N}_{0}}$ be a strongly increasing sequence and let $\left(\sigma_{j}\right)_{j \in \mathbb{N}_{0}}$ be an admissible sequence with $\left(\sigma_{j}^{-1}\right)_{j \in \mathbb{N}_{0}} \in l_{q^{\prime}}$. Then the following assertions are equivalent: 
(i) $F_{p, q}^{\sigma, N}$ is embedded into $C\left(\mathbb{R}^{n}\right), \quad\left(B_{p, q}^{\sigma, N}\right.$ is embedded into $\left.C\left(\mathbb{R}^{n}\right)\right)$

(ii) $F_{p, q}^{\sigma, N}$ is a multiplication algebra, $\left(B_{p, q}^{\sigma, N}\right.$ is a multiplication algebra);

(iii) $\left(\sigma_{j}^{-1} N_{j}^{n / p}\right)_{j \in \mathbb{N}_{0}} \in l_{p^{\prime}}, \quad\left(\left(\sigma_{j}^{-1} N_{j}^{n / p}\right)_{j \in \mathbb{N}_{0}} \in l_{q^{\prime}}\right)$;

respectively.

For embeddings into $L_{q}$-spaces, but also into Lorentz and Orlicz spaces we refer to [Go84a], [Go84b], [Go85], or to [Go92] for embeddings in a more complicated context.

Because the Fourier analytic approach allows us to consider also spaces of non-positive smoothness, we can obtain, and this is done in the rest of the section, results similar to those in the classical case.

\section{A Littlewood-Paley-type theorem}

Theorem 3.1.5. Let $1<p<\infty$ and $N=\left(N_{j}\right)_{j \in \mathbb{N}_{0}}$ be a strongly increasing sequence. Recall $\sigma^{0}$ denotes the sequence with all terms equal 1. Then

$$
F_{p, 2}^{\sigma^{0}, N}=L_{p}
$$

Proof. The proof is similar to that from [Tr83, Theorem 2.5.6] and it is based on the first part of Proposition 3.1.1 so that we will only sketch it.

If $f \in L_{p}$ we take, for any $k \in \mathbb{N}_{0}$, the function $m_{k, 0}=\varphi_{k}^{N, J}$ and $m_{k, j}=0$ if $j \geqslant 1$. We apply (3.1) with $f_{0}=f$ and $f_{j}=0$ if $j \geqslant 1$, and get $f \in F_{p, 2}^{\sigma^{0}, N}$ and $\left\|f\left|F_{p, 2}^{\sigma^{0}, N}\|\leqslant c\| f\right| L_{p}\right\|$.

To prove the reverse inequality, let $f \in F_{p, 2}^{\sigma^{0}, N}$ and let, for any $k \in \mathbb{N}_{0}$ as in Example 2.3.1,

$$
\psi_{k}^{N}(\xi)=\sum_{r=-(2 J+1) \kappa_{0}}^{(2 J+1) \kappa_{0}} \varphi_{k+r}^{N, J}(\xi) \quad \text { with } \quad \varphi_{-(2 J+1) \kappa_{0}}=\ldots=\varphi_{-1}=0 .
$$

Clearly $\psi_{k}^{N}(\xi)=c_{\varphi}$ if $\xi \in \operatorname{supp} \varphi_{k}^{N, J}$. Taking, for any $j \in \mathbb{N}_{0}$, the function $m_{0, j}=\psi_{j}^{N, J}$ and $m_{k, j}=0$ if $k \geqslant 1$, we apply (3.1) with $f_{j}=\varphi_{j}^{N, J}(D) f$ and get

$$
\begin{aligned}
\left(c_{\varphi}\right)^{-2}\left\|f \mid L_{p}\right\|= & \left\|\left(\delta_{k, 0} \sum_{j=0}^{\infty} \psi_{j}^{N}(D) \varphi_{j}^{N, J}(D) f\right)_{k \in \mathbb{N}_{0}} \mid L_{p}\left(l_{2}\right)\right\| \\
& \leqslant c\left\|\left(\varphi_{j}^{N, J}(D) f\right)_{j \in \mathbb{N}_{0}} \mid L_{p}\left(l_{2}\right)\right\|,
\end{aligned}
$$

and consequently $\left\|f\left|L_{p}\|\leqslant c\| f\right| F_{p, 2}^{\sigma^{0}, N}\right\|$, which proves the reverse inclusion.

Corollary 3.1.6. If $N=\left(N_{j}\right)_{j \in \mathbb{N}_{0}}$ is a strongly increasing sequence then $B_{2,2}^{\sigma^{0}, N}$ $=L_{2}$.

This can also be proved directly using the definition of the space $B_{2,2}^{\sigma^{0}, N}$. 


\section{Embeddings on the level of zero-smoothness}

On the level of zero-smoothness we have the following embeddings with respect to the usual Besov spaces $B_{p, 1}^{0}$ and $B_{p, \infty}^{0}$.

Theorem 3.1.7. Let $N=\left(N_{j}\right)_{j \in \mathbb{N}_{0}}$ be a strongly increasing sequence and let $1<p<\infty$.

(i) Then

$$
L_{p} \hookrightarrow B_{p, \infty}^{\sigma^{0}, N} \hookrightarrow B_{p, \infty}^{0} \quad \text { and } \quad B_{p, 1}^{0} \hookrightarrow B_{p, 1}^{\sigma^{0}, N} \hookrightarrow L_{p} .
$$

(ii) If, in addition, the sequence $N=\left(N_{j}\right)_{j \in \mathbb{N}_{0}}$ is of bounded growth then, for any $1 \leqslant q \leqslant \infty$,

$$
B_{p, q}^{\sigma^{0}, N}=B_{p, q}^{0} .
$$

Proof. Although the proof is in some sense straightforward, we will give a short outline. The main point is, that we compare here decompositions with respect to different sequences $\left(N_{j}\right)_{j \in \mathbb{N}_{0}}$ and $\left(2^{k}\right)_{k \in \mathbb{N}_{0}}$.

We can assume that the parameter $J$ in the definition of the coverings equals 1 in both cases. Let $\left(\varphi_{j}^{N}\right)_{j \in \mathbb{N}_{0}}$ be a function system belonging to the covering associated to $\left(N_{j}\right)_{j \in \mathbb{N}_{0}}$ and $\left(\psi_{k}\right)_{k \in \mathbb{N}_{0}}$ a system belonging to the covering associated to $\left(2^{k}\right)_{k \in \mathbb{N}_{0}}$, respectively. If $k_{0}$ is fixed, then

$$
\operatorname{supp} \varphi_{j}^{N} \cap \operatorname{supp} \psi_{k_{0}} \neq \varnothing
$$

at most for $4 \kappa_{0}+1$ indices $j$.

On the other hand, fixing $j_{0}$, we find out that

$$
\operatorname{supp} \varphi_{j_{0}}^{N} \cap \operatorname{supp} \psi_{k} \neq \varnothing
$$

is possible in general for all indices $k$ in between $\log \left(N_{j_{0}-\kappa_{0}} / 2\right)$ and $\log \left(2 N_{j_{0}+\kappa_{0}}\right)$. This means, the number of indices can increase and tends to infinity, if $\log \left(4 \frac{N_{j_{0}+\kappa_{0}}}{N_{j_{0}-\kappa_{0}}}\right)$ is not bounded.

Now let $f \in L_{p}$. Then by the scalar Fourier-multiplier theorem in $L_{p}$ we have

$$
\begin{aligned}
\left\|f \mid B_{p, \infty}^{\sigma^{0}, N}\right\| & =\sup _{j \in \mathbb{N}_{0}}\left\|\varphi_{j}^{N}(D) f \mid L_{p}\right\| \\
& \leqslant \sup _{j \in \mathbb{N}_{0}}\left\|\varphi_{j}^{N}(D)\left|\mathcal{L}\left(L_{p}\right)\|\| f\right| L_{p}\right\| \leqslant c\left\|f \mid L_{p}\right\|,
\end{aligned}
$$

where $\mathcal{L}\left(L_{p}\right)$ is the space of linear bounded operators from $L_{p}$ into itself.

If $f \in B_{p, q}^{\sigma^{0}, N}$, then

$$
\begin{aligned}
\left\|f \mid B_{p, \infty}^{0}\right\| & =\sup _{k \in \mathbb{N}_{0}}\left\|\psi_{k}(D) f\left|L_{p}\left\|=\left(c_{\varphi}\right)^{-1} \sup _{k \in \mathbb{N}_{0}}\right\| \psi_{k}(D) \sum_{j=0}^{\infty} \varphi_{j}^{N}(D) f\right| L_{p}\right\| \\
& =\left(c_{\varphi}\right)^{-1} \sup _{k \in \mathbb{N}_{0}}\left\|\psi_{k}(D) \sum_{j=j(k)}^{j(k)+4 \kappa_{0}} \varphi_{j}^{N}(D) f \mid L_{p}\right\| \\
& \leqslant c \sup _{k \in \mathbb{N}_{0}}\left\|\psi_{k}(D)\left|\mathcal{L}\left(L_{p}\right)\left\|\left(4 \kappa_{0}+1\right) \sup _{j \in \mathbb{N}_{0}}\right\| \varphi_{j}^{N}(D) f\right| L_{p}\right\| \\
& \leqslant c^{\prime}\left\|f \mid B_{p, \infty}^{\sigma^{0}, N}\right\| .
\end{aligned}
$$


We omit the proof of the second inclusion in (i).

To prove the second part, we recall that, if the sequence $\left(N_{j}\right)_{j \in \mathbb{N}_{0}}$ is of bounded growth, then we have

$$
\frac{N_{j_{0}+\kappa_{0}}}{N_{j_{0}-\kappa_{0}}} \leqslant\left(d_{1}^{(N)}\right)^{2 \kappa_{0}} .
$$

Consequently for these sequences there are finite universal upper bounds for the cardinality of indices $k$ such that $\operatorname{supp} \psi_{k}$ and $\operatorname{supp} \varphi_{j_{0}}^{N}$ can have a non-empty intersection. So we get

$$
\begin{aligned}
\left\|f \mid B_{p, q}^{\sigma^{0}, N}\right\|^{q} & =\sum_{j=0}^{\infty}\left\|\varphi_{j}^{N}(D) f \mid L_{p}\right\|^{q} \\
& \leqslant\left(c_{\psi}\right)^{-1} \sum_{j=0}^{\infty}\left\|\varphi_{j}^{N}(D) \sum_{k=k(j)}^{k(j)+K} \psi_{k}(D) f \mid L_{p}\right\|^{q} \\
& \leqslant c \sup _{j \in \mathbb{N}_{0}}\left\|\varphi_{j}^{N}(D)\left|\mathcal{L}\left(L_{p}\right)\left\|\sum_{j=0}^{\infty} \sum_{k=k(j)}^{k(j)+K}\right\| \psi_{k}(D) f\right| L_{p}\right\|^{q} \\
& \leqslant c^{\prime}\left\|f \mid B_{p, q}^{0}\right\|^{q},
\end{aligned}
$$

where the constants $c$ and $c^{\prime}$ depend, of course, on $K, \kappa_{0}$ and $q$. The main point is, that each $\psi_{k}(D) f$ can appear at most $4 \kappa_{0}+1$ times.

The reverse estimate can be proved in the same way, changing the roles of the function systems and of $K$ and $4 \kappa_{0}+1$, respectively.

Remark 3.1.8. The results stated in the above theorem are sharp.

To see this, we will show that if the sequence $N$ is $N_{j}=j$ ! (for any $j$ ) then we have, even in the case $p=2$,

$$
B_{2, \infty}^{\sigma^{0}, N} \hookrightarrow B_{2, \infty}^{0} \quad \text { and } \quad B_{2, \infty}^{\sigma^{0}, N} \neq B_{2, \infty}^{0} .
$$

To prove this, again let $\left(\psi_{k}\right)_{k \in \mathbb{N}_{0}}$ be a system belonging to the covering associated to $\left(2^{k}\right)_{k \in \mathbb{N}_{0}}$ - as in Example 2.3.1.A. defined by $\psi_{k}(\xi)=\rho\left(2^{-k}|\xi|\right)-$ $\rho\left(2^{-k+1}|\xi|\right)$, where $\rho \in C_{0}^{\infty}(\mathbb{R})$ with $\rho(t)=1$ if $|t|<1$ and $\rho(t)=0$ if $|t| \geqslant 2$.

Consequently, the special sequence leads to $\psi_{k}(\xi)=\psi_{1}\left(2^{-k+1} \xi\right)$ for $k \geqslant 1$.

Let $u$ be such that

$$
\widehat{u}(\xi)=\sum_{k=0}^{\infty} 2^{-k \frac{n}{2}} \psi_{k}(\xi) .
$$

We will show that

$$
u \in B_{2, \infty}^{0} \quad \text { and } \quad u \notin B_{2, \infty}^{\sigma^{0}, N} .
$$

One has, for any $k \in \mathbb{N}_{0}$,

$$
\begin{aligned}
\left\|\psi_{k}(D) u \mid L_{2}\right\| & =\left\|\psi_{k} \widehat{u}\left|L_{2}\|=\| \sum_{j=k-1}^{k+1} 2^{-j \frac{n}{2}} \psi_{k} \psi_{j}\right| L_{2}\right\| \\
& \leqslant c \cdot 2^{-k \frac{n}{2}}\left\|\psi_{k} \mid L_{2}\right\|
\end{aligned}
$$


(with appropriate changes if $k=0$ ). Since

$$
\left\|\left.\psi_{k}\left|L_{2} \|^{2}=\int_{\mathbb{R}^{n}}\right| \psi_{1}\left(2^{-k+1} \xi\right)\right|^{2} d \xi=2^{(k-1) n} \int_{\mathbb{R}^{n}}\left|\psi_{1}(\xi)\right|^{2} d \xi=c_{\rho} 2^{k n},\right.
$$

we get that there exists a constant $C>0$ with

$$
\sup _{k \in \mathbb{N}_{0}}\left\|\psi_{k}(D) u\left|L_{2}\|=\| u\right| B_{2, \infty}^{0}\right\| \leqslant C
$$

and this proves the first part in (3.4). Now we will show that $u \notin B_{2, \infty}^{\sigma^{0}, N}$.

Let $\left(\chi_{k}^{N}\right)_{k \in \mathbb{N}_{0}}$ be a function system belonging to the covering associated to $(k !)_{k \in \mathbb{N}_{0}}$ as in Example 2.3.1.B. That is, we have additionally

$$
\chi_{k}^{N}(\xi)=1 \quad \text { on } \quad A_{k}=\left\{\xi \in \mathbb{R}^{n}:(k-1) !<|\xi|<(k+1) !\right\}
$$

and

$$
\operatorname{supp} \chi_{k}^{N} \subset\{\xi:(k-5) !<|\xi|<(k+5) !\} .
$$

Clearly this system gives an equivalent norm in $B_{2, \infty}^{\sigma^{0}, N}$, too. We have

$$
\begin{aligned}
\left\|\chi_{k}^{N}(D) u \mid L_{2}\right\|^{2} & =\left\|\left.\chi_{k}^{N} \widehat{u}\left|L_{2} \|^{2}=\int_{\mathbb{R}^{n}}\right| \chi_{k}^{N}(\xi) \cdot \sum_{j=0}^{\infty} 2^{-j \frac{n}{2}} \psi_{j}(\xi)\right|^{2} d \xi\right. \\
& \geqslant \int_{A_{k}}\left|\sum_{j=0}^{\infty} 2^{-j \frac{n}{2}} \psi_{j}(\xi)\right|^{2} d \xi \geqslant \sum_{l=0}^{\infty} 2^{-2 \ln } \int_{A_{k}}\left|\psi_{2 l}(\xi)\right|^{2} d \xi
\end{aligned}
$$

we consider only even indices since $\operatorname{supp} \psi_{2 l} \cap \operatorname{supp} \psi_{2(l+1)}=\emptyset$. Now we determine the number of indices $l$ such that supp $\psi_{2 l} \subset A_{k}=\left\{\xi \in \mathbb{R}^{n}:(k-1) !<\right.$ $|\xi|<(k+1)$ ! \}. Let $l_{0}$ be fixed in such a way that

$$
2^{2\left(l_{0}-1\right)-1}<(k-1) !<2^{2 l_{0}-1} ;
$$

in particular this implies supp $\psi_{2 l_{0}} \subset\left\{\xi \in \mathbb{R}^{n}:(k-1) !<|\xi|<(k+1)\right.$ ! $\}$ if $k \geqslant 4$ and $L$ is fixed such that

$$
2^{2\left(l_{0}+L\right)+1}<(k+1) !<2^{2\left(l_{0}+L+1\right)+1} .
$$

Then $L+1$ is the cardinality of those $l$ such that $\operatorname{supp} \psi_{2 l} \subset\left\{\xi \in \mathbb{R}^{n}:(k-1) !<\right.$ $|\xi|<(k+1)$ ! $\}$ is guaranteed. Using (3.6) and (3.7) it is easy to see that we get

$$
\log k+\log (k+1)<2 L+6 \text {. }
$$

For any $l$ in between $l_{0}$ and $l_{0}+L$ we have

$$
\int_{A_{k}}\left|\psi_{2 l}(\xi)\right|^{2} d \xi=\left\|\psi_{2 l} \mid L_{2}\right\|^{2}=c_{\rho} 2^{2 l n}
$$

and this leads to

$$
\left\|\chi_{k}^{N}(D) u \mid L_{2}\right\|^{2} \geqslant \sum_{l=l_{0}}^{l_{0}+L} c_{\rho} \geqslant c_{\rho}(L+1) \geqslant c_{\rho}(\log k-2),
$$


and consequently to

$$
\left\|u\left|B_{2, \infty}^{\sigma^{0}, N}\left\|=\sup _{k \in \mathbb{N}_{0}}\right\| \chi_{k}^{N}(D) u\right| L_{2}\right\|=\infty,
$$

which proves our statement.

\section{Existence of a lift operator}

In the next theorem we show the existence of a lift operator between the spaces of $B_{p, q}^{\sigma, N}$ and $F_{p, q}^{\sigma, N}$ type.

Theorem 3.1.9. Let $\left(\sigma_{j}\right)_{j \in \mathbb{N}_{0}}$ and $\left(\beta_{j}\right)_{j \in \mathbb{N}_{0}}$ be two admissible sequences and let $\left(\varphi_{j}^{N, J}\right)_{j \in \mathbb{N}_{0}}$ be a function system associated to the strongly increasing sequence $\left(N_{j}\right)_{j \in \mathbb{N}_{0}}$.

Then the operator $\mu(D)$, defined by the symbol

$$
\mu(\xi)=\sum_{j=0}^{\infty} \sigma_{j} \beta_{j}^{-1} \varphi_{j}^{N, J}(\xi),
$$

defines, for all parameters $1<p<\infty$ and $1 \leqslant q \leqslant \infty$, an isomorphism between $B_{p, q}^{\sigma, N}$ and $B_{p, q}^{\beta, N}$, respectively, between $F_{p, q}^{\sigma, N}$ and $F_{p, q}^{\beta, N}$.

Proof. The symbol is well defined and smooth, because, for fixed $\xi$, at most $(4 J+2) \kappa_{0}$ terms in the infinite sum are not zero.

It is easy to see that, due to the construction and the properties of the sequences $\sigma$ and $\beta$ on $\operatorname{supp} \varphi_{k}^{N, J}$, one has

$$
\left|\chi_{\text {supp } \varphi_{k}^{N, J}}(\xi) D^{\alpha} \mu(\xi)\right| \leqslant \max _{|r| \leqslant(2 J+1) \kappa_{0}}\left(\sigma_{k+r} \beta_{k+r}^{-1}\right)\langle\xi\rangle^{-|\alpha|} \leqslant c \sigma_{k} \beta_{k}^{-1}\langle\xi\rangle^{-|\alpha|}
$$

and

$$
c^{\prime} \sigma_{k} \beta_{k}^{-1} \leqslant \min _{|r| \leqslant(2 J+1) \kappa_{0}}\left(\sigma_{k+r} \beta_{k+r}^{-1}\right) \leqslant\left|\chi_{\text {supp } \varphi_{k}^{N, J}}(\xi) \mu(\xi)\right| .
$$

The rest of the proof is a standard application of Definition 3.1.2 of the spaces $B_{p, q}^{\sigma, N}$ and $F_{p, q}^{\sigma, N}$ using the inequalities (3.8) and (3.9).

\section{Duality}

In the following theorem we determine the dual spaces of $B_{p, q}^{\sigma, N}$ and $F_{p, q}^{\sigma, N}$. The previous results - see the end of Remark 3.1.3 - give the possibility of interpreting the dual spaces $\left(B_{p, q}^{\sigma, N}\right)^{\prime}$ and $\left(F_{p, q}^{\sigma, N}\right)^{\prime}$ as subspaces of $\delta^{\prime}$. Furthermore, because $\delta$ is dense in these spaces if $q<\infty, f$ belongs to $\left(F_{p, q}^{\sigma, N}\right)^{\prime} \hookrightarrow \delta^{\prime}$ (similar for $\left.\left(B_{p, q}^{\sigma, N}\right)^{\prime} \hookrightarrow \AA^{\prime}\right)$, if, and only if, there is a number $c$ such that, for all $\psi \in \&$,

$$
|<f, \psi>| \leqslant c\left\|\psi \mid F_{p, q}^{\sigma, N}\right\| .
$$

For an admissible sequence $\sigma=\left(\sigma_{j}\right)_{j \in \mathbb{N}_{0}}$ we denote $1 / \sigma=\left(1 / \sigma_{j}\right)_{j \in \mathbb{N}_{0}}$. Clearly $1 / \sigma$ is also admissible. 
Theorem 3.1.10. Let $N=\left(N_{j}\right)_{j \in \mathbb{N}_{0}}$ be a strongly increasing sequence and $\left(\sigma_{j}\right)_{j \in \mathbb{N}_{0}}$ be an admissible sequence. Furthermore, let $1<p<\infty, 1 \leqslant q<\infty$ and let $p^{\prime}$ and $q^{\prime}$ denote their conjugates.

Then

$$
\left(B_{p, q}^{\sigma, N}\right)^{\prime}=B_{p^{\prime}, q^{\prime}}^{1 / \sigma, N} \text { and }\left(F_{p, q}^{\sigma, N}\right)^{\prime}=F_{p^{\prime}, q^{\prime}}^{1 / \sigma, N}
$$

Proof. The proof follows essentially that of [Tr83, Theorem 2.11.2]. First we prove

$$
F_{p^{\prime}, q^{\prime}}^{1 / \sigma, N} \hookrightarrow\left(F_{p, q}^{\sigma, N}\right)^{\prime}
$$

Let $f \in F_{p^{\prime}, q^{\prime}}^{1 / \sigma, N}$ and let $\left(\varphi_{j}^{N, J}\right)_{j \in \mathbb{N}_{0}}$ and $\left(\psi_{k}^{N}\right)_{k \in \mathbb{N}_{0}}$ be the systems from Example 2.3.1, that is

$$
\psi_{k}^{N}(\xi)=\sum_{r=-(2 J+1) \kappa_{0}}^{(2 J+1) \kappa_{0}} \varphi_{k+r}^{N, J}(\xi) \quad \text { and } \quad \psi_{k}^{N}(\xi)=c_{\varphi} \text { on } \operatorname{supp} \varphi_{k}^{N, J}
$$

Then

$$
\left(c_{\varphi}\right)^{2} f=\sum_{k=0}^{\infty} \varphi_{k}^{N, J}(D) \psi_{k}^{N}(D) f \quad \text { in } \quad \varsigma^{\prime}
$$

and

$$
\left\|\left(\sigma_{k}^{-1} \psi_{k}^{N}(D) f\right)_{k \in \mathbb{N}_{0}}\left|L_{p^{\prime}}\left(l_{q^{\prime}}\right)\|\leqslant c\| f\right| F_{p^{\prime}, q^{\prime}}^{1 / \sigma, N}\right\| .
$$

If $\psi \in \S$, we have

$$
\begin{aligned}
& \left(c_{\varphi}\right)^{2}|<f, \psi>| \\
= & \left|\sum_{k=0}^{\infty} \sum_{r=-(2 J+1) \kappa_{0}}^{(2 J+1) \kappa_{0}}<\psi_{k}^{N}(D) f, c_{\varphi}^{-1} \mathcal{F} \varphi_{k}^{N, J}(\xi) \varphi_{k+r}^{N, J}(\xi) \mathcal{F}^{-1} \psi>\right| \\
\leqslant & c \sum_{r=-(2 J+1) \kappa_{0}}^{(2 J+1) \kappa_{0}}\left\|\left(\sigma_{k}^{-1} \psi_{k}^{N}(D) f\right)_{k \in \mathbb{N}_{0}} \mid L_{p^{\prime}}\left(l_{q^{\prime}}\right)\right\| \times \\
\leqslant & c^{\prime}\left\|f\left|F_{p^{\prime}, q^{\prime}}^{1 / \sigma, N}\|\| \psi\right| \sigma_{k} \mathcal{F} \varphi_{k}^{N, J}(\xi) \varphi_{k+r}^{N, J}(\xi) F_{p, q}^{\sigma-N} \psi .\right.
\end{aligned}
$$

In view of (3.10) this proves (3.11).

We will prove the reverse embedding. Because, for $f \in F_{p, q}^{\sigma, N}$, the mapping

$$
f \mapsto\left(\sigma_{j} \varphi_{j}^{N, J}(D) f\right)_{j \in \mathbb{N}_{0}}
$$

is a one-to-one mapping onto a subspace of $L_{p}\left(l_{q}\right)$, every functional $g \in\left(F_{p, q}^{\sigma, N}\right)^{\prime}$ can be interpreted as a functional on that subspace. By the Hahn-Banach theorem, $g$ can be extended to a continuous linear functional $\bar{g}$ on the whole space $L_{p}\left(l_{q}\right)$, where the norm is preserved. But the representation of these linear functionals is 
well known. Proposition 2.11.1 in [Tr83] gives an exact characterisation and yields to

$$
<\bar{g}, f>=\sum_{j=0}^{\infty} \int_{\mathbb{R}^{n}} \sigma_{j}^{-1} g_{j}(x) \sigma_{j} f_{j}(x) d x,
$$

for every $f=\left(\sigma_{j} f_{j}\right)_{j \in \mathbb{N}_{0}} \in L_{p}\left(l_{q}\right)$, where $\left(\sigma_{j}^{-1} g_{j}\right)_{j \in \mathbb{N}_{0}} \in L_{p^{\prime}}\left(l_{q^{\prime}}\right)$ and

$$
\left\|g\left|\left(F_{p, q}^{\sigma, N}\right)^{\prime}\|=\| \bar{g}\right|\left(L_{p}\left(l_{q}\right)\right)^{\prime}\right\|=\left\|\left(\sigma_{j}^{-1} g_{j}\right)_{j \in \mathbb{N}_{0}} \mid L_{p^{\prime}}\left(l_{q^{\prime}}\right)\right\| .
$$

If again $\psi \in \S$, this gives, with $\psi \mapsto\left(\sigma_{j} \varphi_{j}^{N, J}(D) \psi\right)_{j \in \mathbb{N}_{0}}$,

$c_{\varphi}<\bar{g}, \psi>=\sum_{j=0}^{\infty} \int_{\mathbb{R}^{n}} g_{j}(x)\left(\varphi_{j}^{N, J}(D) \psi\right)(x) d x=<\sum_{j=0}^{\infty}\left(\mathcal{F} \varphi_{j}^{N, J}(\xi) \mathcal{F}^{-1} g_{j}\right), \psi>$.

So we obtain

$$
\begin{aligned}
\left\|g \mid F_{p^{\prime}, q^{\prime}}^{1 / \sigma, N}\right\| & =\left\|\left(\sigma_{k}^{-1} \varphi_{k}^{N, J}(D) \sum_{j=0}^{\infty}\left(\mathcal{F} \varphi_{j}^{N, J}(\xi) \mathcal{F}^{-1} g_{j}\right)\right)_{k \in \mathbb{N}_{0}} \mid L_{p^{\prime}}\left(l_{q^{\prime}}\right)\right\| \\
& \leqslant c \sum_{r=-(2 J+1) \kappa_{0}}^{(2 J+1) \kappa_{0}}\left\|\left(\sigma_{k}^{-1} \varphi_{k}^{N, J}(D)\left(\mathcal{F} \varphi_{k+r}^{N, J}(\xi) \mathcal{F}^{-1} g_{k+r}\right)\right)_{k \in \mathbb{N}_{0}} \mid L_{p^{\prime}}\left(l_{q^{\prime}}\right)\right\| \\
& \leqslant\left\|\left(\sigma_{k}^{-1} g_{k}\right)_{k \in \mathbb{N}_{0}} \mid L_{p^{\prime}}\left(l_{q^{\prime}}\right)\right\| \\
& \leqslant\left\|g \mid\left(F_{p, q}^{\sigma, N}\right)^{\prime}\right\| .
\end{aligned}
$$

The last estimate follows again by the second part of the Proposition 3.1.1 and the property of the sequence $\left(\sigma_{j}\right)_{j \in \mathbb{N}_{0}}$.

In the case of Besov spaces the proof can be given in a similar way.

3.1.2. Special classes: function spaces of generalised smoothness associated to an admissible symbol. In the previous subsection we have introduced and considered function spaces of generalised smoothness associated to a general strongly increasing sequence $N$ and to an admissible sequence $\sigma$.

In recent years there has been an increasing interest in investigating function spaces of general smoothness for which the strongly increasing sequence $N$ is associated (in a canonical way) to a fixed smooth function satisfying some reasonable conditions; we will call those smooth functions admissible symbols.

Definition 3.1.11. Let $\mathcal{A}$ be the class of all non-negative functions $a: \mathbb{R}^{n} \rightarrow \mathbb{R}$ of class $C^{\infty}$ with the following properties:

(i) $\lim _{|\xi| \rightarrow \infty} a(\xi)=\infty$;

(ii) a is almost increasing in $|\xi|$, i.e. there exists a constant $\delta_{0} \geqslant 1$, and an $R>0$ such that $a(\xi) \leqslant \delta_{0} a(\eta)$ if $R \leqslant|\xi| \leqslant|\eta|$; 
(iii) there exists an $m>0$ such that $\xi \mapsto a(\xi)|\xi|^{-m}$ is almost decreasing in $|\xi|$, i.e. there exists a constant $\delta_{m}, 0<\delta_{m} \leqslant 1$, and an $R>0$ such that

$$
a(\xi)|\xi|^{-m} \geqslant \delta_{m} a(\eta)|\eta|^{-m} \quad \text { if } \quad R \leqslant|\xi| \leqslant|\eta|
$$

(iv) for every multi-index $\alpha \in \mathbb{N}_{0}^{n}$ there exists some $c_{\alpha}>0$ such that

$$
\left|D^{\alpha} a(\xi)\right| \leqslant c_{\alpha} a(\xi)\langle\xi\rangle^{-|\alpha|} \quad \text { if } \quad|\xi| \geqslant R .
$$

The functions a from $\mathcal{A}$ are called admissible symbols.

Clearly the functions $\xi \mapsto|\xi|^{2}$ and $\xi \mapsto 1+|\xi|^{2}$ are admissible symbols.

It is easy to give further examples with the help of Bernstein functions; compare Corollary 3.1.14 below.

Recall that an arbitrarily often differentiable function $f:(0, \infty) \rightarrow \mathbb{R}$ with continuous extension to $[0, \infty)$ is called a Bernstein function if $f(t) \geqslant 0$ for all $t>0$ and $(-1)^{k} f^{(k)}(t) \leqslant 0$ for all $t>0$ and all $k \in \mathbb{N}$.

For any Bernstein function $f:(0, \infty) \rightarrow(0, \infty)$ one has, for any $j \in \mathbb{N}$,

$$
\left|f^{(j)}(t)\right| \leqslant \frac{j !}{t^{j}} f(t), \quad t>0 .
$$

In particular, for $j=1$ we have

$$
0 \leqslant f^{\prime}(t) \leqslant \frac{f(t)}{t} \text { for } t>0 .
$$

For more information on Bernstein functions the reader is referred to [Sc94], [Sc98a] or [Ja01]. Here we will restrict ourselves only to some examples.

Example 3.1.12. The function $t \mapsto c, c \geqslant 0$, is a Bernstein function as well as the function $t \mapsto b t, b \geqslant 0$. Moreover, $f(t)=1-e^{-r t}$, with fixed $r \geqslant 0$, is also obviously a Bernstein function.

For $\varrho \in[0,1]$ the function $f_{\varrho}(t)=t^{\varrho}$ is a Bernstein function.

The function $f(t)=\log (1+t)$ is also a Bernstein function.

For $m>0$ the function $f(t)=\sqrt{t+m^{2}}-m$ is a Bernstein function.

The functions $f(t)=\sqrt{t} \log (1+\sqrt{t}), f(t)=\sqrt{t}(1-\exp (-4 \sqrt{t})), f(t)=$ $\sqrt{t} \log (1+\operatorname{coth} \sqrt{t})$ and $f(t)=\frac{t}{t+\lambda}$, with $\lambda>0$, are further examples of Bernstein functions.

Lemma 3.1.13. If $f$ is a Bernstein function and $a: \mathbb{R}^{n} \rightarrow \mathbb{R}$ is a non-negative function satisfying (3.12) then $b(\xi)=f(a(\xi))$ satisfies (3.12).

Proof. To show (3.12) for the function $b$ let us recall that for the arbitrarily often differentiable functions $f:(0, \infty) \rightarrow(0, \infty)$ and $a: \mathbb{R}^{n} \rightarrow(0, \infty)$ and for any $\alpha \in \mathbb{N}_{0}^{n}$ one has

$$
D^{\alpha}(f \circ a)=\sum_{j=1}^{|\alpha|} f^{(j)}(a(\cdot)) \sum \frac{\alpha !}{\delta_{\beta} ! \delta_{\gamma} ! \cdot \ldots \cdot \delta_{\omega} !}\left(\frac{D^{\beta} a(\cdot)}{\beta !}\right)^{\delta_{\beta}} \cdot \ldots \cdot\left(\frac{D^{\omega} a(\cdot)}{\omega !}\right)^{\delta_{\omega}}
$$


where the second sum extends over all pairwise different multi-indices $0 \neq$ $\beta, \gamma, \ldots, \omega \in \mathbb{N}_{0}^{n}$ and all $\delta_{\beta}, \delta_{\gamma}, \ldots, \delta_{\omega} \in \mathbb{N}$ such that $\delta_{\beta} \beta+\delta_{\gamma} \gamma+\cdots+\delta_{\omega} \omega=\alpha$ and $\delta_{\beta}+\delta_{\gamma}+\cdots+\delta_{\omega}=j$.

Using (3.13) and the fact that $a$ satisfies (3.12), we get, for any $\alpha \in \mathbb{N}_{0}^{n}$,

$$
\begin{aligned}
\left|D^{\alpha} b(\xi)\right| & \leqslant \sum_{j=1}^{|\alpha|} \frac{j !}{a(\xi)^{j}} f(a(\xi)) \sum \frac{\alpha !}{\delta_{\beta} ! \delta_{\gamma} ! \cdot \ldots \cdot \delta_{\omega} !}\left|\frac{D^{\beta} a(\xi)}{\beta !}\right|^{\delta_{\beta}} \cdot \ldots \cdot\left|\frac{D^{\omega} a(\xi)}{\omega !}\right|^{\delta_{\omega}} \\
& \leqslant c_{\alpha} \sum_{j=1}^{|\alpha|} \frac{j !}{a(\xi)^{j}} f(a(\xi)) \prod_{\beta} a(\xi)^{\delta_{\beta}}\langle\xi\rangle^{-\delta_{\beta}|\beta|} \\
& \leqslant c_{\alpha}^{\prime} f(a(\xi))\left(1+|\xi|^{2}\right)^{-\frac{|\alpha|}{2}}
\end{aligned}
$$

and this completes the proof. For a similar calculation one can see also [JaSc96].

The next corollary is a simple consequence of the above lemma, of the monotonicity of Bernstein functions, and of property (3.14).

Corollary 3.1.14. For any Bernstein function $f$ with $\lim _{t \rightarrow \infty} f(t)=\infty$ the function $b(\xi)=f\left(1+|\xi|^{2}\right)$ is an admissible symbol.

If, in addition, $f \in C^{\infty}([0, \infty))$ then the function $b(\xi)=f\left(|\xi|^{2}\right)$ is also an admissible symbol.

As a simple consequence we obtain:

Example 3.1.15. The functions

$$
\begin{aligned}
& a(\xi)=\langle\xi\rangle^{2 \varrho}, \quad \varrho \in[0,1], \\
& a(\xi)=\log \left(1+|\xi|^{2}\right), \\
& a(\xi)=\sqrt{|\xi|^{2}+m^{2}}-m, \quad m>0, \\
& a(\xi)=\langle\xi\rangle \log (1+\langle\xi\rangle), \\
& a(\xi)=\langle\xi\rangle(1-\exp (-4\langle\xi\rangle)), \\
& a(\xi)=\langle\xi\rangle \log (1+\operatorname{coth}\langle\xi\rangle),
\end{aligned}
$$

are admissible symbols.

Note that if $f, g$ are two Bernstein functions then obviously $f \circ g$ is also a Bernstein function.

Consequently, using Corollary 3.1.14 we obtain many non-trivial examples of admissible symbols $a$ of the form $f\left(|\xi|^{2}\right)$ or $f\left(1+|\xi|^{2}\right)$, with $f$ a Bernstein function satisfying $\lim _{t \rightarrow \infty} f(t)=\infty$.

Remark 3.1.16. Properties (i) and (iv) from Definition 3.1.11 guarantee the hypoellipticity of the function $a$.

If $\delta_{0}=1$ then the function $a$ is radial symmetric and increasing. Moreover, if $\delta_{m}=1$ then $a$ is radial symmetric, too. 
We want to point out that the class $\mathcal{A}$ is larger than the class $S^{*}\left(m, m^{\prime}, 0\right)$ considered in [Le90]. For $S^{*}\left(m, m^{\prime} ; 0\right)$ it was additionally required that there exists an $m^{\prime}>0$, such that $a(\xi)|\xi|^{-m^{\prime}}$ is almost increasing in $|\xi|$. Now the case $m^{\prime}=0$ and examples as $a(\xi)=(\log \langle\xi\rangle)^{b}$, with some positive $b>0$, are also included.

Lemma 3.1.17. For a function $a \in \mathcal{A}$ let

$$
N_{j}^{a}=\sup \left\{|\xi|: a(\xi) \leqslant 2^{j}\right\}, \quad \text { for any } j \in \mathbb{N}_{0} .
$$

The sequence $N^{a}=\left(N_{j}^{a}\right)_{j \in \mathbb{N}_{0}}$ is a strongly increasing sequence in the sense of Definition 2.2.1.

Proof. It is clear from (3.16) that $N^{a}=\left(N_{j}^{a}\right)_{j \in \mathbb{N}_{0}}$ is increasing. Let us sketch the proof of the existence of a constant $\kappa_{0} \in \mathbb{N}$ such that $2 N_{j}^{a} \leqslant N_{k}^{a}$, for any $j$ and $k$ such that $j+\kappa_{0} \leqslant k$.

For simplicity let us denote $N_{j}^{a}=N_{j}$, for any $j \in \mathbb{N}$. From the definition of the numbers $N_{j}$ it follows that there exists an $\xi_{0}$ with

$$
\frac{N_{j}}{2} \leqslant\left|\xi_{0}\right| \leqslant N_{j} \quad \text { and } \quad a\left(\xi_{0}\right) \leqslant 2^{j} .
$$

Due to the properties of the function $a$ it is clear that the function $t \mapsto a\left(t \xi_{0}\right)$ is a one-dimensional continuous function with $\lim _{t \rightarrow \infty} a\left(t \xi_{0}\right)=\infty$. Consequently, for $\kappa_{0} \in \mathbb{N}$, there exists a $t_{0}>1$ with

$$
a\left(t_{0} \xi_{0}\right)=2^{j-\frac{1}{2}+\kappa_{0}} .
$$

Then taking $\eta_{0}=t_{0} \xi_{0}$ one has $\left|\eta_{0}\right|=t_{0}\left|\xi_{0}\right|>\left|\xi_{0}\right|$ and

$$
\left|\eta_{0}\right| \leqslant N_{j+\kappa_{0}}=\sup \left\{|\eta|: a(\eta) \leqslant 2^{j+\kappa_{0}}\right\} .
$$

Now applying property (iii) from Definition 3.1.11 we have

$$
\frac{2^{j}}{\left(N_{j} / 2\right)^{m}} \geqslant \frac{a\left(\xi_{0}\right)}{\left|\xi_{0}\right|^{m}} \geqslant \delta_{m} \frac{a\left(\eta_{0}\right)}{\left|\eta_{0}\right|^{m}} \geqslant \delta_{m} \frac{2^{j-\frac{1}{2}+\kappa_{0}}}{N_{j+\kappa_{0}}^{m}},
$$

for sufficiently large $j$ (depending on $R$ in property (iii)) and arbitrary $\kappa_{0} \in \mathbb{N}$.

Consequently,

$$
N_{j+\kappa_{0}} \geqslant \frac{1}{2}\left(\delta_{m} 2^{\kappa_{0}-\frac{1}{2}}\right)^{1 / m} N_{j}
$$

and using the fact that $\left(N_{j}\right)_{j \in \mathbb{N}}$ is increasing we have $N_{k} \geqslant N_{j+\kappa_{0}} \geqslant 2 N_{j}$ if $k \geqslant j+\kappa_{0}$, for a fixed large enough $\kappa_{0}$.

This completes the proof that $\left(N_{j}\right)_{j \in \mathbb{N}_{0}}$ is strongly increasing.

Remark 3.1.18. Given an admissible function $a \in \mathcal{A}$ we can define, for any $r>0$,

$$
N_{j}^{a, r}=\sup \left\{|\xi|: a(\xi) \leqslant 2^{j r}\right\}, \quad \text { for any } j \in \mathbb{N}_{0} .
$$

Using the same technique as above it is easy to see that $N^{a, r}=\left(N_{j}^{a, r}\right)_{j \in \mathbb{N}_{0}}$ is again a strongly increasing sequence. 
Lemma 3.1.19. If $a: \mathbb{R}^{n} \rightarrow \mathbb{R}$ satisfies (3.12) then for any real number $m$ the function $b(\xi)=(1+a(\xi))^{m}$ also satisfies (3.12).

Proof. Applying (3.15) with $f(t)=t^{m}$ and $1+a(\cdot)$ instead of $a(\cdot)$, and using the assumption on $a$, we get, for any $\alpha \in \mathbb{N}_{0}^{n}$,

$$
\begin{aligned}
\left|D^{\alpha} b(\xi)\right| \leqslant & \sum_{j=1}^{|\alpha|} c_{j}(1+a(\xi))^{m-j} \\
& \times \sum \frac{\alpha !}{\delta_{\beta} ! \delta_{\gamma} ! \cdot \ldots \cdot \delta_{\omega} !}\left|\frac{D^{\beta}(1+a(\xi))}{\beta !}\right|^{\delta_{\beta}} \cdot \ldots \cdot\left|\frac{D^{\omega}(1+a(\xi))}{\omega !}\right|^{\delta_{\omega}} \\
\leqslant & \sum_{j=1}^{|\alpha|} c_{j}(1+a(\xi))^{m-j} c_{\alpha} \prod_{\beta}(1+a(\xi))^{\delta_{\beta}}\langle\xi\rangle^{-\delta_{\beta}|\beta|} \\
\leqslant & c_{\alpha}^{\prime}(1+a(\xi))^{m}\langle\xi\rangle^{-|\alpha|} ;
\end{aligned}
$$

and this shows that $b$ also satisfies (3.12).

The next result generalises the Littlewood-Paley-type Theorem 3.1.5. Recall the notation $\sigma^{s}=\left(2^{j s}\right)_{j \in \mathbb{N}_{0}}$.

Theorem 3.1.20. Let $a \in \mathcal{A}$ be an admissible symbol, let $r>0$ and let $N=N^{a, r}$, the strongly increasing sequence associated to a and $r$, see (3.17). Let $1<p<\infty$ and $1<q<\infty$.

Then, for any real number $s$, we have

$$
\left\|(\mathrm{id}+a(D))^{s / r} u\left|F_{p, q}^{\sigma^{0}, N^{a, r}}\|\sim\| u\right| F_{p, q}^{\sigma^{s}, N^{a, r}}\right\|
$$

and the corresponding assertion for B-spaces.

Proof. We will give here the proof for $F$-spaces since the proof for $B$-spaces is essentially similar but simpler.

Using Lemma 3.1.19 and the construction of the strongly increasing sequence $N=\left(N_{j}^{a, r}\right)_{j \in \mathbb{N}_{0}}$, we get, for any multi-index $\alpha$,

$$
\begin{aligned}
D^{\alpha}\left(2^{-j s}(1+a(\xi))^{s / r} \chi_{\operatorname{supp} \varphi_{j}^{N, J}}(\xi)\right) & \leqslant c_{\alpha} 2^{-j s}(1+a(\xi))^{s / r}\langle\xi\rangle^{-\alpha} \chi_{\operatorname{supp} \varphi_{j}^{N, J}}(\xi) \\
& \leqslant c_{\alpha}^{\prime}\langle\xi\rangle^{-\alpha},
\end{aligned}
$$

since $(1+a(\xi))^{s / r} \sim 2^{j s}$ on $\operatorname{supp} \varphi_{j}^{N, J} \subset\left\{\xi \in \mathbb{R}^{n}: N_{j-J \kappa_{0}} \leqslant|\xi| \leqslant N_{j+J_{\kappa_{0}}}\right\}$ Consequently we may apply Proposition 3.1.1, diagonal case - see (3.2), and get

$$
\begin{aligned}
\left\|(\operatorname{id}+a(D))^{s / r} u \mid F_{p, q}^{\sigma^{0}, N}\right\| & =\left\|\mathcal{F}^{-1}\left[\varphi_{j}^{N, J}(\xi)(1+a(\xi))^{s / r} \mathcal{F} u\right] \mid L_{p}\left(l_{q}\right)\right\| \\
& =\left\|\mathcal{F}^{-1}\left[2^{-j s}(1+a(\xi))^{s / r} 2^{j s} \varphi_{j}^{N, J}(\xi) \mathcal{F} u\right] \mid L_{p}\left(l_{q}\right)\right\| \\
& \leqslant c\left\|2^{j s} \varphi_{j}^{N, J}(D) u \mid L_{p}\left(l_{q}\right)\right\| \\
& =c\left\|u \mid F_{p, q}^{\sigma^{s}, N}\right\| .
\end{aligned}
$$


For the reverse inequality note that

$$
\begin{aligned}
D^{\alpha}\left(2^{j s}(1+a(\xi))^{-s / r} \chi_{\operatorname{supp} \varphi_{j}^{N, J}}(\xi)\right) & \leqslant c_{\alpha} 2^{j s}(1+a(\xi))^{-s / r}\langle\xi\rangle^{-\alpha} \chi_{\operatorname{supp} \varphi_{j}^{N, J}}(\xi) \\
& \leqslant c_{\alpha}^{\prime}\langle\xi\rangle^{-\alpha}
\end{aligned}
$$

since $(1+a(\xi))^{-s / r} \sim 2^{-j s}$ on $\operatorname{supp} \varphi_{j}^{N, J} \subset\left\{\xi \in \mathbb{R}^{n}: N_{j-J \kappa_{0}} \leqslant|\xi| \leqslant N_{j+J \kappa_{0}}\right\}$. Consequently we may apply again Proposition 3.1.1, diagonal case - see (3.2), and get

$$
\begin{aligned}
\left\|u \mid F_{p, q}^{\sigma^{s}, N}\right\| & =\left\|2^{j s} \varphi_{j}^{N, J}(D) u \mid L_{p}\left(l_{q}\right)\right\| \\
& =\left\|\mathcal{F}^{-1}\left[2^{j s}(1+a(\xi))^{-s / r} \varphi_{j}^{N, J}(\xi)(1+a(\xi))^{s / r} \mathcal{F} u\right] \mid L_{p}\left(l_{q}\right)\right\| \\
& \leqslant c\left\|\mathcal{F}^{-1}\left[\varphi_{j}^{N, J}(\xi)(1+a(\xi))^{s / r} \mathcal{F} u\right] \mid L_{p}\left(l_{q}\right)\right\| \\
& =c\left\|(\mathrm{id}+a(D))^{s / r} u \mid F_{p, q}^{\sigma^{0}, N}\right\|,
\end{aligned}
$$

which completes the proof.

As a simple consequence of the above theorem and of Theorem 3.1.5 we get:

Corollary 3.1.21. Let $a \in \mathcal{A}$ be an admissible symbol, and let $N=N^{a, 2}$, the strongly increasing sequence associated to $a$ and to $r=2$, see (3.17). Let $1<p<\infty$.

Then, for any real number s, we have

$$
\left\|(\mathrm{id}+a(D))^{s / 2} u\left|L_{p}\|\sim\| u\right| F_{p, 2}^{\sigma^{s}, N^{a, 2}}\right\| .
$$

Remark 3.1.22. Note that if $s>0$ a similar result as stated in Corollary 3.1.21 was mentioned in [Ka79].

\subsection{The cases $0<p \leqslant 1$ and $p=\infty$}

To extend the definition of the spaces of generalised smoothness to $p=\infty, p=1$ and $0<p<1$, an additional assumption on the sequence $N$ is necessary. The reason is, that we can not use in these cases the previous Fourier-multiplier theorem (Proposition 3.1.1).

A substitute of it is a Fourier-multiplier theorem which was proved in spaces of entire analytic functions by the help of maximal functions.

Proposition 3.2.1. Let $0<p<\infty, 0<q \leqslant \infty$. For every $j \in \mathbb{N}_{0}$, let $R_{j}>0$ be a given number, let $\Omega_{j}=\left\{\xi \in \mathbb{R}^{n}:|\xi| \leqslant R_{j}\right\}$ and let $\Omega=\left(\Omega_{j}\right)_{j \in \mathbb{N}_{0}}$.

If $0<t<\min (p, q)$ then there exists a constant $c>0$ such that

$$
\left\|\left(\sup _{z \in \mathbb{R}^{n}} \frac{\left|f_{j}(\cdot-z)\right|}{1+\left|R_{j} z\right|^{n / t}}\right)_{j \in \mathbb{N}_{0}}\left|L_{p}\left(l_{q}\right)\|\leqslant c\| f\right| L_{p}\left(l_{q}\right)\right\|,
$$

for all $f=\left(f_{j}\right)_{j \in \mathbb{N}_{0}} \in L_{p}\left(l_{q}\right)$ such that $\operatorname{supp} \mathcal{F} f_{j} \subset \Omega_{j}$, for all $j \in \mathbb{N}_{0}$. 
This result was proved in [Tr83, Theorem 1.6.2] and was the main tool in the proof of the following Fourier-multiplier theorem [Tr83, Theorem 1.6.3]:

Proposition 3.2.2. Let $0<p<\infty, 0<q \leqslant \infty$. Let $\left(\Omega_{j}\right)_{j \in \mathbb{N}_{0}}$ be a sequence of compact subsets of $\mathbb{R}^{n}$ and $d_{j}>0$ be the diameter of $\Omega_{j}$.

If $t>n / 2+n / \min (p, q)$, then there exists a constant $c>0$ such that

$$
\left\|\left(M_{j}(D) f_{j}\right)_{j \in \mathbb{N}_{0}}\left|L_{p}\left(l_{q}\right)\left\|\leqslant c \sup _{j \in \mathbb{N}_{0}}\right\| M_{j}\left(d_{j} \cdot\right)\right| H_{2}^{t}\right\| \cdot\left\|\left(f_{j}\right)_{j \in \mathbb{N}_{0}} \mid L_{p}\left(l_{q}\right)\right\|
$$

holds for all systems $\left(f_{j}\right)_{j \in \mathbb{N}_{0}} \in L_{p}\left(l_{q}\right)$ with $\operatorname{supp} \mathcal{F} f_{j} \subset \Omega_{j}$ for all $j$, and all sequences $\left(M_{j}\right)_{j \in \mathbb{N}_{0}} \subset H_{2}^{t}$, where $H_{2}^{t}$ is the standard Bessel potential space of smoothness $t$.

Let $\left(\varphi_{j}^{N, J}\right)_{j \in \mathbb{N}_{0}}$ be a usual system associated to a strongly increasing sequence $N=\left(N_{j}\right)_{j \in \mathbb{N}_{0}}$. Then an easy computation shows that for an integer $L$ we have

$$
\left\|\varphi_{j}^{N, J}\left(2 N_{j+J \kappa_{0}} \cdot\right) \mid W_{2}^{L}\right\| \leqslant c\left(2 N_{j+J \kappa_{0}} N_{j-J \kappa_{0}}^{-1}\right)^{L}
$$

but unfortunately the right-hand side is - in general - not uniformly bounded with respect to $j$. This happens only if the sequence $\left(N_{j}\right)_{j \in \mathbb{N}_{0}}$ is additionally of bounded growth.

Assuming $N$ is of bounded growth with $N_{j+1} \leqslant \lambda_{1} N_{j}$ the right-hand side of (3.18) can be estimated for arbitrary $j$ by $c \lambda_{1}^{2 J \kappa_{0}}$.

With this preparation we extend the definition of the spaces $B_{p, q}^{\sigma, N}$ and $F_{p, q}^{\sigma, N}$ to all $0<p \leqslant \infty$ and $0<p<\infty$, respectively.

Definition 3.2.3. Let $\left(N_{j}\right)_{j \in \mathbb{N}_{0}}$ be a strongly increasing sequence and of bounded growth. Let $J \in \mathbb{N}$, let $\left(\varphi_{j}^{N, J}\right)_{j \in \mathbb{N}_{0}} \in \Phi^{N, J}$, and let $\left(\sigma_{j}\right)_{j \in \mathbb{N}_{0}}$ be an admissible sequence.

(i) Let $0<p \leqslant \infty$ and $0<q \leqslant \infty$. The Besov space of generalised smoothness is

$$
B_{p, q}^{\sigma, N}=\left\{f \in \mathcal{S}^{\prime}:\left\|f\left|B_{p, q}^{\sigma, N}\|=\|\left(\sigma_{j} \varphi_{j}^{N, J}(D) f\right)_{j \in \mathbb{N}_{0}}\right| l_{q}\left(L_{p}\right)\right\|<\infty\right\} .
$$

(ii) Let $0<p<\infty$ and $0<q \leqslant \infty$. The Triebel-Lizorkin space of generalised smoothness is

$$
F_{p, q}^{\sigma, N}=\left\{f \in \mathcal{s}^{\prime}:\left\|f\left|F_{p, q}^{\sigma, N}\|=\|\left(\sigma_{j} \varphi_{j}^{N, J}(D) f\right)_{j \in \mathbb{N}_{0}}\right| L_{p}\left(l_{q}\right)\right\|<\infty\right\} .
$$

Of course, it can be easily shown that all standard results (independence of the system $\left(\varphi_{j}\right)_{j \in \mathbb{N}_{0}}$, density of $\delta$, embeddings, lift-operator, etc.) extend to the whole scale of spaces considered in the above definition; compare [Tr83, Sect. 2.3] or [Tr86, Sect. 2.3].

In Section 4 we will consider strongly increasing sequences $N$ which are of bounded growth so we will be able to deal with all admissible parameters in Definition 3.2.3 of the spaces $B_{p, q}^{\sigma, N}$ and $F_{p, q}^{\sigma, N}$. 


\subsection{Examples and comparison with other classes}

As we have have already mentioned, if $N_{j}=2^{j}$ and $\sigma=\left(2^{j s}\right)_{j \in \mathbb{N}_{0}}$, then $B_{p, q}^{\sigma, N}$ and $F_{p, q}^{\sigma, N}$ are the classical Besov and Triebel-Lizorkin spaces $B_{p, q}^{s}$ and $F_{p, q}^{s}$.

It is the aim of this subsection to show that the function spaces considered so far in this work cover many other classes of function spaces of generalised smoothness known in the literature.

For simplicity we will restrict ourselves in this part to function spaces of Besov type. The scale of $F$-spaces is usually defined in most of the cases in a natural way but we will not go into details.

- In the middle of the seventies M.L. Goldman and G.A. Kalyabin introduced and investigated, independently, function spaces of generalised smoothness. These spaces are defined on the basis of expansions in series of entire functions, and are connected with a general covering method - see [Go79], [Ka79], [Ka80], [Go80] or [Go89].

Let $1<p<\infty, 1 \leqslant q \leqslant \infty$, let $\left(N_{j}\right)_{j \in \mathbb{N}_{0}}$ be strongly increasing, let $\left(\alpha_{j}\right)_{j \in \mathbb{N}_{0}}$ be of bounded growth and $\left(\alpha_{j}^{-1}\right)_{j \in \mathbb{N}_{0}} \in l_{q^{\prime}}$. Then let $\mathbf{B}_{p, q}^{\alpha, N}\left(\mathbb{R}^{n}\right)$ be the collection of all $f \in L_{p}$ such that

$$
f=\sum_{j=1}^{\infty} f_{j} \quad \text { in } \quad L_{p}
$$

with $\operatorname{supp}\left(\mathcal{F} f_{j}\right) \subset\left\{\xi \in \mathbb{R}^{n}:|\xi| \leqslant N_{j}\right\}$ and $\left\|\left(\alpha_{j} f_{j}\right)_{j \in \mathbb{N}_{0}} \mid l_{q}\left(L_{p}\right)\right\|<\infty$.

By a standardisation result $[\mathrm{Ka} 77 \mathrm{~b}], \mathbf{B}_{p, q}^{\alpha, N}\left(\mathbb{R}^{n}\right)$ can be identified with a space $\mathbf{B}_{p, q}^{\beta, M}\left(\mathbb{R}^{n}\right)$, where $\beta=\left(\beta_{j}\right)_{j \in \mathbb{N}_{0}}$ almost strongly increasing and of bounded growth (and therefore an admissible sequence), and where the sequence $M=\left(M_{j}\right)_{j \in \mathbb{N}_{0}}$ is determined by the sequences $\beta, \alpha$ and $N$ via

$$
M_{k}=N_{\kappa(k)} \quad \text { with } \quad \kappa(k)=\min \left\{m: \sum_{j=m}^{\infty} \alpha_{j}^{-q^{\prime}} \leqslant \beta_{k}^{-q^{\prime}}\right\} .
$$

A simple calculation shows that both $\left\|f \mid \mathbf{B}_{p, q}^{\alpha, N}\right\|$ and $\left\|f \mid \mathbf{B}_{p, q}^{\beta, M}\right\|$ are equivalent to

$$
\left\|f\left|B_{p, q}^{\beta, M}\|=\|\left(\beta_{k} \varphi_{k}^{M, K}(D) f\right)_{k \in \mathbb{N}_{0}}\right| l_{q}\left(L_{p}\right)\right\|,
$$

where $\left(\varphi_{k}^{M, K}\right)_{k \in \mathbb{N}_{0}}$ is a system from Section 2.3 associated to the covering defined by the sequence $\left(M_{k}\right)_{k \in \mathbb{N}_{0}}$ above. Consequently, the above spaces are a subclass of Besov spaces of generalised smoothness as introduced in Section 3.1.

Thus, in this way function spaces with 'positive' generalised smoothness, whose elements are at least $L_{p}$-functions, can be described.

Many results are known for the spaces $\mathbf{B}_{p, q}^{\alpha, N}$, for example, embedding theorems - see [Ka81], [Go80], [Go84b], [Go85] or [Go92]; trace theorems - see [Ka78], [Ka79], [Go79], [Go80]; and characterisations by differences and moduli of continuity - see [Go76], [Ka77b], [Ka80].

The last one leads to the following characterisation or definition, often used by M.L. Goldman. 
Let $\lambda:(0,1) \rightarrow \mathbb{R}^{+}$be a non-decreasing, continuous function with $\lim _{t \downarrow 0} \lambda(t)$ $=0, M \in \mathbb{N}$ and $1 \leqslant p, q \leqslant \infty$. Let

$$
B_{p, q}^{\lambda}\left(\mathbb{R}^{n}\right)=\left\{f \in L_{p}:\left(\int_{0}^{1}\left(\frac{\omega_{p}^{M}(f, t)}{\lambda(t)}\right)^{q} \frac{d \lambda(t)}{\lambda(t)}\right)^{1 / q}<\infty\right\},
$$

where

$$
\omega_{p}^{M}(f, t)=\sup _{|h|<t}\left\|\Delta_{h}^{M} u(\cdot) \mid L_{p}\right\|
$$

and $\Delta_{h}^{M}=\Delta_{h}^{1} \Delta_{h}^{M-1}$, where $\Delta_{h}^{1} u(x)=u(x+h)-u(x)$.

If, in addition, $t \mapsto \lambda(t) t^{-M}$ is increasing and $t \mapsto \lambda(t) t^{-\delta}$ is almost decreasing then

$$
B_{p, q}^{\lambda}\left(\mathbb{R}^{n}\right)=B_{p, q}^{\alpha, N}\left(\mathbb{R}^{n}\right),
$$

with $\alpha_{j}=2^{j}, N_{j}=h_{j}^{-1}, \lambda\left(h_{j}\right)=2^{-j} \lambda(1)$, compare [Go76], [KaLi87, Theorem 8.2] or, for a similar form, see [Ka80].

- In [Tr77, Chap. 2] a general covering method was also introduced and used to define and investigate general function spaces of Besov-Hardy-Sobolev type, $B_{p, q}^{s(x)}$ and $F_{p, q}^{s(x)}$ on $\mathbb{R}^{n}$. This approach was used also in [Go80]. It contains isotropic spaces, anisotropic spaces, spaces with dominating mixed derivatives and some others. In the case of the usual weight sequence $\left(2^{j}\right)_{j \in \mathbb{N}_{0}}$ all these special spaces were studied in detail in [ScTr87], [Tr83], [Tr92]. However the general approach was not developed further in its full generality.

- Other function spaces of generalised smoothness appear as a result of real interpolation with a function parameter - see [Me83] and [CoFe86]. In these papers, a function $\psi: \mathbb{R}^{+} \rightarrow \mathbb{R}^{+}$belongs to the class $\mathcal{B}$ if $\psi$ is continuous, $\psi(1)=1$ and, for all $t \in(0, \infty)$, holds

$$
\bar{\psi}(t)=\sup _{s>0} \frac{\psi(t s)}{\psi(s)}<\infty .
$$

Let $1<p, q<\infty$, let $\psi \in \mathscr{B}$ and let $\left(\varphi_{j}\right)_{j \in \mathbb{N}_{0}}$ the usual resolution of unity, associated to the symbol $|\xi|^{2}$ of the Laplacian and to the sequence $N_{j}^{|\cdot|^{2}, 2}=2^{j}$. Then

$$
B_{p, q}^{\psi}\left(\mathbb{R}^{n}\right)=\left\{u \in \boldsymbol{s}^{\prime}:\left(\sum_{j=0}^{\infty} \psi\left(2^{j}\right)^{q}\left\|\varphi_{j}(D) u \mid L_{p}\right\|^{q}\right)^{1 / q}<\infty\right\} .
$$

One has the following interpolation result:

$$
\left(L_{p}\left(\mathbb{R}^{n}\right), W_{p}^{k}\left(\mathbb{R}^{n}\right)\right)_{\rho_{1}, q}=B_{p, q}^{\rho}\left(\mathbb{R}^{n}\right) \text { where } \rho(t)=\left(\rho_{1}\left(t^{-k}\right)\right)^{-1} .
$$

Here $\psi(t)=t^{s} \log (1+t)^{b}$ with $s<0$ is now an admissible function too, related to the sequence $\alpha_{j}=2^{j s} j^{b}$ which does not fulfil $\left(\alpha_{j}^{-1}\right)_{j \in \mathbb{N}_{0}} \in l_{q^{\prime}}$. On the other hand, the decomposition is always fixed by the sequence $\left(N_{j}\right)_{j \in \mathbb{N}_{0}}=\left(2^{j}\right)_{j \in \mathbb{N}_{0}}$. 
With $\sigma_{j}=\psi\left(2^{j}\right)$, we have

$$
\bar{\psi}(1 / 2) \sigma_{j} \leqslant \sigma_{j+1} \leqslant \bar{\psi}(2) \sigma_{j},
$$

which means $\left(\sigma_{j}\right)_{j \in \mathbb{N}_{0}}$ is an admissible sequence and consequently these spaces are covered by Definition 3.1.2.

- Generalising fractal $d$-sets to $(d, \Psi)$-sets, D.E. Edmunds and H. Triebel considered in [EdTr98] and [EdTr99] function spaces of generalised smoothness, related to Example 2.2.5.

Let $\Psi$ be a positive monotone function on the interval $(0,1]$ such that there are some positive constants $b_{0}$ and $b_{1}$ with $b_{0} \Psi\left(2^{-j}\right) \leqslant \Psi\left(2^{-2 j}\right) \leqslant b_{1} \Psi\left(2^{-j}\right)$, for any $j \in \mathbb{N}_{0}$. Then

$$
B_{p, q}^{(s, \Psi)}=\left\{u \in \mathcal{S}^{\prime}:\left\|u \mid B_{p, q}^{(s, \Psi)}\right\|=\left(\sum_{j=0}^{\infty}\left(2^{j s} \Psi\left(2^{-j}\right)\right)^{q}\left\|\varphi_{j}(D) u \mid L_{p}\right\|^{q}\right)^{1 / q}<\infty\right\}
$$

(modification if $q=\infty$ ). Here $\left(\varphi_{j}\right)_{j \in \mathbb{N}_{0}}$ is again the usual resolution of unity associated to the sequence $N_{j}=2^{j}$.

Including the $F$-spaces an extensive study of these scales of spaces - embeddings, lifting properties, subatomic decompositions, local means, function spaces on fractals, entropy numbers and applications - was done by S. Moura in [Mo99] and [Mo01].

Again $\sigma_{j}=2^{j s} \Psi\left(2^{-j}\right)$ is an admissible sequence and $N_{j}=2^{j}$ is strongly increasing and of bounded growth. So, for all admissible parameters $p, q$, these spaces are covered by Definition 3.2.3, too.

- In [OpTr00] generalised smoothness of 'logarithmic' order was used to describe general embeddings of Pohozhaev-Trudinger type. The spaces under consideration in [OpTrO0] are defined as

$$
H^{\sigma, \alpha}\left(L_{p}\right)\left(\mathbb{R}^{n}\right)=\left\{u: u \in L_{p} \quad \text { and } \quad u=g_{\sigma, \alpha} * f, f \in L_{p}\right\},
$$

with $\mathcal{F} g_{\sigma, \alpha}(\xi)=\left(1+|\xi|^{2}\right)^{-\sigma / 2}\left(1+\log \left(1+|\xi|^{2}\right)\right)^{-\alpha}, \sigma \geqslant 0, \alpha$ real.

Similarly they defined spaces $H^{\sigma, \alpha}\left(L_{p, r}(\log L)^{\beta}\right)\left(\mathbb{R}^{n}\right)$, where $L_{p}$ is replaced by some suitable Lorentz-Zygmund space.

The first case is again covered by our definition, compare Theorem 3.1.20 and Corollary 3.1.21 with $a(\xi)=\mathcal{F} g_{\sigma, \alpha}(\xi)$.

- Motivated by the problem of constructing Markov processes starting in every point from $\mathbb{R}^{n}$ in [FJS01a] and [FJS01b], Bessel potential spaces $H_{p}^{\psi, s}\left(\mathbb{R}^{n}\right)$ associated to a continuous negative definite function $\psi: \mathbb{R}^{n} \rightarrow \mathbb{R}$ were introduced and studied (embeddings, interpolation, etc). Note that all examples of admissible functions from Example 3.1.15 are continuous negative definite functions.

For $1<p<\infty$ and $s \geqslant 0$ the space $H_{p}^{\psi, s}\left(\mathbb{R}^{n}\right)$ is the collection of all $f \in L_{p}$ such that

$$
\left\|f\left|H_{p}^{\psi, s}\left(\mathbb{R}^{n}\right)\|=\|(\mathrm{id}+\psi(D))^{\frac{s}{2}} f\right| L_{p}\right\|<\infty,
$$


whereas if $s<0$ the space $H_{p}^{\psi, s}\left(\mathbb{R}^{n}\right)$ is the closure of test functions in the above norm.

We want to point out here that the spaces $H_{p}^{\psi, s}\left(\mathbb{R}^{n}\right)$ can be regarded, at least for a class of continuous negative definite functions $\psi$, as function spaces of generalised smoothness of type $F_{p, q}^{\sigma^{s}, N}$. Indeed, considering the continuous negative definite function $\psi: \mathbb{R}^{n} \rightarrow \mathbb{R}$ of the form $\psi(\xi)=f\left(1+|\xi|^{2}\right)$ or $\psi(\xi)=f\left(|\xi|^{2}\right)$, respectively, where $f$ is a Bernstein function with $\lim _{t \rightarrow \infty} f(t)=\infty$, then $\psi$ is an admissible symbol in the sense of Definition 3.1.11 and we have only to apply Corollary 3.1.21 to see that

$$
H_{p}^{\psi, s}=F_{p, 2}^{\sigma^{s}, N^{\psi, 2}}
$$

\section{Local means and atomic decompositions}

\subsection{Preliminaries}

Assumption 4.1.1. Throughout the whole section we will assume $N=\left(N_{j}\right)_{j \in \mathbb{N}_{0}}$ is a sequence of real positive numbers such that there exist two numbers $1<\lambda_{0} \leqslant \lambda_{1}$ with

$$
\lambda_{0} N_{j} \leqslant N_{j+1} \leqslant \lambda_{1} N_{j}, \quad \text { for any } j \in \mathbb{N}_{0} .
$$

In particular $N$ is strongly increasing and of bounded growth.

Note that the first inequality in (4.1) implies in particular $N_{0}<N_{1}<N_{2} \ldots$. However we would like to point out that the condition $\lambda_{0}>1$ is a stronger restriction and it plays a key role in all the following considerations.

Remark 4.1.2. Nevertheless, the assumption concerning $\lambda_{0}$ is not restrictive with regard to the function spaces we are interested in. Indeed, let $\left(M_{j}\right)_{j \in \mathbb{N}_{0}}$ be strongly increasing and of bounded growth and let $\left(\beta_{j}\right)_{j \in \mathbb{N}_{0}}$ be an admissible sequence. Defining

$$
N_{j}=M_{j \kappa_{0}} \quad \text { and } \quad \sigma_{j}=\beta_{j \kappa_{0}}
$$

it is easy to see that the sequence $\left(N_{j}\right)_{j \in \mathbb{N}_{0}}$ satisfies (4.1) with $\lambda_{0}=2$ and

$$
B_{p, q}^{\beta, M}=B_{p, q}^{\sigma, N} \quad \text { and } \quad F_{p, q}^{\beta, M}=F_{p, q}^{\sigma, N} .
$$

This observation is similar to that in [Ka88, Remark 1].

Assumption 4.1.3. To avoid technical complications we will assume

$$
N_{1} \geqslant \lambda_{1}
$$

We should note that there is no loss of generality in assuming (4.2). Indeed, since $\lambda_{0}>1$, there exists an $m \in \mathbb{N}$ such that $\lambda_{0}^{m} N_{0} \geqslant \lambda_{1}$. Let

$$
m_{1}=\min \left\{m \in \mathbb{N}: \lambda_{0}^{m} N_{0} \geqslant \lambda_{1}\right\}
$$

and so $N_{m_{1}} \geqslant \lambda_{0}^{m_{1}} N_{0} \geqslant \lambda_{1}$. If we would not have $N_{1} \geqslant \lambda_{1}$ then in all considerations below one has to replace $N_{1}$ with $N_{m_{1}}$. 
Assumption 4.1.4. We will always denote $\sigma=\left(\sigma_{j}\right)_{j \in \mathbb{N}_{0}}$ an admissible sequence, this means that there are two constants $0<d_{0} \leqslant d_{1}<\infty$ such that

$$
d_{0} \sigma_{j} \leqslant \sigma_{j+1} \leqslant d_{1} \sigma_{j} \text { for any } j \in \mathbb{N}_{0} .
$$

Under the above conditions on the sequences $N$ and $\sigma$, the aim of this section is to give equivalent quasi-norms for the spaces $B_{p, q}^{\sigma, N}(0<p, q \leqslant \infty)$ and $F_{p, q}^{\sigma, N}$ $(0<p<\infty, 0<q \leqslant \infty)$ in terms of maximal functions, local means, and atomic decompositions.

\subsection{Equivalent quasi-norms}

Let $N=\left(N_{j}\right)_{j \in \mathbb{N}_{0}}$ be an admissible sequence of positive numbers satisfying (4.1) with $\lambda_{0}>1$. Then there exists an natural number $l_{0}=l_{0}\left(\lambda_{0}, \lambda_{1}\right)$ with

$$
\lambda_{0}^{l_{0}}>\lambda_{1} .
$$

We choose an $l_{0}>1$ satisfying (4.4) and fix it from now on.

Let $\mu_{0}, \mu \in \delta$ be two positive functions on $\mathbb{R}^{n}$ such that

$$
\mu_{0}(\xi)=1 \quad \text { if } \quad|\xi| \leqslant N_{1} \quad \text { and } \quad \operatorname{supp} \mu_{0} \subset\left\{\xi \in \mathbb{R}^{n}:|\xi| \leqslant \lambda_{0} N_{1}\right\}
$$

and

$$
\mu(\xi)=1 \quad \text { if } \quad \frac{1}{\lambda_{1}} \leqslant|\xi| \leqslant \lambda_{1} \quad \text { and } \quad \operatorname{supp} \mu \subset\left\{\xi \in \mathbb{R}^{n}: \frac{1}{\lambda_{0}^{l_{0}}} \leqslant|\xi| \leqslant \lambda_{0}^{l_{0}}\right\} .
$$

For any $j \geqslant 1$ we define

$$
\mu_{j}(\xi)=\mu\left(N_{j}^{-1} \xi\right), \quad \xi \in \mathbb{R}^{n} .
$$

Remark 4.2.1. Using (4.1) it is easy to see that we have

$$
\operatorname{supp} \mu_{j} \subset\left\{\xi \in \mathbb{R}^{n}: N_{j-l_{0}} \leqslant|\xi| \leqslant N_{j+l_{0}}\right\} \text { for any } j \geqslant 1 .
$$

This shows that for each fixed $j_{0} \in \mathbb{N}$ the set supp $\mu_{j_{0}}$ has a non-empty intersection with at most $4 l_{0}+1$ different supports of the functions $\mu_{j}$.

Moreover, a simple computation shows that for any multi-index $\alpha$ there is a constant $c_{\alpha}$ (depending on $\mu$ but not on $j$ ) such that

$$
\left|D^{\alpha} \mu_{j}(\xi)\right| \leqslant c_{\alpha}\langle\xi\rangle^{-|\alpha|} \text { for any } \xi \in \mathbb{R}^{n} \text { and any } j \in \mathbb{N} .
$$

Note that the family $\left(\mu_{j}\right)_{j \in \mathbb{N}_{0}}$ does not - in general - satisfy a condition of type (2.10) - resolution of "unity". However, we have a counterpart of (2.10) which reads as follows:

$$
\mu_{0}(\xi)+\sum_{j=1}^{\infty} \mu_{j}(\xi) \geqslant 1 \quad \text { for any } \xi \in \mathbb{R}^{n}
$$

Indeed, the sum in (4.8) is finite and each function $\mu_{j}, j \in \mathbb{N}_{0}$, is positive. If now $|\xi| \leqslant N_{1}$ then $\mu_{0}(\xi)=1$; if there is a $j_{0} \geqslant 2$ such that $N_{j_{0}-1} \leqslant|\xi| \leqslant N_{j_{0}+1}$ then it follows that $\frac{1}{\lambda_{1}} N_{j_{0}} \leqslant|\xi| \leqslant \lambda_{1} N_{j_{0}}$ and this implies $\mu_{j_{0}}(\xi)=1$. 
Theorem 4.2.2. Under the above assumptions on the sequences $\left(N_{j}\right)_{j \in \mathbb{N}_{0}},\left(\sigma_{j}\right)_{j \in \mathbb{N}_{0}}$ and on the functions $\mu_{0}$ and $\mu$, we have:

(i) Let $0<p \leqslant \infty$ and $0<q \leqslant \infty$. Then $f \in{\delta^{\prime}}^{\prime}\left(\mathbb{R}^{n}\right)$ belongs to $B_{p, q}^{\sigma, N}$ if, and only if,

$$
\left\|f\left|B_{p, q}^{\sigma, N}\left\|_{\mu}=\right\| \mu_{0}(D) f\right| L_{p}\right\|+\left(\sum_{j=1}^{\infty} \sigma_{j}^{q}\left\|\mu_{j}(D) f \mid L_{p}\right\|^{q}\right)^{1 / q}<\infty
$$

(with the usual modification if $q=\infty$ ). Moreover, $\left\|\cdot \mid B_{p, q}^{\sigma, N}\right\|_{\mu}$ is an equivalent quasi-norm in $B_{p, q}^{\sigma, N}$.

(ii) Let $0<p<\infty$ and $0<q \leqslant \infty$. Then $f \in{\delta^{\prime}}^{\prime}\left(\mathbb{R}^{n}\right)$ belongs to $F_{p, q}^{\sigma, N}$ if, and only if,

$$
\left\|f\left|F_{p, q}^{\sigma, N}\left\|_{\mu}=\right\| \mu_{0}(D) f\right| L_{p}\right\|+\left\|\left(\sum_{j=1}^{\infty} \sigma_{j}^{q}\left|\mu_{j}(D) f(\cdot)\right|^{q}\right)^{1 / q} \mid L_{p}\right\|<\infty
$$

(with the usual modification if $q=\infty$ ). Moreover, $\left\|\cdot \mid F_{p, q}^{\sigma, N}\right\|_{\mu}$ is an equivalent quasi-norm in $F_{p, q}^{\sigma, N}$.

Proof. We will indicate the proof in the more complicated case of $F$-spaces. To do this we will apply Proposition 3.2.2.

Let $\left(\varphi_{j}^{N}\right)_{j \in \mathbb{N}_{0}}$ be a smooth partition of unity satisfying (2.7)-(2.10), with $c_{\varphi}=1$, and let $\left\|f \mid F_{p, q}^{\sigma, N}\right\|_{\varphi}$ be the quasi-norm from Definition 3.2.3.

Choose $t>\frac{n}{2}+\frac{n}{\min (p, q)}$, an integer. Since for any $j \in \mathbb{N}_{0}$ clearly $\mu_{j}(\xi)=1$ on $\operatorname{supp} \varphi_{j}^{N}$ we get, applying Proposition 3.2.2,

$$
\begin{aligned}
\left\|f \mid F_{p, q}^{\sigma, N}\right\|_{\varphi} & =\left\|\sigma_{j} \mathcal{F}^{-1}\left[\varphi_{j}^{N} \mu_{j} \mathcal{F} f\right] \mid L_{p}\left(l_{q}\right)\right\| \\
& =\left\|\mathcal{F}^{-1} \varphi_{j}^{N} \mathcal{F}\left(\sigma_{j} \mathcal{F}^{-1}\left[\mu_{j} \mathcal{F} f\right]\right) \mid L_{p}\left(l_{q}\right)\right\| \\
& \leqslant c \sup _{j \in \mathbb{N}_{0}}\left\|\varphi_{j}^{N}\left(2 N_{j+l_{0}} \cdot\right)\left|H_{2}^{t}\|\cdot\| f\right| F_{p, q}^{\sigma, N}\right\|_{\mu} \\
& \leqslant c^{\prime} \cdot\left\|f \mid F_{p, q}^{\sigma, N}\right\|_{\mu},
\end{aligned}
$$

where $\left\|f \mid F_{p, q}^{\sigma, N}\right\|_{\mu}$ is the quasi-norm from (4.9) and we have used the fact that for any $\alpha$ with $|\alpha| \leqslant t$ there exists a constant $c_{\alpha}>0$ with

$$
\left\|D^{\alpha} \varphi_{j}^{N}\left(N_{j+1} \cdot\right) \mid L_{2}\right\| \leqslant c_{\alpha} \quad \text { for any } j \in \mathbb{N}_{0},
$$

as a simple consequence of properties (2.8) and (2.9).

To prove the reverse inequality we note that, due to the support properties of the functions $\mu_{j}$, we have, for any $j \in \mathbb{N}_{0}$,

$$
\mu_{j}=\sum_{k=-2 l_{0}}^{2 l_{0}} \mu_{j} \varphi_{j+k}^{N}
$$


where $\varphi_{-2 l_{0}}^{N}=\varphi_{-2 l_{0}+1}^{N}=\cdots=\varphi_{-1}^{N}=0$. Then again one has to apply Proposition 3.2.2 and to make appropriate changes in (4.10).

Consequently, $\left\|f \mid F_{p, q}^{\sigma, N}\right\|_{\varphi}$ and $\left\|f \mid F_{p, q}^{\sigma, N}\right\|_{\mu}$ are equivalent.

\subsection{Maximal functions and local means}

4.3.1. Some preparatory results. Before stating the main result of this subsection, see Theorem 4.3.4 below, we have to give some auxiliary results.

For any smooth function $\mu$ and for any $t>0$ we will use the notation

$$
\mu_{t}(x)=t^{-n} \mu\left(t^{-1} x\right) .
$$

The next lemma will play a key role in our further considerations.

Lemma 4.3.1. Let $M \geqslant-1$ be an integer and

$$
\varsigma_{M}=\left\{\mu \in \delta: D^{\alpha} \widehat{\mu}(0)=0 \text { for any }|\alpha| \leqslant M\right\} .
$$

For any $L>0$ there exists a constant $C_{L}>0$ such that

$$
\begin{aligned}
\sup _{z \in \mathbb{R}^{n}}\left|\left(\mu_{t} * \eta\right)(z)\right|(1+|z|)^{L} \leqslant & C_{L} \cdot t^{M+1} \cdot \max _{M+1 \leqslant|\beta| \leqslant \max (M+1, L+1)}\left\|D^{\beta} \widehat{\mu} \mid L_{\infty}\right\| . \\
& \cdot \max _{|\gamma| \leqslant L+1} \int_{\mathbb{R}^{n}}(1+|\xi|)^{M+1}\left|D^{\gamma} \widehat{\eta}(\xi)\right| d \xi,
\end{aligned}
$$

for any $t \in(0,1]$, for any $\mu \in \varsigma_{M}$ and any $\eta \in \&$.

Proof. By elementary properties of the Fourier transform it is easy to show that, for any $L>0$, there exists a constant $c_{L}$ such that, for any $g \in \delta$,

$$
\sup _{z \in \mathbb{R}^{n}}|g(z)|(1+|z|)^{L} \leqslant c_{L} \cdot \max _{|\alpha| \leqslant L+1}\left\|D^{\alpha} \widehat{g} \mid L_{1}\right\| .
$$

Taking $t \in(0,1], \mu \in \delta_{M}$ and $\eta \in \delta$ and inserting $g=\mu_{t} * \eta$ in (4.13) we have, in particular,

$$
\sup _{z \in \mathbb{R}^{n}}\left|\left(\mu_{t} * \eta\right)(z)\right| \leqslant c_{L} \cdot \max _{|\alpha| \leqslant L+1}\left\|D^{\alpha} \widehat{\mu_{t} * \eta} \mid L_{1}\right\| .
$$

Applying Leibniz's product rule for differentiation we have

$$
\begin{aligned}
\left|D^{\alpha}\left[\widehat{\mu_{t} * \eta}(\xi)\right]\right| & \leqslant c_{\alpha} \sum_{|\delta|+|\gamma|=|\alpha|}\left|D^{\delta}[\widehat{\mu}(t \xi)]\right| \cdot\left|D^{\gamma} \widehat{\eta}(\xi)\right| \\
& =c_{\alpha} \sum_{|\delta|+|\gamma|=\alpha} t^{|\delta|} \cdot\left|\left(D^{\delta} \widehat{\mu}\right)(t \xi)\right| \cdot\left|D^{\gamma} \widehat{\eta}(\xi)\right| .
\end{aligned}
$$

Fix now $\delta \leqslant \alpha$. Recall $D^{\alpha} \widehat{\mu}(0)=0$ for any $|\alpha| \leqslant M$. Then for any $\delta$ with $|\delta| \leqslant M$ we have, by Taylor's expansion theorem (with some positive constant $c_{\delta}$ ),

$$
\left|\left(D^{\delta} \widehat{\mu}\right)(t \xi)\right| \leqslant c_{\delta} \max _{|\beta|=M+1}\left\|D^{\beta} \widehat{\mu} \mid L_{\infty}\right\| \cdot(t|\xi|)^{M-|\delta|+1}
$$


and so

$$
\begin{array}{r}
t^{|\delta|} \cdot\left|\left(D^{\delta} \widehat{\mu}\right)(t \xi)\right| \leqslant c_{\delta} t^{M+1}\left(\max _{|\beta|=M+1}\left\|D^{\beta} \widehat{\mu} \mid L_{\infty}\right\|\right) \cdot(1+|\xi|)^{M+1} \\
\text { for any }|\delta| \leqslant M .
\end{array}
$$

We have now to distinguish if $M \geqslant L$ or not.

If $M \geqslant L$ then clearly the desired estimate (4.12) is a simple consequence of (4.14) using (4.15) and (4.16).

If $M<L$ then for a multi-index $\delta \leqslant \alpha$ it might happen that $M+1 \leqslant|\delta| \leqslant L+1$. Then for any such $\delta$ we have (recall $0<t \leqslant 1$ )

$$
t^{|\delta|} \cdot\left|\left(D^{\delta} \widehat{\mu}\right)(t \xi)\right| \leqslant c_{\delta} \cdot t^{M+1} \cdot \max _{M+1 \leqslant|\beta| \leqslant L+1}\left\|D^{\beta} \widehat{\mu} \mid L_{\infty}\right\| .
$$

Using (4.16) and (4.17) in (4.15), the inequality (4.12) follows again from (4.14).

Another result which we will use is the following:

Lemma 4.3.2. Let $0<p, q \leqslant \infty, \rho>0$. For any sequence $\left(g_{j}\right)_{j \in \mathbb{N}_{0}}$ of nonnegative measurable functions, denote

$$
G_{j}(x)=\sum_{m=0}^{\infty} 2^{-|j-m| \rho} g_{m}(x), \quad x \in \mathbb{R}^{n} .
$$

Then there exist some positive constants $c_{1}=c(q, \varrho)$ and $c_{2}=c_{2}(p, q, \varrho)$ such that

$$
\left\|\left(G_{j}\right)_{j \in \mathbb{N}_{0}}\left|L_{p}\left(l_{q}\right)\left\|\leqslant c_{1}\right\|\left(g_{j}\right)_{j \in \mathbb{N}_{0}}\right| L_{p}\left(l_{q}\right)\right\|
$$

and

$$
\left\|\left(G_{j}\right)_{j \in \mathbb{N}_{0}}\left|l_{q}\left(L_{p}\right)\left\|\leqslant c_{2}\right\|\left(g_{j}\right)_{j \in \mathbb{N}_{0}}\right| l_{q}\left(L_{p}\right)\right\| .
$$

The above lemma is well known and widely used. A proof can be found, for example, in [Ry99, Lemma 2]. We do not go into further details. need:

Let again $\left(N_{j}\right)_{j \in \mathbb{N}_{0}}$ be a sequence satisfying (4.1) with $\lambda_{0}>1$. We will also

Lemma 4.3.3. Let $0<\varrho \leqslant 1$ and $\left(b_{j}\right)_{j \in \mathbb{N}_{0}},\left(a_{j}\right)_{j \in \mathbb{N}_{0}}$ be two sequences taking values in $(0, \infty)$, respectively $(0, \infty)$. Assume that, for some $A_{0}>0$,

$$
\lim _{j \rightarrow \infty} a_{j} N_{j}^{-A_{0}} \quad \text { exists in } \mathbb{R}
$$

and that, for any $A>0$, there is a positive constant $C_{A}$ such that

$$
a_{j} \leqslant C_{A} \sum_{l=j}^{\infty}\left(N_{j} N_{l}^{-1}\right)^{A} b_{l} a_{l}^{1-\varrho}, \quad j \in \mathbb{N}_{0} .
$$

Then, for any $A>0$, we have

$$
a_{j}^{\varrho} \leqslant C_{A} \sum_{l=j}^{\infty}\left(N_{j} N_{l}^{-1}\right)^{A \varrho} b_{l}, \quad j \in \mathbb{N}_{0},
$$

with the same constant $C_{A}$. 
Proof. For any $j \in \mathbb{N}_{0}$ put $D_{j, A}=\sup _{m \geqslant j}\left(\left(N_{j} N_{m}^{-1}\right)^{A} a_{m}\right)$. By (4.19) we have

$$
\begin{aligned}
D_{j, A} & \leqslant \sup _{m \geqslant j}\left(\left(N_{j} N_{m}^{-1}\right)^{A} \cdot C_{A} \cdot \sum_{l=m}^{\infty}\left(N_{m} N_{l}^{-1}\right)^{A} b_{l} a_{l}^{1-\varrho}\right) \\
& \leqslant C_{A} \cdot \sum_{l=j}^{\infty}\left(N_{j} N_{l}^{-1}\right)^{A} b_{l} a_{l}^{1-\varrho} \leqslant C_{A} \cdot \sum_{l=j}^{\infty}\left(N_{j} N_{l}^{-1}\right)^{A \varrho} b_{l}\left(D_{j, A}\right)^{1-\varrho} .
\end{aligned}
$$

Consequently

$$
a_{j}^{\varrho} \leqslant\left(D_{j, A}\right)^{\varrho} \leqslant C_{A} \cdot \sum_{l=j}^{\infty}\left(N_{j} N_{l}^{-1}\right)^{A \varrho} b_{l},
$$

provided that $D_{j, A}$ is finite, which is satisfied by (4.18) at least for $A \geqslant A_{0}$. Thus we have proved (4.20) for $A \geqslant A_{0}$ and therefore also for $A<A_{0}$ with constant $C_{A_{0}}$ since the right-hand side of (4.20) decreases as $A$ increases.

Now let $A<A_{0}$ and assume that the right-hand side of (4.20) is finite (otherwise there is nothing to prove). By (4.20) with constant $C_{A_{0}}$, for $m \geqslant j$,

$$
\begin{aligned}
\left(N_{j} N_{m}^{-1}\right)^{A} a_{m} & \leqslant\left(N_{j} N_{m}^{-1}\right)^{A} \cdot C_{A_{0}}^{1 / \varrho}\left(\sum_{l=m}^{\infty}\left(N_{m} N_{l}^{-1}\right)^{A \varrho} b_{l}\right)^{1 / \varrho} \\
& \leqslant C_{A_{0}}^{1 / \varrho}\left(\sum_{l=m}^{\infty}\left(N_{j} N_{m}^{-1}\right)^{A \varrho} b_{l}\right)^{1 / \varrho}
\end{aligned}
$$

hence $D_{j, A}<\infty$, and we can use (4.21) which gives the desired estimate with constant $C_{A}$.

4.3.2. The theorem: equivalent quasi-norms based on maximal functions and local means. We are now prepared for the main result of this subsection.

Let $k_{0}$ and $k \in \&$, let $K \geqslant-1$ be an integer such that

$$
\begin{gathered}
\left|\widehat{k_{0}}(\xi)\right|>0 \text { for }|\xi| \leqslant N_{1}, \\
|\widehat{k}(\xi)|>0 \text { for } \frac{1}{\lambda_{1}} \leqslant|\xi| \leqslant \lambda_{1},
\end{gathered}
$$

and

$$
\int_{\mathbb{R}^{n}} x^{\alpha} k(x) d x=0 \text { for any }|\alpha| \leqslant K .
$$

Here (4.22) and (4.23) are Tauberian conditions, while (4.24) (which is in fact $D^{\alpha} \widehat{k}(0)=0$ for any $\left.|\alpha| \leqslant K\right)$ are moment conditions on $k$.

For any $r>0, f \in \varsigma^{\prime}$, and any $x \in \mathbb{R}^{n}$, let

$$
\left(k_{0}^{*} f\right)_{r}(x)=\sup _{z \in \mathbb{R}^{n}} \frac{\left|\left(k_{0} * f\right)(z)\right|}{(1+|x-z|)^{r}},
$$


and for $j \geqslant 1$

$$
\left(k_{N_{j}^{-1}}^{*} f\right)_{r}(x)=\sup _{z \in \mathbb{R}^{n}} \frac{\left|\left(k_{N_{j}^{-1}} * f\right)(z)\right|}{\left(1+N_{j}|x-z|\right)^{r}}
$$

(J. Peetre's maximal functions); we recall the notation $k_{N_{j}^{-1}}(x)=N_{j}^{n} k\left(N_{j} x\right)-$ see (4.11).

Theorem 4.3.4. Let $\left(N_{j}\right)_{j \in \mathbb{N}_{0}}$ be an admissible sequence with $\lambda_{0}>1$ and $\left(\sigma_{j}\right)_{j \in \mathbb{N}_{0}}$ be an admissible sequence.

Let

$$
K>-1+\frac{\log _{2} d_{1}}{\log _{2} \lambda_{0}}
$$

and let $k_{0}$ and $k$ functions from $\&$ which satisfy conditions (4.22)-(4.24)from above.

Let $0<p<\infty$, respectively $0<p \leqslant \infty$, let $0<q \leqslant \infty$, and let $r>\frac{n}{\min (p, q)}$, respectively $r>\frac{n}{p}$.

Then there exist two constants $c, c^{\prime}>0$ such that, for all $f \in \varsigma^{\prime}$,

$$
\left\|\left(k_{0}^{*} f\right)_{r}\left|L_{p}\|+\|\left(\sigma_{j}\left(k_{N_{j}^{-1}}^{*} f\right)_{r}\right)_{j \in \mathbb{N}}\right| L_{p}\left(l_{q}\right)\right\| \leqslant c\left\|f \mid F_{p, q}^{\sigma, N}\right\|
$$

and

$$
\left\|f \mid F_{p, q}^{\sigma, N}\right\| \leqslant c^{\prime}\left(\|\left(k_{0} * f\left|L_{p}\|+\|\left(\sigma_{j}\left(k_{N_{j}^{-1}} * f\right)\right)_{j \in \mathbb{N}}\right| L_{p}\left(l_{q}\right) \|\right),\right.
$$

respectively

$$
\left\|\left(k_{0}^{*} f\right)_{r}\left|L_{p}\|+\|\left(\sigma_{j}\left(k_{N_{j}^{-1}}^{*} f\right)_{r}\right)_{j \in \mathbb{N}}\right| l_{q}\left(L_{p}\right)\right\| \leqslant c\left\|f \mid B_{p, q}^{\sigma, N}\right\|
$$

and

$$
\left\|f \mid B_{p, q}^{\sigma, N}\right\| \leqslant c^{\prime}\left(\|\left(k_{0} * f\left|L_{p}\|+\|\left(\sigma_{j}\left(k_{N_{j}^{-1}} * f\right)\right)_{j \in \mathbb{N}}\right| l_{q}\left(L_{p}\right) \|\right) .\right.
$$

Remark 4.3.5. Note that the above inequalities are valid for all $f \in \delta^{\prime}$.

It is easy to see that, for any $x \in \mathbb{R}^{n}$ and any $f \in \mathcal{s}^{\prime}$, we have $\left|\left(k_{N_{j}^{-1}} * f\right)(x)\right| \leqslant$ $\left(k_{N_{j}^{-1}}^{*} f\right)_{r}(x)$. This shows that the right-hand side in (4.29) is less than the left-hand side in (4.28).

Consequently the left-hand side in (4.28) and the right-hand side in (4.29) are equivalent quasi-norms in $F_{p, q}^{\sigma, N}$.

Of course a corresponding assertion is valid for the spaces $B_{p, q}^{\sigma, N}$, now based on (4.30) and (4.31). 
4.3.3. Proof of Theorem 4.3.4. We will present here the proof of the inequalities (4.28) and (4.29). The inequalities (4.30) and (4.31) can be proved in a similar manner interchanging the roles of the quasi-norms in $L_{p}$ and $l_{q}$.

Step 1 . Take any pair of functions $\theta_{0}$ and $\theta \in \delta$ such that

$$
\left|\widehat{\theta_{0}}(\xi)\right|>0 \quad \text { if } \quad|\xi| \leqslant N_{1}
$$

and

$$
\widehat{\theta}(\xi) \mid \geqslant C>0 \quad \text { if } \quad \frac{1}{\lambda_{1}} \leqslant|\xi| \leqslant \lambda_{1},
$$

and define, for any $r>0$, the functions $\left(\theta_{0}^{*} f\right)_{r}$ and $\left(\theta_{N_{j}^{-1}}^{*} f\right)_{r}$ as in (4.25) and (4.26), where $\theta_{N_{j}^{-1}}(x)=N_{j}^{n} \theta\left(N_{j} x\right)$.

We will prove in this step that there is a constant $c>0$ such that, for any $f \in \delta^{\prime}$,

$$
\begin{gathered}
\left\|\left(k_{0}^{*} f\right)_{r}\left|L_{p}\|+\|\left(\sigma_{j}\left(k_{N_{j}^{-1}}^{*} f\right)_{r}\right)_{j \in \mathbb{N}}\right| L_{p}\left(l_{q}\right)\right\| \\
\leqslant c\left(\left\|\left(\theta_{0}^{*} f\right)_{r}\left|L_{p}\|+\|\left(\sigma_{j}\left(\theta_{N_{j}^{-1}}^{*} f\right)_{r}\right)_{j \in \mathbb{N}}\right| L_{p}\left(l_{q}\right)\right\|\right) .
\end{gathered}
$$

Take $\left(\varphi_{j}^{N}\right)_{j \in \mathbb{N}_{0}}$, a fixed partition of unity associated to $\left(N_{j}\right)_{j \in \mathbb{N}_{0}}$, that means $\left(\varphi_{j}^{N}\right)_{j \in \mathbb{N}_{0}}$ has the properties (2.7)-(2.10) with $c_{\varphi}=1$.

We define the functions $\psi_{j} \in C_{0}^{\infty}\left(\mathbb{R}^{n}\right), j \in \mathbb{N}_{0}$, by

$$
\widehat{\psi_{0}}(\xi)=\frac{\varphi_{0}^{N}(\xi)}{\widehat{\theta_{0}}(\xi)} \quad \text { and } \quad \widehat{\psi_{j}}(\xi)=\frac{\varphi_{j}^{N}(\xi)}{\widehat{\theta}\left(N_{j}^{-1} \xi\right)} \quad \text { for } \quad j \in \mathbb{N} \text {. }
$$

Due to the properties of the functions $\theta_{0}$ and $\theta$, the functions $\widehat{\psi_{0}}$ and $\widehat{\psi_{j}}$ are well defined and it is easy to see that, for any $j \in \mathbb{N}$, we have supp $\widehat{\psi_{j}} \subset\left\{\xi \in \mathbb{R}^{n}\right.$ : $\left.N_{j-1} \leqslant|\xi| \leqslant N_{j+1}\right\}$.

Moreover, applying the rule of differentiation for a product of functions, using (2.9) and (4.32) it follows that for any multi-index $\alpha$ there is a constant $c_{\alpha}>0$ such that, for any $j \geqslant 1$,

$$
\left|D^{\alpha} \widehat{\psi_{j}}(\xi)\right| \leqslant c_{\alpha}\langle\xi\rangle^{-|\alpha|} \text { for any } \xi \in \mathbb{R}^{n} .
$$

From (4.34) clearly

$$
1=\widehat{\theta_{0}}(\xi) \widehat{\psi_{0}}(\xi)+\sum_{j=1}^{\infty} \widehat{\psi_{j}}(\xi) \widehat{\theta}\left(N_{j}^{-1} \xi\right)
$$

and so, for any $f \in \varsigma^{\prime}$,

$$
f=\psi_{0} * \theta_{0} * f+\sum_{m=1}^{\infty} \psi_{m} * \theta_{N_{m}^{-1}} * f .
$$


Consequently, we have, for any $j \geqslant 1$,

$$
k_{N_{j}^{-1}} * f=k_{N_{j}^{-1}} * \psi_{0} * \theta_{0} * f+\sum_{m=1}^{\infty} k_{N_{j}^{-1}} * \psi_{m} * \theta_{N_{m}^{-1}} * f .
$$

For a fixed $m \geqslant 1$ one has

$$
\begin{aligned}
\mid\left(k_{N_{j}^{-1}} * \psi_{m}\right. & \left.* \theta_{N_{m}^{-1}} * f\right)(y) \mid \\
& \leqslant \int_{\mathbb{R}^{n}}\left|\left(k_{N_{j}^{-1}} * \psi_{m}\right)(z)\right| \cdot\left|\left(\theta_{N_{m}^{-1}} * f\right)(y-z)\right| d z \\
& \leqslant\left(\theta_{N_{m}^{-1}}^{*} f\right)_{r}(y) \cdot \int_{\mathbb{R}^{n}}\left|\left(k_{N_{j}^{-1}} * \psi_{m}\right)(z)\right| \cdot\left(1+N_{m}|z|\right)^{r} d z \\
& =\left(\theta_{N_{m}^{-1}}^{*} f\right)_{r}(y) \cdot I_{j m} .
\end{aligned}
$$

We are going now to obtain convenient estimates from above for the integral $I_{j m}$ in (4.37).

First, let $m \leqslant j$.

After a change of variables, inserting $k_{N_{j}^{-1}}(x)=N_{j}^{n} k\left(N_{j} x\right)$ we have

$$
\begin{aligned}
I_{j m} & =\int_{\mathbb{R}^{n}}\left|\left(k_{N_{j}^{-1}} * \psi_{m}\right)(z)\right| \cdot\left(1+N_{m}|z|\right)^{r} d z \\
& =N_{m}^{-n} \int_{\mathbb{R}^{n}}\left|\left(k_{N_{j}^{-1}} * \psi_{m}\right)\left(N_{m}^{-1} u\right)\right| \cdot(1+|u|)^{r} d u \\
& =N_{m}^{-n} \int_{\mathbb{R}^{n}}\left|\int_{\mathbb{R}^{n}} N_{j}^{n} N_{m}^{-n} k\left(N_{j} N_{m}^{-1} u-N_{j} N_{m}^{-1} v\right) \psi_{m}\left(N_{m}^{-1} v\right) d v\right| \cdot(1+|u|)^{r} d u \\
& =N_{m}^{-n} \int_{\mathbb{R}^{n}}\left|\left(k_{N_{j}^{-1} N_{m}} * \psi_{m}\left(N_{m}^{-1} \cdot\right)\right)(u)\right| \cdot(1+|u|)^{r} d u,
\end{aligned}
$$

where again $k_{t}(x)=t^{-n} k\left(t^{-1} x\right)$. It follows that for some positive constant $c$ (independent of $j$ and $m$ )

$$
I_{j m} \leqslant c N_{m}^{-n} \sup _{u \in \mathbb{R}^{n}}\left(\left|\left(k_{N_{j}^{-1} N_{m}} * \psi_{m}\left(N_{m}^{-1} \cdot\right)\right)(u)\right| \cdot(1+|u|)^{r+n+1}\right) .
$$

We may apply Lemma 4.3.1 with $t=N_{j}^{-1} N_{m} \leqslant 1, \mu=k \in \wp_{K}$ ( $k$ has $K$ moment conditions), $\eta=\psi_{m}\left(N_{m}^{-1} \cdot\right)$; taking $L=r+n+1$ we obtain (with some positive constant $c_{1}$ )

$$
\begin{aligned}
I_{j m} \leqslant c_{1} N_{m}^{-n}\left(N_{j}^{-1} N_{m}\right)^{K+1} \max _{K+1 \leqslant|\beta| \leqslant \max (K+1, r+n+2)}\left\|D^{\beta} \widehat{k} \mid L_{\infty}\right\| \cdot \\
\cdot \max _{|\alpha| \leqslant r+n+2} \int_{\mathbb{R}^{n}}(1+|\xi|)^{K+1}\left|D^{\alpha}\left[\psi_{m}\left(N_{m}^{-1} \cdot\right)\right](\xi)\right| d \xi \\
\leqslant c_{2} N_{m}^{-n}\left(N_{j}^{-1} N_{m}\right)^{K+1} \max _{|\alpha| \leqslant r+n+2} \int_{\mathbb{R}^{n}}(1+|\xi|)^{K+1}\left|D^{\alpha}\left[\widehat{\psi_{m}}\left(N_{m} \xi\right)\right]\right| N_{m}^{n} d \xi \\
=c_{2}\left(N_{j}^{-1} N_{m}\right)^{K+1} \max _{|\alpha| \leqslant r+n+2} \int_{\mathbb{R}^{n}}(1+|\xi|)^{K+1} N_{m}^{|\alpha|}\left|\left(D^{\alpha} \widehat{\psi_{m}}\right)\left(N_{m} \xi\right)\right| d \xi .
\end{aligned}
$$


Due to the localisation of the support of $\widehat{\psi_{m}}$ the last integral is in fact taken over the set $\left\{\xi \in \mathbb{R}^{n}: \frac{1}{\lambda_{1}} \leqslant|\xi| \leqslant \lambda_{1}\right\}$. Using (4.35) we get

$$
I_{j m} \leqslant c_{3}\left(N_{j}^{-1} N_{m}\right)^{K+1} \max _{|\alpha| \leqslant r+n+2} \int_{\frac{1}{\lambda_{1}} \leqslant|\xi| \leqslant \lambda_{1}}(1+|\xi|)^{K+1} N_{m}^{|\alpha|}\left(1+N_{m}|\xi|\right)^{-|\alpha|} d \xi,
$$

which is

$$
I_{j m} \leqslant c\left(N_{j}^{-1} N_{m}\right)^{K+1},
$$

with some positive constant $c>0$ independent of $j$ and $m$.

Let now $m>j$.

Then, again making use of changing of variables, and inserting $k_{N_{j}^{-1}}(x)=$ $N_{j}^{n} k\left(N_{j} x\right)$, we have

$$
\begin{aligned}
I_{j m} & =\int_{\mathbb{R}^{n}}\left|\left(k_{N_{j}^{-1}} * \psi_{m}\right)(z)\right|\left(1+N_{m}|z|\right)^{r} d z \\
& \leqslant\left(N_{j}^{-1} N_{m}\right)^{r} \int_{\mathbb{R}^{n}}\left|\left(k_{N_{j}^{-1}} * \psi_{m}\right)(z)\right|\left(1+N_{j}|z|\right)^{r} d z \\
& =\left(N_{j}^{-1} N_{m}\right)^{r} N_{j}^{-n} \int_{\mathbb{R}^{n}}\left|\int_{\mathbb{R}^{n}} k_{N_{j}^{-1}}\left(N_{j}^{-1} u-v\right) \psi_{m}(v) d v\right|(1+|u|)^{r} d u \\
& =\left(N_{j}^{-1} N_{m}\right)^{r} N_{j}^{-n} \int_{\mathbb{R}^{n}}\left|\int_{\mathbb{R}^{n}} \psi_{m}\left(N_{j}^{-1} y\right) k(u-y) d y\right|(1+|u|)^{r} d u \\
& =\left(N_{j}^{-1} N_{m}\right)^{r} N_{j}^{-n} \int_{\mathbb{R}^{n}}\left|\left(\psi_{m}\left(N_{j}^{-1} \cdot\right) * k\right)(u)\right|(1+|u|)^{r} d u .
\end{aligned}
$$

Consequently there exists a constant $c>0$, independent of $j$ and $m$, such that, for any $L \geqslant r+n+1$,

$$
I_{j m} \leqslant c\left(N_{j} N_{m}^{-1}\right)^{-r} N_{j}^{-n} \cdot \sup _{u \in \mathbb{R}^{n}}\left(\left|\left(\psi_{m}\left(N_{j}^{-1} \cdot\right) * k\right)(u)\right|(1+|u|)^{L}\right) .
$$

Again using Lemma 4.3.1 we define, for any $m \geqslant 1$, the function

$$
\psi^{(m)}(u)=\psi_{m}\left(N_{m}^{-1} u\right), \quad u \in \mathbb{R}^{n} .
$$

Then $\widehat{\psi^{(m)}}(\xi)=N_{m}^{n} \widehat{\psi_{m}}\left(N_{m} \xi\right)$ and supp $\widehat{\psi^{(m)}} \subset\left\{\xi: \frac{1}{\lambda_{1}} \leqslant|\xi| \leqslant \lambda_{1}\right\}$ which implies, in particular,

$$
D^{\alpha} \widehat{\psi^{(m)}}(0)=0 \quad \text { for any multi-index } \quad \alpha .
$$

Moreover, by (4.35) it follows that for any multi-index $\alpha$, there is a constant $c_{\alpha}$ such that

$$
\left|D^{\alpha} \widehat{\psi^{(m)}}(\xi)\right| \leqslant c_{\alpha} N_{m}^{n} \quad \text { for any } \quad m \geqslant 1 \text { and for any } \xi \in \mathbb{R}^{n} .
$$

Writing, as usual, $\psi_{t}^{(m)}(x)=t^{-n} \psi^{(m)}\left(t^{-1} x\right)$ we have

$$
\left(\psi_{m}\left(N_{j}^{-1} \cdot\right) * k\right)(u)=\left(\psi^{(m)}\left(N_{m} N_{j}^{-1} \cdot\right) * k\right)(u)=\left(N_{j} N_{m}^{-1}\right)^{n}\left(\psi_{N_{j} N_{m}^{-1}}^{(m)} * k\right)(u) .
$$


So (4.39) becomes

$$
I_{j m} \leqslant c\left(N_{j} N_{m}^{-1}\right)^{-r} N_{j}^{-n}\left(N_{j} N_{m}^{-1}\right)^{n} \cdot \sup _{u \in \mathbb{R}^{n}}\left(\left|\left(\psi_{N_{j} N_{m}^{-1}}^{(m)} * k\right)(u)\right|(1+|u|)^{L}\right) .
$$

Since $m>j$ we can use Lemma 4.3.1 with $t=N_{j} N_{m}^{-1}, \mu=\psi^{(m)} \in \diamond_{M}$ (where $M$ can be chosen arbitrary large due to (4.40)) and $\eta=k$ and obtain

$$
\begin{aligned}
& \sup _{u \in \mathbb{R}^{n}}\left(\left|\left(\psi_{N_{j} N_{m}^{-1}}^{(m)} * k\right)(u)\right|(1+|u|)^{L}\right) \leqslant c_{L}\left(N_{j} N_{m}^{-1}\right)^{M+1} . \\
& \max _{M+1 \leqslant|\beta| \leqslant \max (M+1, L+1)}\left\|D^{\beta} \widehat{\psi^{(m)}}\left|L_{\infty} \| \cdot \max _{|\alpha| \leqslant L+1} \int_{\mathbb{R}^{n}}(1+|\xi|)^{M+1}\right| D^{\alpha} \widehat{k}(\xi) \mid d \xi,\right.
\end{aligned}
$$

and using (4.41) we have, with a positive constant $c^{\prime}>0$,

$$
\sup _{u \in \mathbb{R}^{n}}\left(\left|\left(\psi_{N_{j} N_{m}^{-1}}^{(m)} * k\right)(u)\right|(1+|u|)^{L}\right) \leqslant c^{\prime}\left(N_{j} N_{m}^{-1}\right)^{M+1} N_{m}^{n} .
$$

Inserting the last inequality in (4.42) we finally obtain

$$
I_{j m} \leqslant c\left(N_{j} N_{m}^{-1}\right)^{-r} N_{j}^{-n}\left(N_{j} N_{m}^{-1}\right)^{n}\left(N_{j} N_{m}^{-1}\right)^{M+1} N_{m}^{n}=c\left(N_{j} N_{m}^{-1}\right)^{-r+M+1} .
$$

Recall that by (4.40) we may choose $M$ as large as we want. We choose $M$, an integer of the form

$$
M=-1+2 r+s \quad \text { with a real } s \text { satisfying } \quad s \log _{2} \lambda_{0}+\log _{2} d_{0}>0
$$

(note that such an $s$ exists due to the fact that $\lambda_{0}>1$ ), and (4.44) can be written

$$
I_{j m} \leqslant c\left(N_{j} N_{m}^{-1}\right)^{s+r} .
$$

Further, note that, for all $x, y \in \mathbb{R}^{n}$,

$$
\begin{aligned}
\left(\theta_{N_{m}^{-1}}^{*} f\right)_{r}(y) & \leqslant\left(\theta_{N_{m}^{-1}}^{*} f\right)_{r}(x)\left(1+N_{m}|x-y|\right)^{r} \\
& \leqslant\left(\theta_{N_{m}^{-1}}^{*} f\right)_{r}(x) \cdot \max \left(1,\left(N_{j}^{-1} N_{m}\right)^{r}\right) \cdot\left(1+N_{j}|x-y|\right)^{r} .
\end{aligned}
$$

Inserting the last inequality in (4.37), then dividing by $\left(1+N_{j}|x-y|\right)^{r}$ and using the estimates (4.38) and (4.46) for $I_{j m}$ we have

$$
\begin{aligned}
& \sup _{y \in \mathbb{R}^{n}} \frac{\left|\left(k_{N_{j}^{-1}} * \psi_{m} * \theta_{N_{m}^{-1}} * f\right)(y)\right|}{\left(1+N_{j}|x-y|\right)^{r}} \\
\leqslant & c\left(\theta_{N_{m}^{-1}}^{*} f\right)_{r}(x) \cdot \max \left(1,\left(N_{j}^{-1} N_{m}\right)^{r}\right) \cdot \begin{cases}\left(N_{j}^{-1} N_{m}\right)^{K+1} & \text { if } m \leqslant j \\
\left(N_{j} N_{m}^{-1}\right)^{s+r} & \text { if } m>j\end{cases} \\
= & c^{\prime}\left(\theta_{N_{m}^{-1}}^{*} f\right)_{r}(x) \cdot \begin{cases}\left(N_{j}^{-1} N_{m}\right)^{K+1} & \text { if } m \leqslant j \\
\left(N_{j} N_{m}^{-1}\right)^{s} & \text { if } m>j\end{cases}
\end{aligned}
$$


Note that in the above computations we did not use moment conditions for the function $\widehat{\psi_{1}}$. So, replacing $\psi_{1}$ and $\theta_{1}$ with $\psi_{0}$ and $\theta_{0}$ we get the similar estimate

$$
\sup _{y \in \mathbb{R}^{n}} \frac{\left|\left(k_{N_{j}^{-1}} * \psi_{0} * \theta_{0} * f\right)(y)\right|}{\left(1+N_{j}|x-y|\right)^{r}} \leqslant c\left(\theta_{0}^{*} f\right)_{r}(x)\left(N_{j}^{-1} N_{0}\right)^{K+1} .
$$

Using now (4.47) and (4.48) in (4.36), after multiplying with $\sigma_{j}$, we have

$$
\begin{aligned}
& \sigma_{j}\left(k_{N_{j}^{-1}}^{*} f\right)_{r}(x) \leqslant c\left(\theta_{0}^{*} f\right)_{r}(x) \sigma_{j} N_{j}^{-(K+1)} \\
+ & c^{\prime} \sum_{m=1}^{\infty}\left(\theta_{N_{m}^{-1}}^{*} f\right)_{r}(x) \cdot\left\{\begin{array}{ll}
\sigma_{j}\left(N_{j}^{-1} N_{m}\right)^{K+1} & \text { if } m \leqslant j \\
\sigma_{j}\left(N_{j} N_{m}^{-1}\right)^{s} & \text { if } m>j
\end{array},\right.
\end{aligned}
$$

with some positive constants $c, c^{\prime}$ independent of $j$ and $m$.

Let $m<j$. Then, after using (4.1) and (4.3) we have

$$
\begin{aligned}
\sigma_{j}\left(N_{j}^{-1} N_{m}\right)^{K+1} & \leqslant d_{1}^{j-m} \sigma_{m} \cdot \lambda_{0}-(j-m)(K+1) \\
& =\sigma_{m} \cdot 2^{-(j-m)\left[-\log _{2} d_{1}+(K+1) \log _{2} \lambda_{0}\right]} .
\end{aligned}
$$

Let now $m \geqslant j$. Again, using (4.1) and (4.3), we have

$$
\begin{aligned}
\sigma_{j}\left(N_{j} N_{m}^{-1}\right)^{s} & \leqslant d_{0}^{-(m-j)} \sigma_{m} \cdot \lambda_{0}^{-(m-j) s} \\
& =\sigma_{m} \cdot 2^{-(m-j)\left(\log _{2} d_{0}+s \log _{2} \lambda_{0}\right)} .
\end{aligned}
$$

Moreover,

$$
\sigma_{j} N_{j}^{-(K+1)} \leqslant d_{1}^{j} \sigma_{0} \cdot \lambda_{0}^{-j(K+1)} N_{0}^{-(K+1)}=\sigma_{0} N_{0}^{-(K+1)} 2^{-j\left[-\log _{2} d_{1}+(K+1) \log _{2} \lambda_{0}\right]} .
$$

Note that due to (4.45) and to our assumption on $K$, we have

$$
\varrho=\min \left\{-\log _{2} d_{1}+(K+1) \log _{2} \lambda_{0}, s \log _{2} \lambda_{0}+\log _{2} d_{0}\right\}>0 .
$$

Inserting the last two estimates in (4.49) we get, for all $f \in \ell^{\prime}$, all $x \in \mathbb{R}^{n}$ and all $j \in \mathbb{N}$,

$$
\sigma_{j}\left(k_{N_{j}^{-1}}^{*} f\right)_{r}(x) \leqslant c \sigma_{0}\left(\theta_{0}^{*} f\right)_{r}(x) 2^{-j \varrho}+c^{\prime} \sum_{m=1}^{\infty} \sigma_{m}\left(\theta_{N_{m}^{-1}}^{*} f\right)_{r}(x) \cdot 2^{-|j-m| \varrho} .
$$

Again for $j=1$ we did not use moment conditions to obtain this estimate so we can replace $k_{N_{1}^{-1}}$ with $k_{0}$ and get

$$
\left(k_{0}^{*} f\right)_{r}(x) \leqslant c\left(\theta_{0}^{*} f\right)_{r}(x)+c^{\prime} \sum_{m=1}^{\infty} \sigma_{m}\left(\theta_{N_{m}^{-1}}^{*} f\right)_{r}(x) \cdot 2^{-m \varrho} .
$$

The estimate (4.33) follows now as a simple consequence of the elementary Lemma 4.3.2.

Consequently we have finished the proof of the inequality (4.33). 
Step 2. Take again $\left(\varphi_{j}^{N}\right)_{j \in \mathbb{N}_{0}}$ a fixed partition of unity associated to $\left(N_{j}\right)_{j \in \mathbb{N}_{0}}$, that means $\left(\varphi_{j}^{N}\right)_{j \in \mathbb{N}_{0}}$ has the properties (2.7)-(2.10) with $c_{\varphi}=1$.

For a fixed $j \in \mathbb{N}_{0}$ let

$$
\Phi_{j}(\xi)=\sum_{m=0}^{j} \varphi_{m}^{N}(\xi)
$$

Using the properties of the system $\left(\varphi_{j}^{N}\right)_{j \in \mathbb{N}_{0}}$ we have $\Phi_{j}(\xi)=1$ if $|\xi|<N_{j-1}$, $\Phi_{j}(\xi)=0$ if $|\xi|>N_{j+1}$ and for any multi-index $\alpha$ there exists a constant $c_{\alpha}$ (independent of $j$ ) such that

$$
\left|D^{\alpha} \Phi_{j}(\xi)\right| \leqslant c_{\alpha}\langle\xi\rangle^{-|\alpha|} .
$$

Let us consider now the function $\Psi_{j}$ defined by

$$
\widehat{\Psi_{j}}(\xi)=\frac{\Phi_{j}(\xi)}{\widehat{k_{0}}\left(N_{j}^{-1} \xi\right)}, j \in \mathbb{N}_{0}
$$

Note that, for $|\xi| \leqslant N_{j+1} \leqslant \lambda_{1} N_{j}$, it follows that $N_{j}^{-1}|\xi| \leqslant \lambda_{1} \leqslant N_{1}$, and due to the assumption (4.22) on $k_{0}$, this shows that $\Psi_{j}$ is well defined.

Clearly one has $\operatorname{supp} \widehat{\Psi_{j}} \subset\left\{\xi \in \mathbb{R}^{n}:|\xi| \leqslant N_{j+1}\right\}$ since $\Phi_{j}(\xi)=0$ if $|\xi|>N_{j+1}$.

Moreover, applying Leibniz's rule for differentiation of a product we get that, for any multi-index $\gamma$, there exists a constant $c_{\gamma}>0$ independent of $j$ such that

$$
\left|D^{\gamma}\left[\widehat{\Psi_{j}}\left(N_{j} \xi\right)\right]\right| \leqslant \sum_{\delta \leqslant \gamma} c_{\gamma \delta} D^{\delta}\left[\Phi_{j}\left(N_{j} \xi\right)\right] D^{\gamma-\delta}\left[\frac{1}{\widehat{k_{0}}(\xi)}\right] \leqslant c_{\gamma} .
$$

From (4.50) we get that, for any $f \in \varsigma^{\prime}$, we have

$$
\Psi_{j} * N_{j}^{n} k_{0}\left(N_{j} \cdot\right) * f=\check{\Phi}_{j} * f .
$$

Later on we will use the notation $\left(k_{0}\right)_{N_{j}^{-1}}(x)=N_{j}^{n} k_{0}\left(N_{j} x\right)$.

On the other hand, for a fixed $j$, we define, for any $m \geqslant j+1$, the functions $\psi_{m}$ by

$$
\widehat{\psi_{m}}(\xi)=\frac{\varphi_{m}^{N}(\xi)}{\widehat{k}\left(N_{m}^{-1} \xi\right)}
$$

in analogy to the first step, see (4.34), now with $k$ instead of $\theta$. Consequently we have for any $f \in \wp^{\prime}$ (and for a fixed $j$ ),

$$
f=\Psi_{j} *\left(k_{0}\right)_{N_{j}^{-1}} * f+\sum_{m=j+1}^{\infty} \psi_{m} * k_{N_{m}^{-1}} * f
$$

and this implies

$$
k_{N_{j}^{-1}} * f=\left(\Psi_{j} *\left(k_{0}\right)_{N_{j}^{-1}}\right) *\left(k_{N_{j}^{-1}} * f\right)+\sum_{m=j+1}^{\infty}\left(k_{N_{j}^{-1}} * \psi_{m}\right) *\left(k_{N_{m}^{-1}} * f\right) .
$$


Now $\left(\Psi_{j} *\left(k_{0}\right)_{N_{j}^{-1}}\right)(z)=\left(k_{0} * \Psi_{j}\left(N_{j}^{-1} \cdot\right)\right)\left(N_{j} z\right)$ and we may apply Lemma 4.3.1 with $t=1, M=-1, \mu=k_{0}$ and $\eta=\Psi_{j}\left(N_{j}^{-1} \cdot\right)$. So, for any $L>0$,

$$
\begin{aligned}
& \sup _{z \in \mathbb{R}^{n}}\left(\left|\left(\Psi_{j} *\left(k_{0}\right)_{N_{j}^{-1}}\right)(z)\right| \cdot\left(1+N_{j}|z|\right)^{L}\right) \\
= & \sup _{z \in \mathbb{R}^{n}}\left(\left|\left(k_{0} * \Psi_{j}\left(N_{j}^{-1} \cdot\right)\right)\left(N_{j} z\right)\right| \cdot\left(1+N_{j}|z|\right)^{L}\right) \\
\leqslant & c_{L} \cdot 1^{0} \cdot \max _{0 \leqslant|\beta| \leqslant \max (0, L+1)}|| D^{\beta} \widehat{k_{0}}\left|L_{\infty} \| \cdot \max _{|\gamma| \leqslant L+1} \int_{\mathbb{R}^{n}}\right| D^{\gamma}\left[\Psi_{j}\left(N_{j}^{-1} \cdot\right)\right] \widehat{(\xi)} \mid d \xi \\
\leqslant & c^{\prime} \max _{|\gamma| \leqslant L+1} N_{j}^{n} \int_{|\xi| \leqslant \lambda_{1}}\left|D^{\gamma}\left[\widehat{\Psi_{j}}\left(N_{j} \xi\right)\right]\right| d \xi \\
\leqslant & c^{\prime \prime} N_{j}^{n},
\end{aligned}
$$

where $c^{\prime \prime}>0$ is independent of $j$ and in the last two inequalities we have used the properties of the function $\widehat{\Psi}_{j}$, in particular the localisation of its support and (4.51). Consequently, for any $L>0$ there is a positive constant $C_{L}>0$ such that

$$
\left|\left(\Psi_{j} *\left(k_{0}\right)_{N_{j}^{-1}}\right)(z)\right| \leqslant C_{L} \frac{N_{j}^{n}}{\left(1+N_{j}|z|\right)^{L}}, \quad z \in \mathbb{R}^{n} .
$$

Writing, for any $m \geqslant j+1$,

$$
\left(k_{N_{j}^{-1}} * \psi_{m}\right)(u)=\left(N_{j} N_{m}^{-1}\right)^{n}\left(\psi_{N_{j} N_{m}^{-1}}^{(m)} * k\right)\left(N_{j} u\right),
$$

where $\psi^{(m)}=\psi_{m}\left(N_{m}^{-1} u\right)$, we get as in Step 1, compare (4.39) and (4.43), that for any $M$ and $L$ there is a constant $c>$ independent of $j$ and $m$

$$
\left|\left(k_{N_{j}^{-1}} * \psi_{m}\right)(z)\right| \leqslant c \frac{N_{j}^{n}\left(N_{j} N_{m}^{-1}\right)^{M}}{\left(1+N_{j}|z|\right)^{L}}, \quad z \in \mathbb{R}^{n} .
$$

Inserting the estimates (4.53) and (4.54) with $r=L$ in (4.52) we get, for all $f \in \varsigma^{\prime}$, $y \in \mathbb{R}^{n}$ and $j \in \mathbb{N}$,

$$
\begin{aligned}
\left|\left(k_{N_{j}^{-1}} * f\right)(y)\right| \leqslant & c \int_{\mathbb{R}^{n}} \frac{N_{j}^{n}}{\left(1+N_{j}|y-z|\right)^{r}}\left|\left(k_{N_{j}^{-1}} * f\right)(z)\right| d z \\
& +c^{\prime} \sum_{m=j+1}^{\infty} \int_{\mathbb{R}^{n}} \frac{N_{j}^{n}\left(N_{j} N_{m}^{-1}\right)^{M}}{\left(1+N_{j}|y-z|\right)^{r}}\left|\left(k_{N_{m}^{-1}} * f\right)(z)\right| d z \\
\leqslant & C \sum_{m=j}^{\infty} N_{j}^{n}\left(N_{j} N_{m}^{-1}\right)^{M} \int_{\mathbb{R}^{n}} \frac{\left|\left(k_{N_{m}^{-1}} * f\right)(z)\right|}{\left(1+N_{j}|y-z|\right)^{r}} d z .
\end{aligned}
$$

Fix now any $\varrho \in(0,1]$. We divide both sides of (4.55) by $\left(1+N_{j}|x-y|\right)^{r}$, then, on the left-hand side we take the supremum over $y \in \mathbb{R}^{n}$ and on the right hand-side 
we use the inequalities

$$
\begin{gathered}
\left(1+N_{j}|x-y|\right)\left(1+N_{j}|y-z|\right) \geqslant 1+N_{j}|x-z|, \\
\left|\left(k_{N_{m}^{-1}} * f\right)(z)\right| \leqslant\left|\left(k_{N_{m}^{-1}} * f\right)(z)\right|^{\varrho} \cdot\left[\left(k_{N_{m}^{-1}}^{*} f\right)_{r}(x)\right]^{1-\varrho} \cdot\left(1+N_{m}|x-z|\right)^{r(1-\varrho)},
\end{gathered}
$$

and

$$
\frac{\left(1+N_{m}|x-z|\right)^{r(1-\varrho)}}{\left(1+N_{j}|x-z|\right)^{r}} \leqslant \frac{\left(N_{j}^{-1} N_{m}\right)^{r}}{\left(1+N_{m}|x-z|\right)^{r \varrho}}
$$

and get, for all $f \in \varsigma^{\prime}$, all $x \in \mathbb{R}^{n}$ and all $j \in \mathbb{N}$,

$$
\left(k_{N_{j}^{-1}}^{*} f\right)_{r}(x) \leqslant c \sum_{m=j}^{\infty}\left(N_{j} N_{m}^{-1}\right)^{A} \int_{\mathbb{R}^{n}} \frac{N_{m}^{n} \cdot\left|\left(k_{N_{m}^{-1}} * f\right)(z)\right|^{\varrho}}{\left(1+N_{m}|x-z|\right)^{r \varrho}} d z \cdot\left[\left(k_{N_{m}^{-1}}^{*} f\right)_{r}(x)\right]^{1-\varrho},
$$

where $A=M-r+n$ can be still taken arbitrary large.

Quite analogously one proves, for all $f \in \wp^{\prime}$, the estimate

$$
\begin{aligned}
\left(k_{0}^{*} f\right)_{r}(x) & \leqslant c\left(\int_{\mathbb{R}^{n}} \frac{\left|\left(k_{0} * f\right)(z)\right|^{\varrho}}{(1+|x-z|)^{r \varrho}} d z \cdot\left[\left(k_{0}^{*} f\right)_{r}(x)\right]^{1-\varrho}\right. \\
& \left.+\sum_{m=1}^{\infty} N_{m}^{-A} \int_{\mathbb{R}^{n}} \frac{N_{m}^{n} \cdot\left|\left(k_{N_{m}^{-1}} * f\right)(z)\right|^{\varrho}}{\left(1+N_{m}|x-z|\right)^{r \varrho}} d z \cdot\left[\left(k_{0}^{*} f\right)_{r}(x)\right]^{1-\varrho}\right) .
\end{aligned}
$$

At this moment we need Lemma 4.3.3. We fix $x \in \mathbb{R}^{n}$ and apply Lemma 4.3.3 with

$$
\begin{gathered}
a_{m}=\left(k_{N_{m}^{-1}}^{*} f\right)_{r}(x), \quad m \in \mathbb{N}, \quad a_{0}=\left(k_{0}^{*} f\right)_{r}(x), \\
b_{m}=\int_{\mathbb{R}^{n}} \frac{N_{m}^{n}\left|\left(k_{N_{m}^{-1}} * f\right)(z)\right|^{\varrho}}{\left(1+N_{m}|x-z|\right)^{r \varrho}} d z, \quad b_{0}=\int_{\mathbb{R}^{n}} \frac{\left|\left(k_{0} * f\right)(z)\right|}{(1+|x-z|)^{r \varrho}} d z .
\end{gathered}
$$

The assumption (4.18) is satisfied with $A_{0}$ equal to the order of the distribution $f \in 8^{\prime}$. The estimates (4.57) and (4.58) take the form (4.19). Consequently (4.20) is true and this means that, for every $A>0$, there is a constant $c_{A}>0$ such that

$$
\left(k_{N_{j}^{-1}}^{*} f\right)_{r}(x)^{\varrho} \leqslant c_{A} \sum_{m=j}^{\infty}\left(N_{j} N_{m}^{-1}\right)^{A \varrho} \cdot \int_{\mathbb{R}^{n}} \frac{N_{m}^{n}\left|\left(k_{N_{m}^{-1}} * f\right)(z)\right|^{\varrho}}{\left(1+N_{m}|x-z|\right)^{r \varrho}} d z
$$

together with the corresponding estimate for $\left(k_{0}^{*} f\right)_{r}(x)$. Note that $c_{A}$ in (4.59) is independent of $f \in \mathcal{S}^{\prime}, x \in \mathbb{R}^{n}, j \in \mathbb{N}$ and $\varrho \in(0,1]$ because of Lemma 4.3.3.

Further note that (4.59) is also true for $\varrho>1$ with a simpler proof. It suffices to take $r+n$ instead of $r$, apply Hölder's inequality in $m$ and in $z$, and finally the inequality (4.56). We omit the details.

It is possible to choose $\varrho$ so that

$$
\frac{n}{r}<\varrho<\min (p, q) \quad \text { (respectively } \frac{n}{r}<\varrho<p \text { for Besov spaces). }
$$

We make such a choice and fix $\varrho$ for the rest of the proof. 
Now the function $z \mapsto \frac{1}{(1+|z|)^{r} \varrho}$ is in $L_{1}$ and we may use the majorant property for the Hardy-Littlewood maximal operator $\mathcal{M}$, see E.M. Stein and G. Weiss [StWe71, Chap. 2,(3.9)],

$$
\left(|g|^{\varrho} * \frac{N_{m}^{n}}{\left(1+N_{m}|\cdot|\right)^{r \varrho}}\right)(x) \leqslant \mathcal{M}\left(|g|^{\varrho}\right)(x) \cdot\left\|\frac{N_{m}^{n}}{\left(1+N_{m}|\cdot|\right)^{r \varrho}} \mid L_{1}\right\| .
$$

It follows from (4.59) that

$$
\left(k_{N_{j}^{-1}}^{*} f\right)_{r}(x)^{\varrho} \leqslant c \sum_{m=j}^{\infty}\left(N_{j} N_{m}^{-1}\right)^{A \varrho} \cdot \mathcal{M}\left(\left|k_{N_{m}^{-1}} * f\right|^{\varrho}\right)(x),
$$

together with the corresponding estimate for $\left(k_{0}^{*} f\right)_{r}(x)$.

Again we use that, for $m \geqslant j$,

$$
N_{j} N_{m}^{-1} \leqslant \lambda_{0}^{-(m-j)}=2^{-(m-j) \log _{2} \lambda_{0}}
$$

and

$$
\sigma_{j} \leqslant d_{0}^{-(m-j)} \sigma_{m}=2^{-(m-j) \log _{2} d_{0}},
$$

and so (4.60) becomes (with some positive constant $c$ )

$$
\sigma_{j}^{\varrho}\left(k_{N_{j}^{-1}}^{*} f\right)_{r}(x)^{\varrho} \leqslant c \sum_{m=j}^{\infty} 2^{-(m-j)\left(A \varrho \log _{2} \lambda_{0}+\varrho \log _{2} d_{0}\right)} \sigma_{m}^{\varrho} \cdot \mathcal{M}\left(\left|k_{N_{m}^{-1}} * f\right|^{\varrho}\right)(x) .
$$

We can choose $A>0$ large enough such that

$$
\varepsilon=A \varrho \log _{2} \lambda_{0}+\varrho \log _{2} d_{0}>0
$$

Now we apply Lemma 4.3.2 with

$$
g_{j}(x)=\sigma_{j}^{\varrho} \mathcal{M}\left(\left|k_{N_{j}^{-1}} * f\right|^{\varrho}\right)(x), \quad j \in \mathbb{N}, \quad g_{0}=\mathcal{M}\left(\left|k_{0} * f\right|^{\varrho}\right)
$$

in $L_{p / \varrho}\left(l_{q / \varrho}\right)$ and get, from (4.61),

$$
\begin{aligned}
& \left\|\left(k_{0}^{*}\right)_{r}\left|L_{p}\|+\|\left(\sigma_{j}\left(k_{N_{j}^{-1}}^{*} f\right)_{r}\right)_{j \in \mathbb{N}}\right| L_{p}\left(l_{q}\right)\right\| \\
\leqslant & c\left(\left\|\mathcal{M}_{\varrho}\left(k_{0} * f\right)\left|L_{p}\|+\|\left(\sigma_{j} \mathcal{M}_{\varrho}\left(k_{N_{j}^{-1}} * f\right)\right)_{j \in \mathbb{N}}\right| L_{p}\left(l_{q}\right)\right\|\right),
\end{aligned}
$$

where we used the notation $\mathcal{M}_{\varrho}(g)=\mathcal{M}\left(|g|^{\varrho}\right)^{1 / \varrho}$.

By the maximal inequality of C. Fefferman and E.M. Stein, see [FeSt71], we know that $\mathcal{M}_{\varrho}$ is a bounded operator

$$
\mathcal{M}_{\varrho}: L_{p}\left(l_{q}\right) \rightarrow L_{p}\left(l_{q}\right), \quad \varrho<p<\infty, \quad \varrho<q \leqslant \infty
$$

(respectively $\left.\mathcal{M}_{\varrho}: l_{q}\left(L_{p}\right) \rightarrow l_{q}\left(L_{p}\right), \quad \varrho<p \leqslant \infty, \quad 0<q \leqslant \infty\right)$. Our choice of $\varrho$ enables us to apply (4.62) and we obtain (with some positive constant $C$ ) 


$$
\begin{aligned}
& \left\|\left(k_{0}^{*} f\right)_{r}\left|L_{p}\|+\|\left(\sigma_{j}\left(k_{N_{j}^{-1}}^{*} f\right)_{r}\right)_{j \in \mathbb{N}}\right| L_{p}\left(l_{q}\right)\right\| \\
\leqslant & C\left(\left\|k_{0} * f\left|L_{p}\|+\|\left(\sigma_{j}\left(k_{N_{j}^{-1}} * f\right)\right)_{j \in \mathbb{N}}\right| L_{p}\left(l_{q}\right)\right\|\right) \text { for any } f \in \mathcal{s}^{\prime} .
\end{aligned}
$$

A corresponding inequality is obtained for the spaces $l_{q}\left(L_{p}\right)$.

Step 3. Let $\mu_{0}$ and $\mu \in \S$ be two positive functions on $\mathbb{R}^{n}$ satisfying (4.5) and (4.6). Let

$$
\widehat{\theta_{0}}=\mu_{0} \quad \text { and } \quad \widehat{\theta}=\mu .
$$

We have successively

$$
\begin{aligned}
& \left\|\left(k_{0}^{*} f\right)_{r}\left|L_{p}\|+\|\left(\sigma_{j}\left(k_{N_{j}^{-1}}^{*} f\right)_{r}\right)_{j \in \mathbb{N}}\right| L_{p}\left(l_{q}\right)\right\| \\
\leqslant & c\left(\left\|\left(\theta_{0}^{*} f\right)_{r}\left|L_{p}\|+\|\left(\sigma_{j}\left(\theta_{N_{j}^{-1}}^{*} f\right)_{r}\right)_{j \in \mathbb{N}}\right| L_{p}\left(l_{q}\right)\right\|\right) \\
\leqslant & c_{1}\left(\left\|\theta_{0} * f\left|L_{p}\|+\|\left(\sigma_{j}\left(\theta_{N_{j}^{-1}} * f\right)\right)_{j \in \mathbb{N}}\right| L_{p}\left(l_{q}\right)\right\|\right) \\
\leqslant & c_{2}\left\|f \mid F_{p, q}^{\sigma, N}\right\|,
\end{aligned}
$$

where the first inequality is (4.33), see Step 1; the second inequality is (4.63) (with $\theta_{0}$ and $\theta$ instead of $k_{0}$ and $k$ ), see Step 2; and finally the last inequality is nothing else than (4.9), see Theorem 4.2.2, since $\theta_{0} * f=\left(\mu_{0} \widehat{f}\right)^{\vee}$ and $\theta_{N_{j}^{-1}} * f=\left(\mu_{j} \widehat{f}\right)^{\vee}$. Consequently we have proved (4.28).

Moreover,

$$
\begin{aligned}
\left\|f \mid F_{p, q}^{\sigma, N}\right\| & \leqslant c\left(\left\|\left(\theta_{0}^{*} f\right)_{r}\left|L_{p}\|+\|\left(\sigma_{j}\left(\theta_{N_{j}^{-1}}^{*} f\right)_{r}\right)_{j \in \mathbb{N}}\right| L_{p}\left(l_{q}\right)\right\|\right) \\
& \leqslant c_{1}\left(\left\|\left(k_{0}^{*} f\right)_{r}\left|L_{p}\|+\|\left(\sigma_{j}\left(k_{N_{j}^{-1}}^{*} f\right)_{r}\right)_{j \in \mathbb{N}}\right| L_{p}\left(l_{q}\right)\right\|\right) \\
& \leqslant c_{2}\left(\left\|\left(k_{0} * f\right)\left|L_{p}\|+\|\left(\sigma_{j}\left(k_{N_{j}^{-1}} * f\right)\right)_{j \in \mathbb{N}}\right| L_{p}\left(l_{q}\right)\right\|\right),
\end{aligned}
$$

where the first inequality is an obvious consequence of (4.9), see Theorem 4.2.2, the second inequality is (4.33), see Step 1 , with the roles of $k_{0}$ and $k$, respectively, $\theta_{0}$ and $\theta$ interchanged, and finally the last inequality is (4.63), see Step 2.

Consequently we have proved (4.29), too.

4.3.4. Comments. We would like to point out that we used at several places the fact that the sequence $N$ is of bounded growth.

The above argument follows essentially H.-Q. Bui, M. Paluszyński and M. Taibleson, see [BPT96] and [BPT97], and the simplified version of their papers given by V. Rychkov in [Ry99]. However, due to the general structure of the sequences $\left(N_{j}\right)_{j \in \mathbb{N}_{0}}$ satisfying (4.1) we had to adapt some steps of their proof.

First, the key Lemma 4.3.1 is related to Lemma 2.1 in [BPT96] and to Lemma 1 in [Ry99] but we needed to indicate the dependence of $\mu$ and $\eta$ of the factor that multiplies $t^{M+1}$. 
Secondly, the argument of Step 1 and the idea of proving (4.33) essentially go back to J. Peetre, see [Pe75]. Compared with the classical situation $\left(N_{j}=2^{j}\right.$ for any $j \in \mathbb{N}_{0}$ ) and with the proof in [Ry99], to estimate the integral $I_{j m}$ in (4.37) for $m>j$ we had to take into account that the functions $\psi_{m}$ are not generated from a single function $\psi$. This caused complications which were solved applying Lemma 4.3.1 in the form which was stated.

As a third observation we point out, see Step 2, that in order to prove (4.63) we had to introduce the function $\Phi_{j}$ to obtain the equality (4.52). This allowed us to avoid the dilation argument from [Ry99] which could not work in the case of general sequences $\left(N_{j}\right)_{j \in \mathbb{N}_{0}}$.

Finally, note that the above technique to prove the estimate (4.59) (if $N_{j}=2^{j}$ for any $j \in \mathbb{N}_{0}$ ) is due to J.-O. Strömberg and A. Torchinsky, see [StTo89, Chap. 5, Theorem 2(a)].

Remark 4.3.6. Theorem 4.3.4 paves the way to the proof of the atomic decomposition theorem, see next section, but it is of independent interest since it covers the classical results of H.-Q. Bui, M. Paluszyński and M. Taibleson, see [BPT96] and [BPT97], the theorem on local means from [Tr92, Theorem 2.4.6], and the theorem on local means from [Mo99] and [Mo01]. We will return to this aspect later on.

\subsection{N-atoms and the atomic decomposition theorem}

4.4.1. Preliminaries: $N$-atoms and sequence spaces. Recall that $N=\left(N_{j}\right)_{j \in \mathbb{N}_{0}}$ is an admissible sequence with bounded growth which satisfies (4.1) with $\lambda_{0}>1$.

Let $\mathbb{Z}^{n}$ be the lattice of all points in $\mathbb{R}^{n}$ with integer-valued components.

If $v \in \mathbb{N}_{0}$ and $m=\left(m_{1}, \ldots, m_{n}\right) \in \mathbb{Z}^{n}$ we denote $Q_{v m}$ the cube in $\mathbb{R}^{n}$ centred at $N_{v}^{-1} m=\left(N_{v}^{-1} m_{1}, \ldots, N_{v}^{-1} m_{n}\right)$ which has sides parallel to the axes and side length $N_{v}^{-1}$.

If $Q_{\nu m}$ is such a cube in $\mathbb{R}^{n}$ and $c>0$ then $c Q_{\nu m}$ is the cube in $\mathbb{R}^{n}$ concentric with $Q_{\nu m}$ and with side length $c N_{v}^{-1}$.

We are now prepared to introduce the $N$-atoms (associated to the sequence $N$ ).

Definition 4.4.1. (i) Let $M \in \mathbb{R}, c^{*}>1$. A function $\rho: \mathbb{R}^{n} \rightarrow \mathbb{C}$, for which there exist all derivatives $D^{\alpha} \rho$ if $|\alpha| \leqslant M$ (continuous if $M \leqslant 0$ ), is called an $1_{M}$-N-atom if

$$
\begin{gathered}
\text { supp } \rho \subset c^{*} Q_{0 m} \quad \text { for some } m \in \mathbb{Z}^{n}, \\
\left|D^{\alpha} \rho(x)\right| \leqslant 1 \quad \text { if }|\alpha| \leqslant M .
\end{gathered}
$$

(ii) Let $\sigma=\left(\sigma_{j}\right)_{j \in \mathbb{N}_{0}}$ be an admissible sequence, let $0<p \leqslant \infty, M, L \in \mathbb{R}$, $c^{*}>1$. A function $\rho: \mathbb{R}^{n} \rightarrow \mathbb{C}$, for which there exist all derivatives $D^{\alpha} \rho$ if $|\alpha| \leqslant M$ (continuous if $M \leqslant 0)$, is called an $(\sigma, p)_{M, L}-N$-atom if

$$
\text { supp } \rho \subset c^{*} Q_{\nu m} \text { for some } \quad v \in \mathbb{N}, m \in \mathbb{Z}^{n},
$$




$$
\begin{gathered}
\left|D^{\alpha} \rho(x)\right| \leqslant \sigma_{\nu}^{-1} N_{\nu}^{\frac{n}{p}+|\alpha|} \quad \text { if } \quad|\alpha| \leqslant M, \\
\int_{\mathbb{R}^{n}} x^{\gamma} \rho(x) d x=0 \quad \text { if } \quad|\gamma| \leqslant L .
\end{gathered}
$$

If the atom $\rho$ is located at $Q_{v m}$ (that means supp $\rho \subset c^{*} Q_{v m}$ with $v \in \mathbb{N}_{0}$, $m \in \mathbb{Z}^{n}, c^{*}>1$ ) then we will denote it by $\rho_{v m}$.

This concept generalises the smooth (isotropic) atoms from the works of M. Frazier and B. Jawerth, [FrJa85] and [FrJa90], which correspond to $N_{v}=2^{v}$ and $\sigma_{v}=2^{v s}$ with real $s$.

We give some technical explanations.

The value of the number $c^{*}>1$ in (4.64) and (4.66) is unimportant. It simply makes clear that at the level $v$ some controlled overlapping of the supports of $\rho_{v m}$ must be allowed.

The moment conditions (4.68) can be reformulated as $D^{\gamma} \widehat{\rho}(0)=0$ if $|\gamma| \leqslant L$, which shows that a sufficiently strong decay of $\widehat{\rho}$ at the origin is required. If $L<0$ then (4.68) simply means that there are no moment conditions.

The reason for the normalising factor in (4.65) and (4.67) is that there exists a constant $c>0$ such that, for all these atoms, we have $\left\|\rho \mid B_{p, q}^{\sigma, N}\right\| \leqslant c$ and $\left\|\rho \mid F_{p, q}^{\sigma, N}\right\| \leqslant c$. Hence, as in the classical case, atoms are normalised building blocks satisfying some moment conditions.

Before we state the atomic decomposition theorem we have to introduce the sequence spaces $b_{p, q}$ and $f_{p, q}^{N}$.

If $v \in \mathbb{N}_{0}, m \in \mathbb{Z}^{n}$ and $Q_{v m}$ is a cube as above let $\chi_{v m}$ be the characteristic function of $Q_{v m}$; if $0<p \leqslant \infty$ let

$$
\chi_{v m}^{(p)}=N_{v}^{n / p} \chi_{v m}
$$

(obvious modification if $p=\infty$ ) be the $L_{p}$-normalised characteristic function of $Q_{v m}$.

Definition 4.4.2. Let $0<p \leqslant \infty, 0<q \leqslant \infty$. Then:

(i) $b_{p, q}$ is the collection of all sequences $\lambda=\left\{\lambda_{v m} \in \mathbb{C}: v \in \mathbb{N}_{0}, m \in \mathbb{Z}^{n}\right\}$ such that

$$
\left\|\lambda \mid b_{p, q}\right\|=\left(\sum_{\nu=0}^{\infty}\left(\sum_{m \in \mathbb{Z}^{n}}\left|\lambda_{v m}\right|^{p}\right)^{q / p}\right)^{1 / q}
$$

(with the usual modification if $p=\infty$ and/or $q=\infty$ ) is finite;

(ii) $f_{p, q}^{N}$ is the collection of all sequences $\lambda=\left\{\lambda_{v m} \in \mathbb{C}: v \in \mathbb{N}_{0}, m \in \mathbb{Z}^{n}\right\}$ such that

$$
\left\|\lambda\left|f_{p, q}^{N}\|=\|\left(\sum_{\nu=0}^{\infty} \sum_{m \in \mathbb{Z}^{n}}\left|\lambda_{v m} \chi_{v m}^{(p)}(\cdot)\right|^{q}\right)^{1 / q}\right| L_{p}\right\|
$$

(with the usual modification if $p=\infty$ and/or $q=\infty$ ) is finite.

One can easily see that $b_{p, q}$ and $f_{p, q}^{N}$ are quasi-Banach spaces and using $\left\|\chi_{v m}^{(p)} \mid L_{p}\right\|=1$ it is clear that, comparing $\left\|\lambda \mid b_{p, q}\right\|$ and $\left\|\lambda \mid f_{p, q}^{N}\right\|$, the roles of the quasi-norms in $L_{p}$ and $l_{q}$ are interchanged. 
4.4.2. The atomic decomposition theorem. We are able now to state the main result of this section.

Theorem 4.4.3. Let $N=\left(N_{j}\right)_{j \in \mathbb{N}_{0}}$ be an admissible sequence with $\lambda_{0}>1$ in (4.1) and let $\sigma=\left(\sigma_{j}\right)_{j \in \mathbb{N}_{0}}$ be an admissible sequence.

Let $0<p<\infty$, respectively $0<p \leqslant \infty, 0<q \leqslant \infty$, and let $M, L \in \mathbb{R}$ such that

$$
M>\frac{\log _{2} d_{1}}{\log _{2} \lambda_{0}}
$$

and

$$
L>-1+n\left(\frac{\log _{2} \lambda_{1}}{\log _{2} \lambda_{0}} \frac{1}{\min (1, p, q)}-1\right)-\frac{\log _{2} d_{0}}{\log _{2} \lambda_{0}},
$$

respectively

$$
L>-1+n\left(\frac{\log _{2} \lambda_{1}}{\log _{2} \lambda_{0}} \frac{1}{\min (1, p)}-1\right)-\frac{\log _{2} d_{0}}{\log _{2} \lambda_{0}} .
$$

Then $g \in \delta^{\prime}$ belongs to $F_{p, q}^{\sigma, N}$, respectively to $B_{p, q}^{\sigma, N}$, if and only if, it can be represented as

$$
g=\sum_{\nu=0}^{\infty} \sum_{m \in \mathbb{Z}^{n}} \lambda_{v m} \rho_{v m},
$$

convergence being in $\mathrm{\delta}^{\prime}$, where $\rho_{v m}$ are $1_{M}$-N-atoms $(v=0)$ or $(\sigma, p)_{M, L}-N$-atoms $(\nu \in \mathbb{N})$ and $\lambda \in f_{p, q}^{N}$, respectively $\lambda \in b_{p, q}$, where $\lambda=\left\{\lambda_{v m}: v \in \mathbb{N}_{0}, m \in \mathbb{Z}^{n}\right\}$. Furthermore, inf $\left\|\lambda \mid f_{p, q}^{N}\right\|$, respectively inf $\left\|\lambda \mid b_{p, q}\right\|$, where the infimum is taken over all admissible representations (4.72), is an equivalent quasi-norm in $F_{p, q}^{\sigma, N}$, respectively $B_{p, q}^{\sigma, N}$.

The convergence in $\delta^{\prime}$ can be obtained as a by-product of the proof using the same method as in [Tr97, Theorem 13.7], compare also the discussion in [Tr01], so we will not stress this point. We refer to the above theorem as to the atomic decomposition theorem in function spaces of generalised smoothness.

Before giving the proof let us make here some remarks. The first part of the proof, that one in which the atoms are constructed and where it is shown that the decomposition (4.72) holds, is essentially based on a version of a resolution of unity of Calderon type, cf. [FJW91, Lemma 5.12].

To prove the second part we will use the theorem on local means, see Theorem 4.3.4, the technique of maximal functions and an inequality of Fefferman-Stein type.

Remark 4.4.4. For spaces $B_{p, q}^{\omega}$ of positive smoothness, defined in the spirit of M.L. Goldman, see Section 3.3, an atomic decomposition in the sense of M. Frazier and B. Jawerth was described by Yu.V. Netrusov in [Ne89]. There are no moment conditions in his characterisation - in contrast to the case $0<p \leqslant 1$ in the above theorem. The reason is that Yu.V. Netrusov defined the spaces $B_{p, q}^{\omega}$ in a slightly different way which insures a priori the embedding $L_{p} \hookrightarrow B_{p, q}^{\omega}$ for all admissible parameters $0<p \leqslant \infty$. 
4.4.3. An auxiliary result: a partition of unity of Calderon type. We will need the following:

Lemma 4.4.5. Let $N=\left(N_{j}\right)_{j \in \mathbb{N}_{0}}$ be an admissible sequence with $\lambda_{0}>1$ in (4.1) and let $\left(\Omega_{j}^{N}\right)_{j \in \mathbb{N}_{0}}$ be the associated covering of $\mathbb{R}^{n}$ with $J=\kappa_{0}=1$, see (2.5) and (2.6).

Let also $\left(\varphi_{j}^{N}\right)_{j \in \mathbb{N}_{0}} \in \Phi^{N}$ be fixed with $c_{\varphi}=1$ and $L \geqslant 0$ be also fixed.

Then there exist functions $\theta_{0}, \theta \in \&$ with:

$$
\begin{gathered}
\operatorname{supp} \theta_{0} \text {, supp } \theta \subset\left\{x \in \mathbb{R}^{n}:|x| \leqslant 1\right\}, \\
\left|\widehat{\theta_{0}}(\xi)\right| \geqslant c_{0}>0 \quad \text { if }|\xi| \leqslant N_{1}, \\
|\widehat{\theta}(\xi)| \geqslant c>0 \quad \text { if } \quad \frac{1}{\lambda_{1}} \leqslant|\xi| \leqslant \lambda_{1}, \\
\int_{\mathbb{R}^{n}} x^{\gamma} \theta(x) d x=0 \text { if }|\gamma| \leqslant L,
\end{gathered}
$$

and

$$
\widehat{\theta_{0}}(\xi) \widehat{\psi_{0}}(\xi)+\sum_{j=1}^{\infty} \widehat{\theta}\left(N_{j}^{-1} \xi\right) \widehat{\psi_{j}}(\xi)=1, \text { for all } \xi \in \mathbb{R}^{n}
$$

where the functions $\psi_{j} \in \delta$ are defined by

$$
\widehat{\psi_{0}}(\xi)=\frac{\varphi_{0}^{N}(\xi)}{\widehat{\theta_{0}}(\xi)} \quad \text { and } \quad \widehat{\psi_{j}}(\xi)=\frac{\varphi_{j}^{N}(\xi)}{\widehat{\theta}\left(N_{j}^{-1} \xi\right)} \quad \text { for } \quad j \in \mathbb{N} \text {. }
$$

Let us mention that the difference to the classical result is due to the fact that the functions $\psi_{j}$ are in general not obtained simply by dilation from a fixed function $\psi$.

Proof. Recall $\left(\varphi_{j}^{N}\right)_{j \in \mathbb{N}_{0}} \in \Phi^{N}$ is fixed.

Let, as in [FrJa85, Theorem 2.6], $\Theta \in \&$ be a real-valued radial function satisfying

$$
\operatorname{supp} \Theta \subset\left\{x \in \mathbb{R}^{n}:|x| \leqslant 1\right\} \text { and } \widehat{\Theta}(0)=1 .
$$

Then, for some $1>\varepsilon>0$, we have $\widehat{\Theta}(\xi) \geqslant 1 / 2$ for all $\xi$ satisfying $|\xi|<\varepsilon \lambda_{1}$. Then

$$
\theta(x)=\varepsilon^{-n}(-\Delta)^{L} \Theta\left(\frac{x}{\varepsilon}\right)
$$

satisfies requirements (4.73)-(4.77).

Since $\widehat{\theta}\left(N_{j}^{-1} \xi\right) \geqslant c>0$ for $\lambda_{1}^{-1} N_{j} \leqslant|\xi| \leqslant \lambda_{1} N_{j}$, using $\lambda_{1}^{-1} N_{j} \leqslant N_{j-1}$ and $N_{j+1} \leqslant \lambda_{1} N_{j}$ one has $\widehat{\theta}\left(N_{j}^{-1} \xi\right) \geqslant c>0$ for any $\xi \in \operatorname{supp} \varphi_{j}^{N} \subset\left\{\xi \in \mathbb{R}^{n}\right.$ : $\left.N_{j-1} \leqslant|\xi| \leqslant N_{j+1}\right\}$. 
Consequently, the functions $\psi_{j}$ are well defined for $j \geqslant 1$ and

$$
\sum_{j=1}^{\infty} \widehat{\theta}\left(N_{j}^{-1} \xi\right) \widehat{\psi_{j}}(\xi)=1 \quad \text { for any } \xi \in \mathbb{R}^{n} \backslash \operatorname{supp} \varphi_{0}^{N} .
$$

Similarly one has to find $\theta_{0}$ with $\widehat{\theta_{0}}(\xi) \geqslant c>0$ for any $\xi \in \operatorname{supp} \varphi_{0}^{N}$. Taking a function $\Theta \in \delta$ such that $\widehat{\Theta}(\xi) \geqslant 1 / 2$ for $|\xi| \leqslant \delta N_{1}$ the function $\theta_{0}=\delta^{-n} \Theta(x / \delta)$ satisfies the above mentioned requirement. Now one has to define the corresponding function $\psi_{0}$ and the proof is complete.

Remark 4.4.6. From the proof of the above lemma it is clear that for a given system $\left(\varphi_{j}^{N}\right)_{j \in \mathbb{N}_{0}} \in \Phi^{N}$ and fixed functions $\theta_{0}, \theta \in \delta$ the associated system $\left(\psi_{j}\right)_{j \in \mathbb{N}_{0}}$ from (4.78) satisfies

$\widehat{\psi_{j}}(\xi) \geqslant 0$ and $\operatorname{supp} \widehat{\psi_{j}} \subset\left\{\xi \in \mathbb{R}^{n}: N_{j-1} \leqslant|\xi| \leqslant N_{j+1}\right\}$ for any $j \geqslant 1$.

An easy application of Leibniz's rule shows that, for any $\gamma \in \mathbb{N}_{0}^{n}$, there is a constant $c_{\gamma}>0$ (independent of $j$ ) such that

$$
\left|D^{\gamma} \widehat{\psi_{j}}(\xi)\right| \leqslant c_{\gamma}\langle\xi\rangle^{-|\gamma|} \text { for any } \xi \in \mathbb{R}^{n} .
$$

Consequently, each function $\widehat{\psi}_{j}$ is a Fourier multiplier in $L_{p}$, as a simple application of the scalar version of Proposition 3.1.1.

\subsubsection{Proof of the atomic decomposition theorem.}

Part I. Let $g \in F_{p, q}^{\sigma, N}$; we use the method of M. Frazier, B. Jawerth and G. Weiss from [FJW91, Theorem 5.11] to construct atoms and to decompose $g$ as in (4.72).

Let $\theta_{0}, \theta, \psi_{0}$ and $\psi_{v}$ functions in $\$$ satisfying (4.73)-(4.77).

Using $\widehat{\theta}\left(N_{v}^{-1} \xi\right)=N_{v}^{n}\left[\theta\left(N_{v} \cdot\right)\right](\xi)$ we have

$$
g=\theta_{0} * \psi_{0} * g+\sum_{\nu=1}^{\infty} \theta_{N_{\nu}^{-1}} * \psi_{\nu} * g
$$

and using the definition of the cubes $Q_{\nu m}$ we obtain the following equality in $\delta^{\prime}$ :

$$
\begin{aligned}
g(x)= & \sum_{m \in \mathbb{Z}^{n}} \int_{Q_{0 m}} \theta_{0}(x-y)\left(\psi_{0} * g\right)(y) d y \\
& +\sum_{\nu=1}^{\infty} \sum_{m \in \mathbb{Z}^{n}} N_{v}^{n} \int_{Q_{v m}} \theta\left(N_{v}(x-y)\right)\left(\psi_{v} * g\right)(y) d y .
\end{aligned}
$$

We define, for every $v \in \mathbb{N}$ and all $m \in \mathbb{Z}^{n}$,

$$
\lambda_{v m}=C_{\theta} \sigma_{v} N_{v}^{-\frac{n}{p}} \sup _{y \in Q_{v m}}\left|\left(\psi_{v} * g\right)(y)\right|,
$$

where $C_{\theta}=\max \left\{\sup _{|x| \leqslant 1}\left|D^{\alpha} \theta(x)\right|:|\alpha| \leqslant K\right\}$. Define also

$$
\rho_{v m}(x)=\frac{1}{\lambda_{v m}} N_{v}^{n} \int_{Q_{v m}} \theta\left(N_{v}(x-y)\right)\left(\psi_{v} * g\right)(y) d y,
$$

if $\lambda_{v m} \neq 0$ and $\rho_{v m}=0$ otherwise. 
Similarly we define, for every $m \in \mathbb{Z}^{n}$, the numbers $\lambda_{0 m}$ and the functions $\rho_{0 m}$ taking in (4.79) and (4.80) $v=0$ and replacing $\psi_{j}$ and $\theta$ by $\psi_{0}$ and $\theta_{0}$, respectively.

It is obvious that (4.72) is satisfied and it follows by straightforward calculations, using the properties of the functions $\theta_{0}, \theta, \psi_{0}$ and $\psi_{j}$, that $\rho_{0 m}$ are $1_{M}$-N-atoms and that $\rho_{\nu m}$ are $(\sigma, p)_{M, L}-N$-atoms for $v \in \mathbb{N}$.

Finally, we will show that there exists a constant $c>0$ such that $\left\|\lambda \mid f_{p q}^{N}\right\| \leqslant$ $c\left\|g \mid F_{p, q}^{\sigma, N}\right\|$.

We have, for a fixed $v \in \mathbb{N}$,

$$
\begin{aligned}
\sum_{m \in \mathbb{Z}^{n}} \lambda_{v m} \chi_{v m}^{(p)}(x) & =C_{\theta} \sigma_{v} N_{\nu}^{-\frac{n}{p}} \sum_{m \in \mathbb{Z}^{n}} \sup _{y \in Q_{v m}}\left|\left(\psi_{v} * g\right)(y)\right| \cdot N_{\nu}^{\frac{n}{p}} \chi_{v m}(x) \\
& \leqslant c^{\prime} \sigma_{v}\left(\sup _{|z| \leqslant c N_{v}^{-1}} \frac{\left|\left(\psi_{v} * g\right)(x-z)\right|}{\left(1+N_{v}|z|\right)^{r}}\left(1+N_{v}|z|\right)^{r}\right) \\
& \leqslant c^{\prime \prime} \sigma_{v}\left(\psi_{v}^{*} g\right)_{r}(x),
\end{aligned}
$$

since $|x-y| \leqslant c N_{v}^{-1}$ for $x, y \in Q_{v m}$ and $\sum_{m \in \mathbb{Z}^{n}} \chi_{v m}(x)=1$. Here $r>\frac{n}{\min (p, q)}$ and $\left(\psi_{\nu}^{*} g\right)_{r}$ is the maximal function of J. Peetre, compare (4.26). It follows that

$$
\sum_{\nu=1}^{\infty} \sum_{m \in \mathbb{Z}^{n}}\left|\lambda_{\nu m} \chi_{\nu m}^{(p)}(\cdot)\right|^{q} \leqslant c \sum_{\nu=1}^{\infty} \sigma_{\nu}^{q}\left(\psi_{\nu}^{*} g\right)_{r}(\cdot)^{q}
$$

(with the usual modification if $q=\infty$ ) where $c$ is a positive constant.

Now we have to use (4.81) and its counterpart for $v=0$ (which can be obtained by a similar calculation) and get

$$
\left\|\lambda\left|f_{p, q}^{N}\|\leqslant c\|\left(\sum_{\nu=0}^{\infty} \sigma_{\nu}^{q}\left(\psi_{\nu}^{*} g\right)_{r}(\cdot)^{q}\right)^{1 / q}\right| L_{p}\right\| \leqslant c^{\prime}\left\|g \mid F_{p, q}^{\sigma, N}\right\|,
$$

(with the usual modification if $q=\infty$ ) and this completes the proof of the first part of the theorem if we would be able to justify the last inequality in (4.82).

But the last inequality in (4.82) is nothing else than a simple application of Proposition 3.2.1 taking in that theorem $f=\left(f_{v}\right)_{v \in \mathbb{N}_{0}}$, where, for any $v \in \mathbb{N}_{0}$, the function $f_{v}$ is $\sigma_{v}\left(\psi_{v} * g\right)$ and the domain $\Omega_{v}$ is $\left\{\xi \in \mathbb{R}^{n}:|\xi| \leqslant N_{v+1}\right\}$ and recalling the definition of the maximal functions from (4.25) and (4.26).

Part II. Reciprocally, assume now that $g$ can be represented by (4.72), with $M$ and $L$ satisfying (4.69) and (4.70), respectively. We will show that $g \in F_{p, q}^{\sigma, N}$ and that $\left\|g\left|F_{p, q}^{\sigma, N}\|\leqslant c\| \lambda\right| f_{p q}^{N}\right\|$ for some constant $c>0$.

Let $k_{0}$ and $k$ be two functions in $\delta$ such that supp $k_{0}$, supp $k \subset\left\{x \in \mathbb{R}^{n}\right.$ : $|x| \leqslant 1\}$ and $\left|\widehat{k_{0}}(\xi)\right|>0$ for $|\xi| \leqslant N_{1},|\widehat{k}(\xi)|>0$ for $\frac{1}{\lambda_{1}} \leqslant|\xi| \leqslant \lambda_{1}$ and

$$
\int_{\mathbb{R}^{n}} x^{\alpha} k(x) d x=0 \quad \text { for any } \quad|\alpha| \leqslant K .
$$


Our intention is to apply Theorem 4.3.4. Let $K$ be large enough such that $K \geqslant M-1$.

Temporarily let $\nu, j \in \mathbb{N}_{0}, m \in \mathbb{Z}^{n}$ and $x \in \mathbb{R}^{n}$ be fixed; we start finding convenient estimates for $\sigma_{j}\left(k_{N_{j}^{-1}} * \rho_{\nu m}\right)(x)$.

Step II. 1 Let $j \geqslant v$ and let again $k_{N_{j}^{-1}}(x)=N_{j}^{n} k\left(N_{j} x\right)$. Then

$$
\sigma_{j}\left(k_{N_{j}^{-1}} * \rho_{\nu m}\right)(x)=\sigma_{j} \int_{|y| \leqslant 1} k(y) \rho_{\nu m}\left(x-N_{j}^{-1} y\right) d y .
$$

Due to (4.66) the above integral is non-zero only if $\left|x-N_{j}^{-1} y-N_{v}^{-1} m\right| \leqslant c N_{v}^{-1}$ which implies that $x$ is located in some $c Q_{v m}$ since

$$
\left|x-N_{v}^{-1} m\right| \leqslant\left|x-N_{j}^{-1} y-N_{v}^{-1} m\right|+\left|N_{j}^{-1} y\right| \leqslant c N_{v}^{-1}+N_{j}^{-1} \leqslant c^{\prime} N_{v}^{-1} .
$$

According to (4.67) the derivatives $D^{\alpha} \rho_{\nu m}$ exist if $|\alpha| \leqslant M$ so we can use Taylor's expansion theorem of order $M$ for the function $w \mapsto \rho_{\nu m}(w)$ on the set $B\left(x, N_{j}^{-1}\right)$ (the ball centred at $x$ and of radius $N_{j}^{-1}$ ).

Then we put $w=x-N_{j}^{-1} y$ and noting that if $z \in B\left(x, N_{j}^{-1}\right)$ then $z \in c Q_{\nu m}$ we get the expansion

$$
\rho_{\nu m}\left(x-N_{j}^{-1} y\right)=\sum_{|\alpha| \leqslant M-1} c_{\alpha}\left(x-N_{j}^{-1} y-z\right)^{\alpha} D^{\alpha} \rho_{\nu m}(z)+R_{M}(x, y),
$$

where

$$
\begin{aligned}
\left|R_{M}(x, y)\right| & \leqslant c \max _{|\alpha|=M}\left(N_{j}^{-|\alpha|} \sup _{z \in c^{\prime} Q_{v m}}\left|D^{\alpha} \rho_{v m}(z)\right|\right) \\
& \leqslant c^{\prime} N_{j}^{-M} \sigma_{v}^{-1} N_{v}^{\frac{n}{p}+M} \tilde{\chi}_{v m}(x) \\
& =c^{\prime} \sigma_{v}^{-1}\left(N_{j}^{-1} N_{v}\right)^{M} \tilde{\chi}_{v m}^{(p)}(x),
\end{aligned}
$$

for some $c, c^{\prime}>0$, where $\tilde{\chi}_{v m}^{(p)}$ is the $p$-normalised characteristic function of some cube $c Q_{\nu m}$.

Recall that $K$ is large enough such that $K \geqslant M-1$; using the moment conditions for the function $k$ we obtain $\int_{\mathbb{R}^{n}}\left(x-N_{j}^{-1} y-z\right)^{\alpha} k(y) d y=0$ for all $\alpha$ such that $|\alpha| \leqslant M-1$. Hence (4.84) and (4.85) yield

$$
\left|\sigma_{j}\left(k_{N_{j}^{-1}} * \rho_{v m}\right)(x)\right| \leqslant c \sigma_{j} \sigma_{v}^{-1}\left(N_{j}^{-1} N_{v}\right)^{M} \tilde{\chi}_{v m}^{(p)}(x) .
$$

Now using (4.3) and (4.1) we have, for $j \geqslant v$,

$$
\sigma_{j} \sigma_{\nu}^{-1} \leqslant d_{1}^{j-v}=2^{-(j-v)\left(-\log _{2} d_{1}\right)} \quad \text { and } \quad N_{j}^{-1} N_{v} \leqslant \lambda_{0}^{-(j-v)}=2^{-(j-v) \log _{2} \lambda_{0}} .
$$

Inserting the last estimates in (4.86) we get

$$
\left|\sigma_{j}\left(k_{N_{j}^{-1}} * \rho_{\nu m}\right)(x)\right| \leqslant c 2^{-(j-v)\left(-\log _{2} d_{1}+M \log _{2} \lambda_{0}\right)} \widetilde{\chi}_{v m}^{(p)}(x)=2^{-(j-v) \delta} \widetilde{\chi}_{v m}^{(p)}(x),
$$

for $\delta=-\log _{2} d_{1}+M \log _{2} \lambda_{0}$. Clearly $\delta>0$ since $M$ satisfies the estimate (4.69). 
Step II.2 Let now $j<v$. We chose $K$ in (4.83) enough large such that, in addition, $K \geqslant L$. Then

$$
\sigma_{j}\left(k_{N_{j}^{-1}} * \rho_{v m}\right)(x)=\sigma_{j} N_{j}^{n} \int_{\mathbb{R}^{n}} k\left(N_{j} y\right) \rho_{v m}(x-y) d y
$$

and, due to the support localisation of $k$, the above integration can be restricted to the set $\left\{y \in \mathbb{R}^{n}:|y| \leqslant N_{j}^{-1}\right\}$.

We remark also that by our assumption on $j$ and $v$, and to the support localisation for $\rho_{v m}$ one has

$$
\left|x-N_{v}^{-1} m\right| \leqslant\left|x-y-N_{v}^{-1} m\right|+|y| \leqslant c N_{v}^{-1}+N_{j}^{-1} \leqslant c^{\prime} N_{j}^{-1}
$$

and this implies that, if the above integral is non-zero, then $x$ is located in some $c B_{j m}$, where $B_{j m}=\left\{z \in \mathbb{R}^{n}:\left|z-N_{v}^{-1} m\right| \leqslant N_{j}^{-1}\right\}$.

Since $k$ is a smooth function on $\mathbb{R}^{n}$ we may use Taylor's expansion theorem of order $L$ for the function $w \mapsto k(w)$ on the set $B\left(z_{x}, N_{j} N_{v}^{-1}\right)=\left\{w \in \mathbb{R}^{n}\right.$ : $\left.\left|w-z_{x}\right| \leqslant N_{j} N_{v}^{-1}\right\}$, where $z_{x}=z(j, v, m, x)=N_{j}\left(N_{v}^{-1} m-x\right)$.

After that we let $w=N_{j} y$ and get

$$
k\left(N_{j} y\right)=\sum_{|\alpha| \leqslant L} c_{\alpha}\left(N_{j} y-z_{x}\right)^{\alpha} D^{\alpha} k\left(z_{x}\right)+R_{L}(y, x),
$$

where

$$
\left|R_{L}(y, x)\right| \leqslant c\left(N_{j} N_{v}^{-1}\right)^{L+1}
$$

for some positive constant $c$ since $k$ is smooth and has compact support.

By the moment conditions (4.68) we have $\int_{\mathbb{R}^{n}}\left(N_{j} y-z_{x}\right)^{\alpha} \rho_{v m}(x-y) d y=0$ if $|\alpha| \leqslant L$ since we have chosen $K \geqslant L$; using (4.89) we may replace (4.88) by:

$$
\begin{aligned}
\left|\sigma_{j}\left(k_{N_{j}^{-1}} * \rho_{\nu m}\right)(x)\right| & \leqslant \sigma_{j} N_{j}^{n} \int_{|y| \leqslant N_{j}^{-1}}\left|R_{L}(y, x)\right|\left|\rho_{v m}(x-y)\right| d y \\
& \leqslant c \sigma_{j} N_{j}^{n}\left(N_{j} N_{v}^{-1}\right)^{(L+1)} \int_{|y| \leqslant N_{j}^{-1}}\left|\rho_{\nu m}(x-y)\right| d y .
\end{aligned}
$$

Using (4.67) to estimate $\rho_{v m}$ we get

$$
\left|\sigma_{j}\left(k_{N_{j}^{-1}} * \rho_{v m}\right)(x)\right| \leqslant c \sigma_{j} N_{j}^{n}\left(N_{j} N_{v}^{-1}\right)^{(L+1)} \sigma_{v}^{-1} N_{v}^{\frac{n}{p}} \int_{|y| \leqslant N_{j}^{-1}} \tilde{\chi}_{\nu m}(x-y) d y,
$$

where $\tilde{\chi}_{\nu m}$ is the characteristic function of some cube $c Q_{\nu m}$.

Let now $\chi^{j m}$ be the characteristic function of the ball $c B_{j m}$ where $x$ is located; by a straightforward computation we have

$$
\int_{|y| \leqslant N_{j}^{-1}} \tilde{\chi}_{v m}(x-y) d y \leqslant c N_{v}^{-n} \chi^{j m}(x) .
$$


Due to condition (4.70) on $L$ we may choose an $\omega<\min (1, p, q)$ such that

$$
\omega>\frac{n \log _{2} \lambda_{1}}{\log _{2} d_{0}+(L+1+n) \log _{2} \lambda_{0}} .
$$

Denoting by $\mathcal{M} \chi_{v m}$ the Hardy-Littlewood maximal function of $\chi_{v m}$ we get

$$
\chi^{j m}(\cdot) \leqslant c\left(N_{j}^{-1} N_{\nu}\right)^{\frac{n}{\omega}}\left(\mathcal{M} \chi_{\nu m}(\cdot)\right)^{1 / \omega} .
$$

Finally, using (4.91) and (4.93), the estimate (4.90) becomes

$$
\begin{aligned}
& \left|\sigma_{j}\left(k_{N_{j}^{-1}} * \rho_{\nu m}\right)(x)\right| \\
& \leqslant c \sigma_{j} N_{j}^{n}\left(N_{j} N_{v}^{-1}\right)^{(L+1)} \sigma_{v}^{-1} N_{v}^{-n}\left(N_{j}^{-1} N_{v}\right)^{\frac{n}{\omega}}\left(\mathcal{M} \widetilde{\chi}_{v m}^{(p)}(x)\right)^{1 / \omega},
\end{aligned}
$$

where again $\tilde{\chi}_{v m}^{(p)}$ is the $p$-normalised characteristic function of some cube $c Q_{v m}$.

Now using (4.3) and (4.1) we have, for $j<v$,

$$
\begin{gathered}
\sigma_{j} \sigma_{\nu}^{-1} \leqslant d_{0}^{-(\nu-j)}=2^{-(\nu-j)\left(\log _{2} d_{0}\right)} \\
N_{j} N_{v}^{-1} \leqslant \lambda_{0}^{-(\nu-j)}=2^{-(\nu-j) \log _{2} \lambda_{0}} \quad \text { and } \quad N_{j}^{-1} N_{\nu} \leqslant \lambda_{1}^{\nu-j}=2^{-(\nu-j)\left(-\log _{2} \lambda_{1}\right)}
\end{gathered}
$$

so that (4.94) becomes

$$
\begin{aligned}
& \left|\sigma_{j}\left(k_{N_{j}^{-1}} * \rho_{v m}\right)(x)\right| \\
\leqslant & c 2^{-(\nu-j) \log _{2} d_{0}} 2^{-(\nu-j)(L+1+n) \log _{2} \lambda_{0}} 2^{-(v-j) \frac{n}{\omega}\left(-\log _{2} \lambda_{1}\right)}\left(\mathcal{M} \widetilde{\chi}_{v m}^{(p)}(x)\right)^{1 / \omega} \\
= & c 2^{-(v-j) \varepsilon}\left(\mathcal{M} \widetilde{\chi}_{v m}^{(p)}(x)\right)^{1 / \omega},
\end{aligned}
$$

where

$$
\varepsilon=\log _{2} d_{0}+(L+1+n) \log _{2} \lambda_{0}-\frac{n}{\omega} \log _{2} \lambda_{1}>0,
$$

due to our choice of $\omega$, see (4.92).

Remark that the terms with $j=0$ and/or $v=0$ can also be covered by the technique in Steps II.1-2.

Step II.3 Using (4.87) and (4.95) we get, for $0<q \leqslant 1$,

$$
\begin{gathered}
\left|\sigma_{j}\left(k_{N_{j}^{-1}} * \sum_{\nu=0}^{\infty} \sum_{m \in \mathbb{Z}^{n}} \lambda_{\nu m} \rho_{\nu m}\right)(x)\right|^{q} \leqslant c \sum_{\nu \leqslant j} \sum_{m \in \mathbb{Z}^{n}}\left|\lambda_{\nu m}\right|^{q} 2^{-\delta(j-v) q} \widetilde{\chi}_{\nu m}^{(p) q}(x)+ \\
+c^{\prime} \sum_{\nu>j} \sum_{m \in \mathbb{Z}^{n}}\left|\lambda_{v m}\right|^{q} 2^{-\varepsilon(\nu-j) q}\left(\mathcal{M} \tilde{\chi}_{v m}^{(p)}(x)\right)^{q / \omega}
\end{gathered}
$$

with $\delta, \varepsilon>0$, with the usual modification if $1<q \leqslant \infty$. that

We sum over $j$, take the $\frac{1}{q}$-th power and then the $L_{p}$-quasi-norm and obtain

$$
\left\|\left(\sum_{j=1}^{\infty} \sigma_{j}^{q}\left|\left(k_{N_{j}^{-1}} * \sum_{\nu=0}^{\infty} \sum_{m \in \mathbb{Z}^{n}} \lambda_{\nu m} \rho_{\nu m}\right)(\cdot)\right|^{q}\right)^{1 / q} \mid L_{p}\right\|
$$


can be estimated from above by

$$
\begin{aligned}
& c\left\|\left(\sum_{\nu=0}^{\infty} \sum_{m \in \mathbb{Z}^{n}}\left|\lambda_{v m}\right|^{q} \tilde{\chi}_{v m}^{(p)}(\cdot)^{q}\right)^{1 / q} \mid L_{p}\right\| \\
+ & c^{\prime}\left\|\left(\sum_{\nu=0}^{\infty} \sum_{m \in \mathbb{Z}^{n}}\left|\lambda_{v m}\right|^{q}\left(\mathcal{M} \tilde{\chi}_{\nu m}^{(p)}(\cdot)\right)^{q / \omega}\right)^{1 / q} \mid L_{p}\right\|,
\end{aligned}
$$

with the usual modification if $q=\infty$.

The first term of (4.96) is just what we want since $\widetilde{\chi}_{v m}^{(p)}$ can be replaced by $\chi_{v m}^{(p)}$. With $h_{v m}=\lambda_{v m} \chi_{v m}^{(p)}$ the second term of (4.96) can be written as

$$
c^{\prime \prime}\left\|\left(\sum_{\nu=0}^{\infty} \sum_{m \in \mathbb{Z}^{n}} \mathcal{M} h_{v m}^{\omega}(\cdot)^{q / \omega}\right)^{\omega / q} \mid L_{p / \omega}\right\|^{\frac{1}{\omega}}
$$

(usual modification if $q=\infty$ ). Recall that $1<\frac{p}{\omega}<\infty$ and $1<\frac{q}{\omega} \leqslant \infty$ so that we can apply the Fefferman-Stein inequality and again obtain what we want.

The term with $j=0$ can be incorporated by the same technique.

\subsubsection{Comments}

Remark 4.4.7. Let $d>0$ be given, let $v \in \mathbb{N}_{0}$ and $m \in \mathbb{Z}^{n}$ fixed and let us denote by $R_{v m}$ a cube with sides parallel to the axes, centred at $x^{v m}$ where

$$
\left|x^{v m}-N_{v}^{-1} m\right| \leqslant d N_{v}^{-1}
$$

and with side length $N_{v}^{-1}$.

Then let $c>0$ be chosen in dependence of $d$ such that, for every choice of $v \in \mathbb{N}_{0}$ and all choices of $x^{v m}$ in (4.97), we have

$$
\bigcup_{m \in \mathbb{Z}^{n}} c R_{v m}=\mathbb{R}^{n}
$$

It is clear from the previous proof that we may replace in Definition 4.4.1 the cube $Q_{v m}$ by $R_{v m}$, with the number $c$ being from (4.98).

A similar remark in the classical case $\left(N_{v}=2^{v}\right.$ and $\left.\sigma_{v}=2^{v s}, s \in \mathbb{R}\right)$ turned out to be very useful in the work of H. Triebel and H. Winkelvoß, [TrWi96].

Remark 4.4.8. Let $\sigma=\left(\sigma_{j}\right)_{j \in \mathbb{N}_{0}}$ be an admissible sequence. A lower, respectively a upper, index of the sequence $\sigma$, was introduced in [Br02] by

$$
\underline{\mathfrak{s}}(\sigma)=\liminf _{j \rightarrow \infty} \log \left(\frac{\sigma_{j+1}}{\sigma_{j}}\right) \quad \text { and } \quad \overline{\mathfrak{s}}(\sigma)=\limsup _{j \rightarrow \infty} \log \left(\frac{\sigma_{j+1}}{\sigma_{j}}\right) ;
$$

clearly, based on (4.3), the above numbers are finite and they satisfy $\underline{\mathfrak{s}}(\sigma) \leqslant$ $\overline{\mathfrak{s}}(\sigma)$. Moreover, for any $d_{0}^{*}<2^{\mathfrak{s}(\sigma)}$ and for any $d_{1}^{*}>2^{\overline{\mathfrak{s}}(\sigma)}$ there exists a natural number $J=J\left(d_{0}^{*}, d_{1}^{*}\right)$ such that $d_{0}^{*} \sigma_{j} \leqslant \sigma_{j+1} \leqslant d_{1}^{*} \sigma_{j}$, for any $j \geqslant J$. Similar considerations hold true (with obvious appropriate changes) for an admissible sequence $N=\left(N_{j}\right)_{j \in \mathbb{N}_{0}}$.

However a change of the sequences $\sigma$ and $N$ in the first $J$ terms will not change the spaces $B_{p, q}^{\sigma, N}$, respectively $F_{p, q}^{\sigma, N}$, up to equivalent quasi-norms. 
Based on the above remark and following the proofs in the previous sections, it is clear that we may replace in the statements of the main results (local means and atomic decomposition theorem) the numbers $d_{0}$ and $d_{1}$ by $2 \mathfrak{\underline { \mathfrak { s } }}(\sigma)$, respectively $2^{\overline{\mathfrak{s}}(\sigma)}$, and the numbers $\lambda_{0}$ and $\lambda_{1}$ by $2^{\mathfrak{s}(N)}$, respectively $2^{\overline{\mathfrak{s}}(N)}$.

More precisely, the assumption $\lambda_{0}>1$ in (4.1) has to be replaced by $\underline{\mathfrak{s}}(N)>0$ and in Theorem 4.3.4 (local means) condition (4.27) can be replaced by

$$
K>-1+\frac{\overline{\mathfrak{s}}(\sigma)}{\underline{\mathfrak{s}}(N)} .
$$

Moreover, in Theorem 4.4.3 (atomic decomposition) one can replace condition (4.69) by

$$
M>\frac{\overline{\mathfrak{s}}(\sigma)}{\underline{\mathfrak{s}}(N)},
$$

and condition (4.70), respectively (4.71), by

$$
L>-1+n\left(\frac{\overline{\mathfrak{s}}(N)}{\underline{\mathfrak{s}}(N)} \frac{1}{\min (1, p, q)}-1\right)-\frac{\underline{\mathfrak{s}}(\sigma)}{\underline{\mathfrak{s}}(N)},
$$

respectively

$$
L>-1+n\left(\frac{\overline{\mathfrak{s}}(N)}{\underline{\mathfrak{s}}(N)} \frac{1}{\min (1, p)}-1\right)-\frac{\underline{\mathfrak{s}}(\sigma)}{\underline{\mathfrak{s}}(N)} .
$$

\subsection{Examples}

For people interested in concrete situations we would like to point out in this section how our main results (the theorem on local means and the atomic decomposition theorem) look in some special cases.

4.5.1. The classical case. As we have already mentioned several times in this work, if $N_{j}=2^{j}$, and $\sigma_{j}=2^{j s}, s \in \mathbb{R}$, then the spaces $B_{p, q}^{\sigma, N}$ and $F_{p, q}^{\sigma, N}$ are the classical spaces $B_{p, q}^{s}$ and $F_{p, q}^{s}$.

Condition (4.1) is fulfilled with $\lambda_{0}=\lambda_{1}=2$. Moreover, condition (4.3) is fulfilled with $d_{0}=d_{1}=2^{s}$.

The restriction (4.27) in the theorem on local means is then $K>-1+s$. Note that if $s<0$ there are no moment conditions needed. Theorem 4.3 .4 coincides with the result of H.-Q. Bui, M. Paluszyński and M. Taibleson as it was already mentioned.

The restrictions (4.69), (4.70), respectively (4.71), and their counterparts, in the atomic decomposition theorem are $M>s$ and

$$
L>-1+n\left(\frac{1}{\min (1, p, q)}-1\right)-s,
$$

respectively

$$
L>-1+n\left(\frac{1}{\min (1, p)}-1\right)-s,
$$

which are essentially the restrictions from the works of M. Frazier and B. Jawerth, cf. also the formulation in [Tr97]. 
4.5.2. The spaces $B_{p, q}^{(s, \Psi)}$ and $F_{p, q}^{(s, \Psi)}$. In Example 2.2 .5 we mentioned that if $s \in \mathbb{R}$ is fixed and

$$
\sigma_{j}=2^{j s} \Psi\left(2^{-j}\right), \quad j \in \mathbb{N}_{0}
$$

where $\Psi$ is a positive monotone function on $(0,1]$ such that there are positive constants $b_{0}$ and $b_{1}$ with

$$
b_{0} \Psi\left(2^{-j}\right) \leqslant \Psi\left(2^{-2 j}\right) \leqslant b_{1} \Psi\left(2^{-j}\right) \quad \text { for all } j \in \mathbb{N}_{0},
$$

then $\sigma$ is an admissible sequence with appropriate $d_{0}=b_{0}^{\prime} 2^{s}$ and $d_{1}=b_{1}^{\prime} 2^{s}$ in (2.2).

If, in addition $N_{j}=2^{j}$ then the spaces $B_{p, q}^{\sigma, N}$ and $F_{p, q}^{\sigma, N}$ are the spaces $B_{p, q}^{(s, \Psi)}$ and $F_{p, q}^{(s, \Psi)}$ considered in [Mo99] and [Mo01].

Condition (4.1) is fulfilled with $\lambda_{0}=\lambda_{1}=2$.

The restriction (4.27) in the theorem on local means is then $K>-1+s+$ $\log _{2} b_{1}$.

The restrictions (4.69), (4.70), respectively (4.71), in the atomic decomposition theorem are $M>s+\log _{2} b_{1}^{\prime}$ and

$$
L>-1+n\left(\frac{1}{\min (1, p, q)}-1\right)-s-\log _{2} b_{0}^{\prime},
$$

respectively

$$
L>-1+n\left(\frac{1}{\min (1, p)}-1\right)-s-\log _{2} b_{0}^{\prime} .
$$

Our conditions concerning the constants $K, L$ and $M$ are slightly different (because of the additional log-terms) from those in the atomic decomposition of S. Moura, see [Mo01, Theorem 1.18], which was proved directly for the spaces $B_{p, q}^{(s, \Psi)}$ and $F_{p, q}^{(s, \Psi)}$ themselves.

4.5.3. Function spaces associated to a continuous negative definite function. We have already mentioned in the Introduction as well as in Section 3.3 that in [FJS01a] and [FJS01b] Bessel potential spaces $H_{p}^{\psi, s}\left(\mathbb{R}^{n}\right)$ associated to a continuous negative definite function $\psi: \mathbb{R}^{n} \rightarrow \mathbb{R}$ were introduced and studied in the context of constructing a Markov process starting in every point of $\mathbb{R}^{n}$.

Recall (3.19) for the norm in $H_{p}^{\psi, s}(1<p<\infty, s \in \mathbb{R})$.

In Section 3.3 we have noticed that considering the continuous negative definite function $\psi: \mathbb{R}^{n} \rightarrow \mathbb{R}$ of the form $\psi(\xi)=f\left(1+|\xi|^{2}\right), \xi \in \mathbb{R}^{n}$, where $f$ is a Bernstein function with $\lim _{t \rightarrow \infty} f(t)=\infty$, then the spaces $H_{p}^{\psi, s}\left(\mathbb{R}^{n}\right)$ can be regarded as function spaces of generalised smoothness.

More precisely, if $\psi(\xi)=f\left(1+|\xi|^{2}\right)$ then Corollary 3.1.21 leads to

$$
H_{p}^{f\left(1+|\cdot|^{2}\right), s}=F_{p, 2}^{\sigma^{s}, N^{\psi, 2}}
$$

where, as usual, $\sigma^{s}=\left(2^{j s}\right)_{j \in \mathbb{N}_{0}}$ and the sequence $N^{\psi, 2}=\left(N_{j}^{\psi, 2}\right)_{j \in \mathbb{N}_{0}}$ is determined, cf. (3.17), as follows

$$
N_{j}^{\psi, 2}=\sup \left\{|\xi|: f\left(1+|\xi|^{2}\right) \leqslant 2^{2 j}\right\} \quad \text { for any } j \in \mathbb{N}_{0}
$$


For simplicity let $N_{j}=N_{j}^{\psi, 2}$. Obviously $\left(N_{j}\right)_{j \in \mathbb{N}_{0}}$ is increasing. Clearly, for any $j$, there exists a $\xi^{(j)}$ such that $\left|\xi^{(j)}\right|=N_{j}$ and consequently $f\left(1+N_{j}^{2}\right)=$ $f\left(1+\left|\xi^{(j)}\right|^{2}\right)=2^{2 j}$.

- Each Bernstein function satisfies $f(c t) \leqslant c f(t)$ for any $c \geqslant 1$ (see for example [Ja01, Lemma 3.9.34]) so that $f\left(4 \cdot\left(1+N_{j}^{2}\right)\right) \leqslant 4 \cdot f\left(1+N_{j}^{2}\right)=2^{2(j+1)}$ which immediately implies $2 N_{j} \leqslant N_{j+1}$. Consequently $N=\left(N_{j}\right)_{j \in \mathbb{N}_{0}}$ is strongly increasing and satisfies the first inequality in (4.1) with $\lambda_{0}=2$.

- Unfortunately, for general Bernstein functions $f$ with $\lim _{t \rightarrow \infty} f(t)=\infty$ the inequality $N_{j+1} \leqslant \lambda_{1} N_{j}$ is not always satisfied.

However, if the Bernstein function $f$ with $\lim _{t \rightarrow \infty} f(t)=\infty$ additionally satisfies there is an $\quad r \in(0,1] \quad$ such that $\quad t \mapsto f_{(r)}(t)=\frac{f(t)}{t^{r}} \quad$ is increasing,

then $N_{j+1} \leqslant \lambda_{1} N_{j}$ for some $\lambda_{1}>1$, at least if $\lambda_{1}$ satisfies $\lambda_{1}^{2} \geqslant 2^{2 / r}+\left(2^{2 / r}-\right.$ 1) $N_{0}^{-2}$. Indeed, from $f_{(r)}\left(1+N_{j}^{2}\right) \leqslant f_{(r)}\left(1+N_{j+1}^{2}\right)$ we get $N_{j+1}^{2} \leqslant 2^{2 / r} N_{j}^{2}+$ $2^{2 / r}-1 \leqslant \lambda_{1}^{2} N_{j}^{2}$ for any such $\lambda_{1}$.

Note that in (4.104) one cannot assume $r=0$. One should also note that the function $f(t)=\log (1+t)$ does not satisfy condition (4.104) whereas the functions $\sqrt{t} \log (1+\sqrt{t})$ and $\sqrt{t}(1-\exp (-4 \sqrt{t}))$ satisfy condition (4.104) with $r=1 / 2$.

One should also note that (4.104) is in some sense not surprising since it implies $f(t) \geqslant c t^{r}$ for large $t$ and this leads to $\psi(\xi)=f\left(1+|\xi|^{2}\right) \geqslant C\left(1+|\xi|^{2}\right)^{r}$ if $\xi \in \mathbb{R}^{n}$.

When treating continuous negative definite functions, the last inequality is a restriction often used, see, for example, [Ja01] and the references therein.

Consequently, if $f$ is a Bernstein function with $\lim _{t \rightarrow \infty} f(t)=\infty$ which satisfies (4.104) condition (4.1) is fulfilled with $\lambda_{0}=2$ and with some $\lambda_{1}$ with $\lambda_{1} \geqslant$ $2^{1 / r} \geqslant 2$.

Clearly condition (4.3) is fulfilled with $d_{0}=d_{1}=2^{s}$.

The restriction (4.27) in the theorem on local means is then $K>-1+s$.

The restrictions (4.69) and (4.70) in the atomic decomposition theorem are $M>s$ and

$$
L>-1+n\left(\frac{\log _{2} \lambda_{1}}{\min (1, p, 2)}-1\right)-s=-1+n\left(\log _{2} \lambda_{1}-1\right)-s .
$$

\section{References}

[AdHe96] Adams, D.R., Hedberg, L.I.: Function spaces and potential theory. Berlin: Springer 1996

[BPT96] Bui, H.-Q., Paluszyński, M., Taibleson, M.: A maximal characterization of weighted Besov-Lipschitz and Triebel-Lizorkin spaces. Stud. Math. 119, 219246 (1996)

[BPT97] Bui, H.-Q., Paluszyński, M., Taibleson, M.: Characterization of the BesovLipschitz and Triebel-Lizorkin spaces. The case $q<1$. J. Fourier Anal. Appl. 3, 837-846 (1997) 
[Bu99] Burenkov, V.: Extension theorems for Sobolev spaces. Oper. Theory Adv. Appl. 109, 187-200 (1999)

[Br02] Bricchi, M.: Tailored function spaces and related $h$-sets. PhD Thesis. University of Jena 2002

[CoFe86] Cobos, F., Fernandez, D.L.: Hardy-Sobolev spaces and Besov spaces with a function parameter. Proc. Lund Conf. 1986, Lect. Notes Math. 1302, 158-170. Berlin: Springer 1986

[Co66] Courrège, Ph.: Sur la forme intégro-différentielle des opérateurs de $C_{K}^{\infty}$ dans $\mathbb{C}$ satisfaisant au principe du maximum. Exposé 2. Sém. Théorie du Potentiel, 1965/66, $38 \mathrm{pp}$

[EdHa99] Edmunds, D.E., Haroske, D.: Spaces of Lipschitz type, embeddings and entropy numbers. Diss. Math. 380, 1-43 (1999)

[EGO97] Edmunds, D.E., Gurka, P., Opic, B.: On embeddings of logarithmic Bessel potential spaces. J. Funct. Anal. 146, 116-150 (1997)

[EdTr96] Edmunds, D.E., Triebel, H.: Function spaces, entropy numbers, differential operators. Cambridge: Cambridge University Press 1996

[EdTr98] Edmunds, D.E., Triebel, H.: Spectral theory for isotropic fractal drums. C. R. Acad. Sci. Paris 326, 1269-1274 (1998)

[EdTr99] Edmunds, D.E., Triebel, H.: Eigenfrequencies of isotropic fractal drums. Oper. Theory Adv. Appl. 110, 81-102 (1999)

[FJS01a] Farkas, W., Jacob, N., Schilling, R.L.: Feller semigroups, $L^{p}$-sub-Markovian semigroups, and applications to pseudo-differential operators with negative definite symbols. Forum Math. 13, 59-90 (2001)

[FJS01b] Farkas, W., Jacob, N., Schilling, R.L.: Function spaces related to continuous negative definite functions: $\psi$-Bessel potential spaces. Diss. Math. 393, 1-62 (2001)

[FaLe01] Farkas, W., Leopold, H.G.: Characterisations of function spaces of generalised smoothness. Jenaer Schriften zur Mathematik und Informatik 23, 1-56 (2001)

[FeSt71] Fefferman, C., Stein, E.M.: Some maximal inequalities. Am. J. Math. 93, 107$115(1971)$

[FrJa85] Frazier, M., Jawerth, B.: Decomposition of Besov spaces. Indiana Univ. Math. J. 34, 777-799 (1985)

[FrJa90] Frazier, M., Jawerth, B.: A discrete transform and decomposition of distribution spaces. J. Funct. Anal. 93, 34-170 (1990)

[FJW91] Frazier, M., Jawerth, B.: Littlewood-Paley theory and the study of function spaces. CBMS Reg. Conf. Ser. Math. 79. Providence, RI: Am. Math. Soc. 1991

[Fu71] Fukushima, M.: Dirichlet spaces and strong Markov processes. Trans. Am. Math. Soc. 162, 185-224 (1971)

[Go76] Goldman, M.L.: A description of the trace space for functions of a generalized Hölder class. Dokl. Akad. Nauk SSSR 231, 525-528 (1976)

[Go79] Goldman, M.L.: A description of the traces of some function spaces Trudy Mat. Inst. Steklov 150, 99-127 (1979). English transl.: Proc. Steklov Inst. Math. 1981, no. 4 (150)

[Go80] Goldman, M.L.: A method of coverings for describing general spaces of Besov type. Trudy Mat. Inst. Steklov 156, 47-81 (1980). English transl.: Proc. Steklov Inst. Math. 1983, no. 2 (156)

[Go84a] Goldman, M.L.: Imbedding theorems for anisotropic Nikol'skii-Besov spaces with moduli of continuity of general type. Trudy Mat. Inst. Steklov 170, 86-104 (1984). English transl.: Proc. Steklov Inst. Math. 1987, no. 1 (170)

[Go84b] Goldman, M.L.: Embedding of Nikol'skii-Besov spaces with moduli of continuity of general type in Lorentz spaces. Dokl. Akad. Nauk SSSR 277, 20-24 (1984)

[Go85] Goldman, M.L.: On imbedding generalized Nikol'skii-Besov spaces in Lorentz spaces. Trudy Mat. Inst. Steklov 172, 128-139 (1985). English transl.: Proc. Steklov Inst. Math. 1987, no. 3 (172) 
[Go86] Goldman, M.L.: Embedding constructive and structural Lipschitz spaces in symmetric spaces. Trudy Mat. Inst. Steklov 173, 90-112 (1986). English transl.: Proc. Steklov Inst. Math. 1987, no. 4 (173)

[Go89] Goldman, M.L.: Traces of functions with restrictions on the spectrum. Trudy Mat. Inst. Steklov 187, 69-77 (1989). English transl.: Proc. Steklov Inst. Math. 1990, no. 3 (187)

[Go92] Goldman, M.L.: A criterion for the embedding of different metrics for isotropic Besov spaces with arbitrary moduli of continuity. Trudy Mat. Inst. Steklov 201, 186-218 (1992). English transl.: Proc. Steklov Inst. Math. 1994, no. 2 (201)

[Ja96] Jacob, N.: Pseudo-differential operators and Markov processes. Berlin: Akademie Verlag 1996

[Ja98] Jacob, N.: Characteristic functions and symbols in the theory of Feller processes. Potential Analysis 8, 61-68 (1998)

[Ja01] Jacob, N.: Pseudo-differential operators and Markov processes. Vol. 1: Fourier analysis and semigroups, World Scientific, Imperial College Press 2001

[JaSc96] Jacob, N., Schilling, R.L.: Subordination in the sense of Bochner - An approach through pseudo-differential operators. Math. Nachr. 178, 199-231 (1996)

[Ka77a] Kalyabin, G.A.: Characterization of spaces of generalized Liouville differentiation. Mat. Sb. Nov. Ser. 104, 42-48 (1977)

[Ka77b] Kalyabin, G.A.: Imbedding theorems for generalized Besov and Liouville spaces. Dokl. Akad. Nauk SSSR 232, 1245-1248 (1977). English transl.: Soviet Math. Dokl. 1977, no 1 (18)

[Ka78] Kalyabin, G.A.: Trace spaces for generalized anisotropic Liouville classes. Izv. Akad. Nauk SSSR Ser. Mat. 42, 305-314 (1978). English transl.: Math. USSR Izv. 12 (1978)

[Ka79] Kalyabin, G.A.: A description of traces for anisotropic spaces of in classes of Triebel-Lizorkin type. Trudy Mat. Inst. Steklov 150, 160-173 (1979). English transl.: Proc. Steklov Inst. Math. 1981, no. 4 (150)

[Ka80] Kalyabin, G.A.: Description of functions in classes of Besov-Lizorkin-Triebel type. Trudy Mat. Inst. Steklov 156, 82-109 (1980). English transl.: Proc. Steklov Institut Math. 1983, no. 2 (156)

[Ka81] Kalyabin, G.A.: Criteria for multiplicativity and imbedding in $C$ for spaces of Besov-Lizorkin-Triebel type. Mat. Zametki 30, 517-526 (1981). English transl.: in Math. Notes 1983, no. 30

[Ka88] Kalyabin, G.A.: Characterization of spaces of Besov-Lizorkin-Triebel type by means of generalized differences. Trudy Mat. Inst. Steklov 181, 95-116 (1988). English transl.: Proc. Steklov Inst. Math. 1989, no. 4 (181)

[KaLi87] Kalyabin, G.A., Lizorkin, P.I.: Spaces of functions of generalized smoothness. Math. Nachr. 133, 7-32 (1987)

[KuNi88] Kudryavtsev, L.D., Nikol'skiǐ, S.M.: Spaces of differentiable functions of several variables and imbedding theorems. Analysis III, Spaces of differentiable functions, Encyclopadia of Math. Sciences 26, 4-140. Heidelberg: Springer 1990

[Le90] Leopold, H.G.: Spaces of variable and generalised smoothness. Manuscript, Jena, 1990

[Le98] Leopold, H.G.: Embeddings and entropy numbers in Besov spaces of generalized smoothness. in: Function Spaces: The fifth conference, Lecture Notes Pure Appl. Math. 213, 323-336. Hudzik, H., Skrzypczak, L.(eds.). Marcel Dekker 2000

[Me83] Merucci, C.: Applications of interpolation with a function parameter to Lorentz, Sobolev and Besov spaces. Proc. Lund Conf. 1983. Lect. Notes Math. 1070, 183-201. Berlin: Springer

[Mo99] Moura, S.: Some properties of the spaces $F_{p q}^{(s, \Psi)}\left(\mathbb{R}^{n}\right)$ and $B_{p q}^{(s, \Psi)}\left(\mathbb{R}^{n}\right)$. Preprint 99-09, Univ. Coimbra 1999

[Mo01] Moura, S.: Function spaces of generalised smoothness. Diss. Math. 398, 1-87 (2001) 
[Ne88] Netrusov, Y.V.: Embedding theorems for traces of Besov spaces and LizorkinTriebel spaces. Dokl. Akad. Nauk SSSR 298, 1326-1330 (1988). English transl.: Soviet Math. Dokl. 1988, no. 1 (37)

[Ne89] Netrusov, Y.V.: Metric estimates of the capacities of sets in Besov spaces. Trudy Mat. Inst. Steklov 190, 159-185 (1989). English transl.: Proc. Steklov Inst. Math. 1992, no. 1 (190)

[OpTr00] Opic, B., Trebels, W.: Bessel potentials with logarithmic components and Sobolev-type embeddings. Anal. Math. 26, 299-319 (2000)

[Pe75] Peetre, J.: On spaces of Triebel Lizorkin type. Ark. Mat. 13, 123-130 (1975)

[Ry99] Rychkov, V.: On a theorem of Bui, Paluszyński, and Taibleson. Trudy Mat. Inst. Steklov 227, 286-298 (1999). English transl.: Proc. Steklov Inst. Math. 227, 280-292 (1999)

[Sc94] Schilling, R.L.: Zum Pfadverhalten von Markovschen Prozessen, die mit Lévy Prozessen vergleichbar sind. Dissertation Universität Erlangen-Nürnberg, Erlangen 1994

[Sc98a] Schilling, R.L.: Subordination in the sense of Bochner and a related functional calculus. J. Aust. Math. Soc. 64, 368-396 (1998)

[Sc98b] Schilling, R.L.: Conservativeness of semigroups generated by pseudodifferential operators. Potential Anal. 9, 91-104 (1998)

[ScTr87] Schmeisser, H.J., Triebel, H.: Topics in Fourier analysis and function spaces. Leipzig: Geest \& Portig 1987

[StWe71] Stein, E.M., Weiss, G.: Introduction to Fourier analysis on euclidean spaces. Princeton, NJ: Princeton Univ. Press 1971

[StTo89] Strömberg, J.O., Torchinsky, A.: Weighted Hardy spaces. Lect. Notes Math. 1381. Berlin: Springer 1989

[Tr77] Triebel, H.: Fourier analysis and function spaces. Teubner-Texte Math. 7. Leipzig: Teubner 1977

[Tr78] Triebel, H.: Interpolation theory, function spaces, differential operators. Amsterdam: North Holland 1978

[Tr83] Triebel, H.: Theory of function spaces. Leipzig: Geest \& Portig and Basel: Birkhäuser 1983

[Tr86] Triebel, H.: Theory of function spaces. Russian ed. Moscow: Mir 1986

[Tr88] Triebel, H.: Characterizations of Besov-Hardy-Sobolev spaces: a unified approach. J. Approximation Theory 52, 162-203 (1988)

[Tr92] Triebel, H.: Theory of function spaces II. Basel: Birkhäuser 1992

[Tr97] Triebel, H.: Fractals and spectra, related to Fourier Analysis and Function Spaces. Basel: Birkhäuser 1997

[Tr01] Triebel, H.: The structure of functions. Basel: Birkhäuser 2001

[TrWi96] Triebel, H., Winkelvoß, H.: A Fourier analytical characterization of the Hausdorff dimension of a closed set and of related Lebesgue spaces. Stud. Math. 121, 149-166 (1996) 